\title{
The Structure of Titan's Stratosphere from the 28 Sgr Occultation
}

\author{
B. Sicardy ${ }^{1}$ \\ Observatoire de Paris, DESPA, Paris 6 Université, 92195 Meudon Cédex Principal, France \\ E-mail: sicardy@mesiob.obspm.fr \\ F. Ferri \\ CISAS “G. Colombo,” Università di Padova, Via Venezia, 1, I-35131 Padova, Italy \\ F. Roques, J. Lecacheux, and S. Pau \\ Observatoire de Paris, DESPA, 92195 Meudon Cédex Principal, France \\ N. Brosch \\ Department of Astronomy and Astrophysics and the Wise Observatory, Beverly and Raymond Sackler Faculty of Exact Sciences, \\ Tel Aviv University, Tel Aviv 69978, Israel \\ Y. Nevo \\ Kibbutz Ein Harod Meuchad, 18965, Israel \\ W. B. Hubbard \\ Lunar and Planetary Laboratory, University of Arizona, Tucson, Arizona 85721
}

H. J. Reitsema

Ball Aerospace Systems Division, Boulder, Colorado 80306

C. Blanco

Istituto di Astronomia, Universita di Catania, Via Andrea Doria, 6, I-95125 Catania, Italy

E. Carreira

Specola Vaticana, Vatican Observatory Research Group, V-00120 Città del Vaticano

W. Beisker, C. Bittner, H.-J. Bode, M. Bruns, H. Denzau, M. Nezel, E. Riedel, and H. Struckmann

IOTA-European Section, Bartold-Knaust Strasse 8, 3000 Hannover 91, Germany

G. Appleby

Royal Greenwich Observatory, Madingley Road, Cambridge, CB4 4QR, United Kingdom

R. W. Forrest and I. K. M. Nicolson

University of Hertfordshire Observatory, Bayfordbury, Hertford, SG13 8LD, United Kingdom

and

A. J. Hollis and R. Miles

Asteroids and Remote Planets Section, British Astronomical Society, Burlington House, Piccadilly, London WIV 9AG, United Kingdom

Received February 25, 1998; revised July 12, 1999

\footnotetext{
A dozen lightcurves obtained during the ground-based observations of the occultation of 28 Sgr by Titan (3 J uly 1989) are reanalyzed. Profiles of density and temperature between altitude levels $z$

${ }^{1}$ Partly supported by a grant from the Institut Universitaire de France.
}

of 290 and $500 \mathrm{~km}$ (pressures $p$ from 110 to $1.4 \mu \mathrm{bar}$ ) are derived. A mean number-density scale height of $50.5 \pm 1.4 \mathrm{~km}$ is found with no significant difference between immersion and emersion. Two inversion layers are observed at 425 and $\mathbf{4 5 0}-\mathbf{4 5 5} \mathrm{km}$, respectively ( $p \sim 7 \mu$ bar and $p \sim 4 \mu$ bar), with an increase in temperature of about $10 \mathrm{~K}$ in less than $\Delta z=10 \mathrm{~km}$. These layers are visible both 
at immersion and at emersion, at latitudes ranging from $46^{\circ} S$ to $20^{\circ} \mathrm{N}$, and are thus global features of the stratosphere. The profiles of temperature gradients exhibit a clear cutoff at the adiabatic lapse rate, indicating that fluctuations lead to marginal convective instabilities. Although ray crossing can also cause an apparent cutoff of the temperature gradients, we estimate it probably does not play an important role in the observed cutoff, at least for the larger structures under study. The vertical power spectra of fluctuations show a general power law behavior, with an exponent close to -3 , between vertical wavelengths of $\sim 5$ and $50 \mathrm{~km}$. The finite stellar diameter and ray crossings can distort the real spectra, and we can only conclude that the original power spectra have slopes between -2 and -3 . The horizontal structure of the atmosphere exhibits typical aspect (horizontal-to-vertical) ratios of 15-45, with a tail in the distribution with values as high as $100-200$ for some structures. Finally, the horizontal spectrum of fluctuations is a power law with an exponent close to $\mathbf{- 4}$ (between horizontal wavelengths of $\sim 25$ and $250 \mathrm{~km}$ ), if we assume it is separable from the vertical spectrum. (c) 1999 A cademic Press

Key Words: Titan; atmospheres (dynamics and structure); occultations.

\section{INTRODUCTION}

On July 3rd, 1989, the bright star 28 Sagittarii (28 Sgr, $V \sim 5.5)$ was occulted by Saturn, its rings, and a few hours later, Titan's thick atmosphere. This exceptional event was followed from Europe, the Middle East and Central Asia by professional and amateur astronomers, at wavelengths ranging from 0.36 to $0.89 \mu \mathrm{m}$, with telescopes between $15 \mathrm{~cm}$ and $2 \mathrm{~m}$ in diameter.

The occultation lightcurves provide information on Titan's atmosphere at altitude levels between about 250 and $600 \mathrm{~km}$ above the satellite's surface. This corresponds to pressure levels of about 250 and $0.15 \mu$ bar, respectively. The atmospheric layers below $250 \mathrm{~km}$ are inaccessible because the stellar flux is then too much refracted and also because Titan's atmosphere becomes obscured by hazes. On the other hand, layers higher than $600 \mathrm{~km}$ are too tenuous to have a significant effect on the stellar flux. As explained later, however, in this paper we restrict our study to altitudes between $\sim 290$ and $500 \mathrm{~km}(\sim 110-1.4 \mu$ bar $)$, where the signal-to-noise ratio is high enough to reveal fine density fluctuations in Titan's atmosphere.

Unique results have already been obtained from this occultation. A joint analysis of the lightcurves and a detailed discussion of the results were published by Hubbard et al. (1993b), hereafter referred to as H93. Complementary information is presented in the references mentioned at the beginning of the next section. Among the results derived so far, we can quote the temperature profiles between $\sim 300$ and $500 \mathrm{~km}$, the spatial extension of Titan's stratospheric haze (with a north/south asymmetry), and the aerosol size distribution. Noteworthy also is the fortuitous detection of a central flash as the European stations went near the center of Titan's shadow. This flash yields the precise shape of the 250- $\mu$ bar isobar (250-km altitude) and, thus, provides constraints on the satellite zonal wind system, with evidence for a superotation at that level, and velocities of $\sim 170 \mathrm{~m} \mathrm{~s}^{-1}$ at high latitudes, dropping to $\sim 100 \mathrm{~m} \mathrm{~s}^{-1}$ near the equator. For comparison the speed of sound at that level is $\sim 270 \mathrm{~m} \mathrm{~s}^{-1}$, assuming a temperature of $175 \mathrm{~K}$ and a pure nitrogen atmosphere.

Meanwhile, more information is still contained in the data. In particular a conspicuous stellar scintillation was monitored during the whole event and from all the stations. This scintillation is caused by refractivity and thus also density inhomogeneities in Titan's atmosphere. From a theoretical point of view, these features provide us with important information about the dynamical state of the stratosphere. In particular, there has been indication for propagating internal gravity waves from the Voyager radio occultation experiment lower in the atmosphere, i.e., below $\sim 90 \mathrm{~km}$ ( 13 mbar) (see Hinson and Tyler 1983, and Friedson 1994). These waves propagating upward will increase their amplitude, to conserve their energy flux as the background density drops. The regions that we probe are so high that significant fluctuations $( \pm 5 \%)$ can be observed, and serve as tests for general models of Titan's atmospheric dynamics.

Particularly important is the contribution of these waves to the energy and momentum budgets of the upper stratosphere. The momentum deposited when the waves break can serve to maintain zonal winds, while the mechanical energy dissipated through molecular viscosity may compete with the other sources of heat at those altitudes [see the example of Neptune, as described by Roques et al. (1994)]. Also, breaking waves can be an important source of eddy mixing and can cause zonal winds to decrease with height, as is the case in the terrestrial mesosphere and is probably the case above the cloud tops on Venus (Hinson and Jenkins 1995). From a more practical point of view, these inhomogeneities may be important for and have measurable effects on the Huygens probe as it plunges in the satellite atmosphere in December 2004 (Fulchignoni 1992, and see the discussion by Strobel and Sicardy 1997). Conversely, the probe acceleration may serve as a tool to monitor the vertical density profile over a fine scale.

While it is obvious that a significant scintillation is present in our data, some care must be taken when interpreting the results derived from their analysis. The aim of this paper is to evaluate the role of several possible biases that can distort the original structure of the atmosphere. Then, our results can be used as input parameters for further modeling of Titan stratosphere dynamics, a work that we defer to the future.

After a brief description of the observations (Section 2), we define a consistent origin and a consistent altitude scale for all the lightcurves (Section 3). This is necessary when comparing data sets, in particular to study the horizontal structure of the observed features. Density and temperature profiles are derived in Section 4, and fluctuations with respect to a smooth atmosphere are described in Section 5. In that section, we show evidence for breaking structures under convective instability. In Section 6, vertical spectra of the fluctuations are derived. The possible biases due to the finite stellar diameter and ray crossing are 
examined in Section 7. The horizontal layering of the atmosphere is analyzed in Section 8, with a determination of the horizontal-to-vertical ratio (aspect ratio) of the structures and their horizontal spectrum. We finally discuss our results in Section 9.

\section{OBSE RVATIONS}

Observational details and preliminary results can be found in Beisker et al. (1989), Forrest and Nicolson (1990), Hubbard et al. (1990), and Sicardy et al. (1990). More complete description and analysis are provided in H93. We have chosen, among all the available data, the lightcurves with sufficient signal-tonoise ratio and time resolution to reveal the stellar scintillation in Titan atmosphere. The observational circumstances of the data used in this paper are summarized in Table I.

The geocentric ephemeris of Titan, based on the VSOP82 theory (Bretagnon 1982), was provided by the Bureau des Longitudes, and is available on request. The adopted coordinates for $28 \mathrm{Sgr}$ are $\alpha(1950)=.18^{\mathrm{h}} 43^{\mathrm{mn}} 19^{\mathrm{s}} .77, \delta(1950)=.-22^{\circ} 26^{\prime}$ $46^{\prime \prime} .91$. This position takes into account the proper motion of the star (D. Mink, private communication, 1989). Finally, the parallax effects associated with the observing sites on the Earth (Table I) yield the motion of the observer in Titan's shadow. However, the accuracies of the ephemeris and on the stellar position are not sufficient to define the absolute position of an observer in the shadow at a given time. We have to use for that some benchmarks in the satellite atmosphere itself, as described in the next section. These benchmarks provide the position of each observer at a given time and, thus, uniquely define the absolute path of the observers in the satellite shadow.

The resulting tracks are shown in Figs. 1 and 2. We denote $\xi$ and $\eta$ the eastward and northward positions, respectively, of the observer relative to the center of Titan's shadow. The two closest paths corresponding to Northern Europe stations are separated by about $15 \mathrm{~km}$, and the farthest ones (Northern Europe and Israel) are about $1700 \mathrm{~km}$ apart. The dotted circle in Fig. 1 shows the location of the inversion layer "A", at the altitude of

TABLE I

Circumstances of Observations

\begin{tabular}{|c|c|c|c|c|c|}
\hline Site & $\begin{array}{c}\text { Latitude } \\
\text { Longitude } \\
\text { Elevation (m) }\end{array}$ & Personnel & $\begin{array}{l}\text { Telescope } \\
\text { diameter } \\
(\mathrm{cm})\end{array}$ & $\begin{array}{c}\text { Sampling } \\
\text { rate } \\
\text { (s) }\end{array}$ & $\begin{array}{c}\lambda \\
(\mu \mathrm{m})\end{array}$ \\
\hline Manley Obs., UK & $\begin{array}{c}53^{\circ} 14^{\prime} 43^{\prime \prime} \mathrm{N} \\
02^{\circ} 44^{\prime} 15^{\prime \prime} \mathrm{W} \\
75\end{array}$ & $\begin{array}{l}\text { R. Miles } \\
\text { A. J. Hollis }\end{array}$ & 35 & 0.2 & 0.55 \\
\hline Hertford Obs., UK & $\begin{array}{c}51^{\circ} 46^{\prime} 28^{\prime \prime} \mathrm{N} \\
00^{\circ} 0531^{\prime \prime} \mathrm{W} \\
66\end{array}$ & $\begin{array}{l}\text { R. W. Forrest } \\
\text { I. K. M. Nicolson }\end{array}$ & 36 & 0.1 & 0.72 \\
\hline Royal Greenwich Obs., UK & $\begin{array}{c}50^{\circ} 52^{\prime} 10^{\prime \prime} \mathrm{N} \\
00^{\circ} 2051^{\prime \prime} \mathrm{E} \\
50\end{array}$ & G. Appleby & 50 & 0.2 & 0.532 \\
\hline Langwedel, Germany & $\begin{array}{c}52^{\circ} 58^{\prime} 37^{\prime \prime} \mathrm{N} \\
09^{\circ} 11^{\prime} 03^{\prime \prime} \mathrm{E} \\
20\end{array}$ & $\begin{array}{l}\text { M. Bruns } \\
\text { M. Nezel } \\
\text { H. Struckmann }\end{array}$ & 30 & 0.2 & 0.55 \\
\hline Essen, Germany & $\begin{array}{c}51^{\circ} 24^{\prime} 35^{\prime \prime} \mathrm{N} \\
07^{\circ} 04^{\prime} 15^{\prime \prime} \mathrm{E} \\
94\end{array}$ & H. Denzau & 36 & 0.2 & 0.55 \\
\hline Pic du Midi Obs., France & $\begin{array}{c}42^{\circ} 56^{\prime} 12^{\prime \prime} \mathrm{N} \\
00^{\circ} 08^{\prime} 32^{\prime \prime} \mathrm{E} \\
2861\end{array}$ & $\begin{array}{l}\text { J. Lecacheux } \\
\text { S. Pau } \\
\text { B. Sicardy }\end{array}$ & 200 & 0.05 & 0.75 \\
\hline Vatican Obs., Italy & $\begin{array}{c}41^{\circ} 44^{\prime} 48^{\prime \prime} \mathrm{N} \\
12^{\circ} 39^{\prime} 06^{\prime \prime} \mathrm{E} \\
450\end{array}$ & $\begin{array}{l}\text { E. Carreira } \\
\text { F. Rossi }\end{array}$ & 61 & 0.1 & $\begin{array}{l}0.45 \\
0.8\end{array}$ \\
\hline Catania Obs., Italy & $\begin{array}{c}37^{\circ} 41^{\prime} 30^{\prime \prime} \mathrm{N} \\
14^{\circ} 58^{\prime} 42^{\prime \prime} \mathrm{E} \\
1725\end{array}$ & C. Blanco & 91 & 1.0 & 0.44 \\
\hline Ein Harod, Israel & $\begin{array}{c}32^{\circ} 33^{\prime} 35^{\prime \prime} \mathrm{N} \\
35^{\circ} 23^{\prime} 42^{\prime \prime} \mathrm{E} \\
20\end{array}$ & $\begin{array}{l}\text { H. J. Reitsema } \\
\text { Y. Nevo }\end{array}$ & 36 & 0.1 & $\begin{array}{l}0.45 \\
0.80\end{array}$ \\
\hline Wise Obs., Israel & $\begin{array}{c}30^{\circ} 35^{\prime} 48^{\prime \prime} \mathrm{N} \\
34^{\circ} 45^{\prime} 48^{\prime \prime} \mathrm{E} \\
836\end{array}$ & N. Brosch & 100 & 0.1 & 0.73 \\
\hline
\end{tabular}




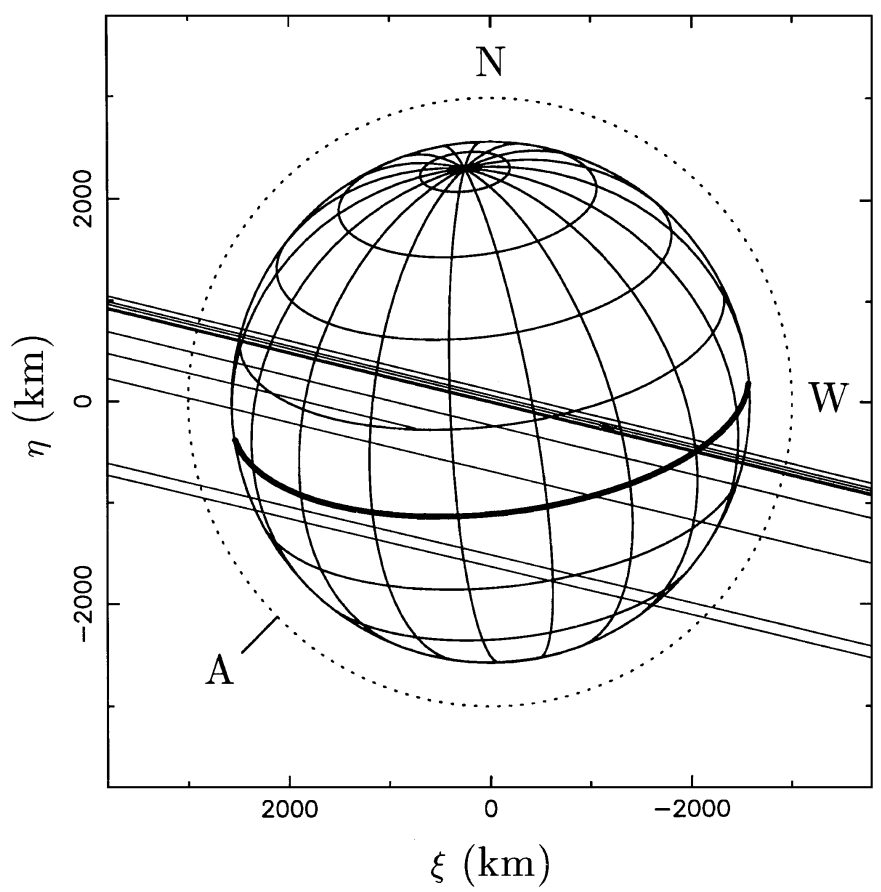

FIG. 1. Tracks of the various stations in Titan's shadow, as projected in the plane of the sky (W and $\mathrm{N}$ denote celestial west and north at epoch). Observers move from the right to the left in this diagram. The lower two tracks correspond to stations in Israel. The upper five tracks, packed together, correspond to stations in Northern Europe; see Fig. 2 for an expanded view. Note that the Vatican track, at the left, starts only halfway in the shadow, due to the presence of clouds during the first half of the occultation. The radius of the solid surface of Titan is taken to be $R_{\mathrm{T}}=2575 \mathrm{~km}$, but it was not detected during these observations (see Fig. 2). The dotted circle marks the location of the inversion layer A, at $425-\mathrm{km}$ altitude, described in the text.

$425 \mathrm{~km}$ (pressure $p \sim 7 \mu$ bar), which is discussed later. The halflight stellar flux was reached when the star was just above that altitude, at about $450 \mathrm{~km}$ above Titan's surface ( $p \sim 4 \mu$ bar). The titanocentric latitudes of the suboccultation points at that level are listed in Table II for each station. This calculation assumes that Titan and Saturn have their poles aligned, as seems to be the case from the analysis of $\mathrm{H} 93$.

The diameter of $28 \mathrm{Sgr}$ (projected at the distance of Titan, $D=1.35 \times 10^{9} \mathrm{~km}$ ) has been derived from the occultation of the star by chosen Saturn's ring sharp edges (French et al. 1993, Hubbard et al. 1993a). This diameter, $\sim 18 \mathrm{~km}$, corresponds to about $1 \mathrm{~s}$ of time in the lightcurves, since the velocity of the observer in Titan's shadow was close to $18.5 \mathrm{~km} \mathrm{~s}^{-1}$ for all the stations. On the other hand, the Fresnel scale $F$ is of the order of $1 \mathrm{~km}$ in the visible (see Appendix A), so that the spatial resolution is dominated by the stellar diameter, not by diffraction effects. Note, however, that as the star "sets" in Titan's atmosphere, its vertical diameter shrinks in proportion to the stellar flux. Consequently, the vertical resolution improves as deeper layers are explored. For instance, at the upper parts of the various profiles shown in this paper (altitude level around $500 \mathrm{~km}$ ) the vertical resolution is about $13 \mathrm{~km}$. At the bottom of the profiles (altitude $\sim 300 \mathrm{~km}$ ), it reduces to about $1.5 \mathrm{~km}$.

\section{AN ABSOLUTE VERTICAL SCALE}

Stellar scintillation is detected not only during ingress and egress (Fig. 3), but also well above the noise during the entire occultation (Fig. 4), as the stellar image follows Titan's limb (Fig. 2).

To study in detail the structure of the atmosphere from various stations, we need a consistent way of associating the time at which a stellar photon is received by an observer at the Earth with the altitude $z$ and position angle of the stellar image in Titan's atmosphere at that time. A failure to do so will misalign atmospheric features that are otherwise connected.

As an observer enters Titan's shadow, the stellar image diverges from the observer's track, due to refraction effects (Fig. 2). We want to know where the image of the star is at each moment along the limb of the satellite. There are actually two problems

TABLE II

Titanocentric Latitudes of the Half-Light Levels and Time Shifts for E ach Station

\begin{tabular}{lcccccc}
\hline \multirow{2}{*}{ Site } & \multicolumn{2}{c}{ Latitude } & & & & \\
\cline { 2 - 4 } & Immersion & Emersion & $\begin{array}{c}\text { Emmersion } \\
\text { (layer A) }\end{array}$ & $\begin{array}{c}\delta t \text { emersion } \\
\text { (layer A) }\end{array}$ & $\begin{array}{c}\delta t \\
\text { (central flash) }\end{array}$ & Average $\delta t$ \\
\hline Manley & $-15.9^{\circ}$ & $+20.0^{\circ}$ & $-0.066 \mathrm{~s}$ & $+0.38 \mathrm{~s}$ & $+0.60 \mathrm{~s}$ & $+0.30 \mathrm{~s}$ \\
Hertford & $-16.8^{\circ}$ & $+19.1^{\circ}$ & $+0.22 \mathrm{~s}$ & $+0.097 \mathrm{~s}$ & $-0.11 \mathrm{~s}$ & $+0.07 \mathrm{~s}$ \\
RGO & $-17.3^{\circ}$ & $+18.7^{\circ}$ & $-0.44 \mathrm{~s}$ & $-0.81 \mathrm{~s}$ & $-0.77 \mathrm{~s}$ & $-0.67 \mathrm{~s}$ \\
Langwedel & $-17.7^{\circ}$ & $+18.1^{\circ}$ & $-0.27 \mathrm{~s}$ & $-0.18 \mathrm{~s}$ & $+0.081 \mathrm{~s}$ & $-0.12 \mathrm{~s}$ \\
Essen & $-18.0^{\circ}$ & $+17.8^{\circ}$ & $+0.25 \mathrm{~s}$ & $+0.088 \mathrm{~s}$ & $-0.13 \mathrm{~s}$ & $+0.07 \mathrm{~s}$ \\
Pic du Midi & $-21.9^{\circ}$ & $+14.0^{\circ}$ & $+0.54 \mathrm{~s}$ & $+0.63 \mathrm{~s}$ & n.a. & $+0.59 \mathrm{~s}$ \\
Vatican & n.a. & $+10.4^{\circ}$ & n.a. & $-0.96 \mathrm{~s}$ & n.a. & $-0.96 \mathrm{~s}$ \\
Catania & $-29.5^{\circ}$ & $+6.0^{\circ}$ & $+0.51 \mathrm{~s}$ & $+0.35 \mathrm{~s}$ & n.a. & $+0.43 \mathrm{~s}$ \\
Ein Harod & $-44.0^{\circ}$ & $-09.4^{\circ}$ & $-0.29 \mathrm{~s}$ & $-0.23 \mathrm{~s}$ & n.a. & $-0.26 \mathrm{~s}$ \\
Wise & $-46.2^{\circ}$ & $-11.7^{\circ}$ & $-0.55 \mathrm{~s}$ & $-0.59 \mathrm{~s}$ & n.a. & $-0.57 \mathrm{~s}$ \\
\hline
\end{tabular}



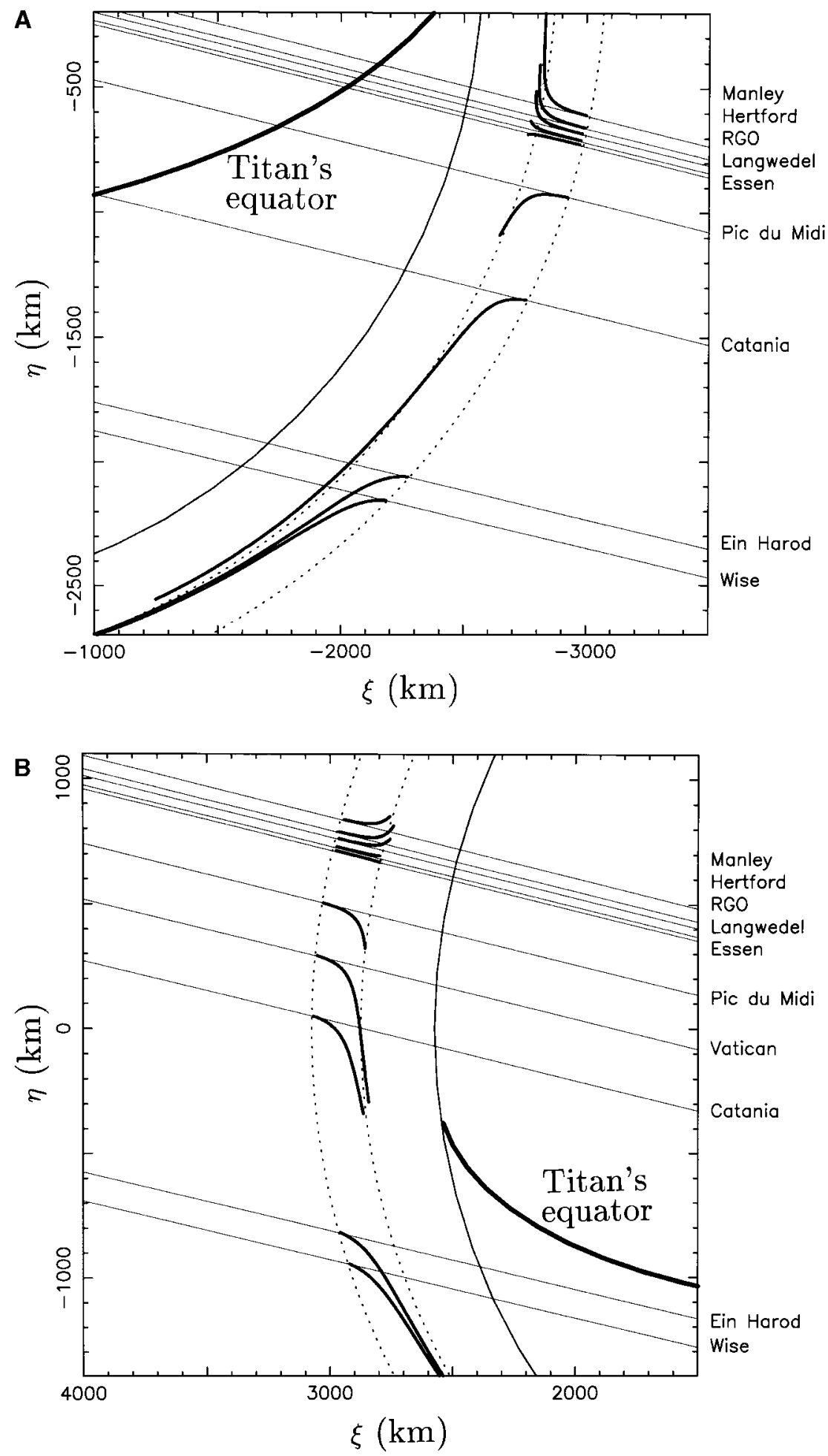

FIG. 2. Closeup views of the tracks shown in Fig. 1, at ingress (A) and egress (B). The thin lines are the tracks followed by the observers in Titan's shadow, as in Fig. 1. The dotted circles show the 300- and 500-km altitude levels, between which the most significant data are recorded. The thick curves are the tracks actually followed by the stellar images along the limb, between these two limits. Note that the stellar images first probe the atmosphere vertically, and then move essentially horizontally. 


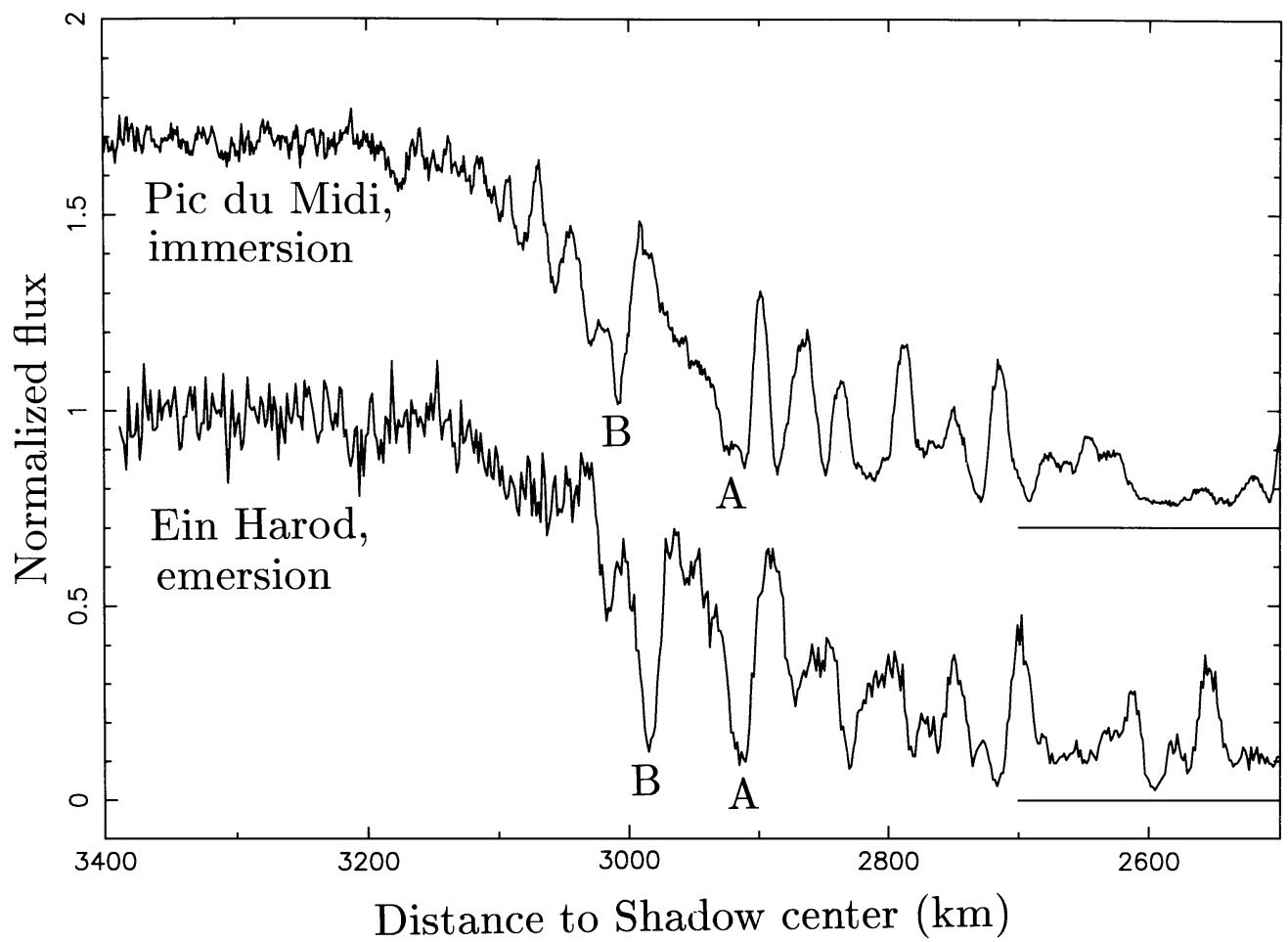

FIG . 3. Fiducial features A and B observed at Pic du Midi and Ein Harod (red channel). These features are used as benchmarks for defining an absolute origin of altitude. The abscissa is the distance from the observer to the center of Titan's shadow. The zero stellar flux for each normalized lightcurve is indicated by the horizontal line on the right. For sake of clarity, the Pic du Midi lightcurve has been shifted vertically by 0.7 .

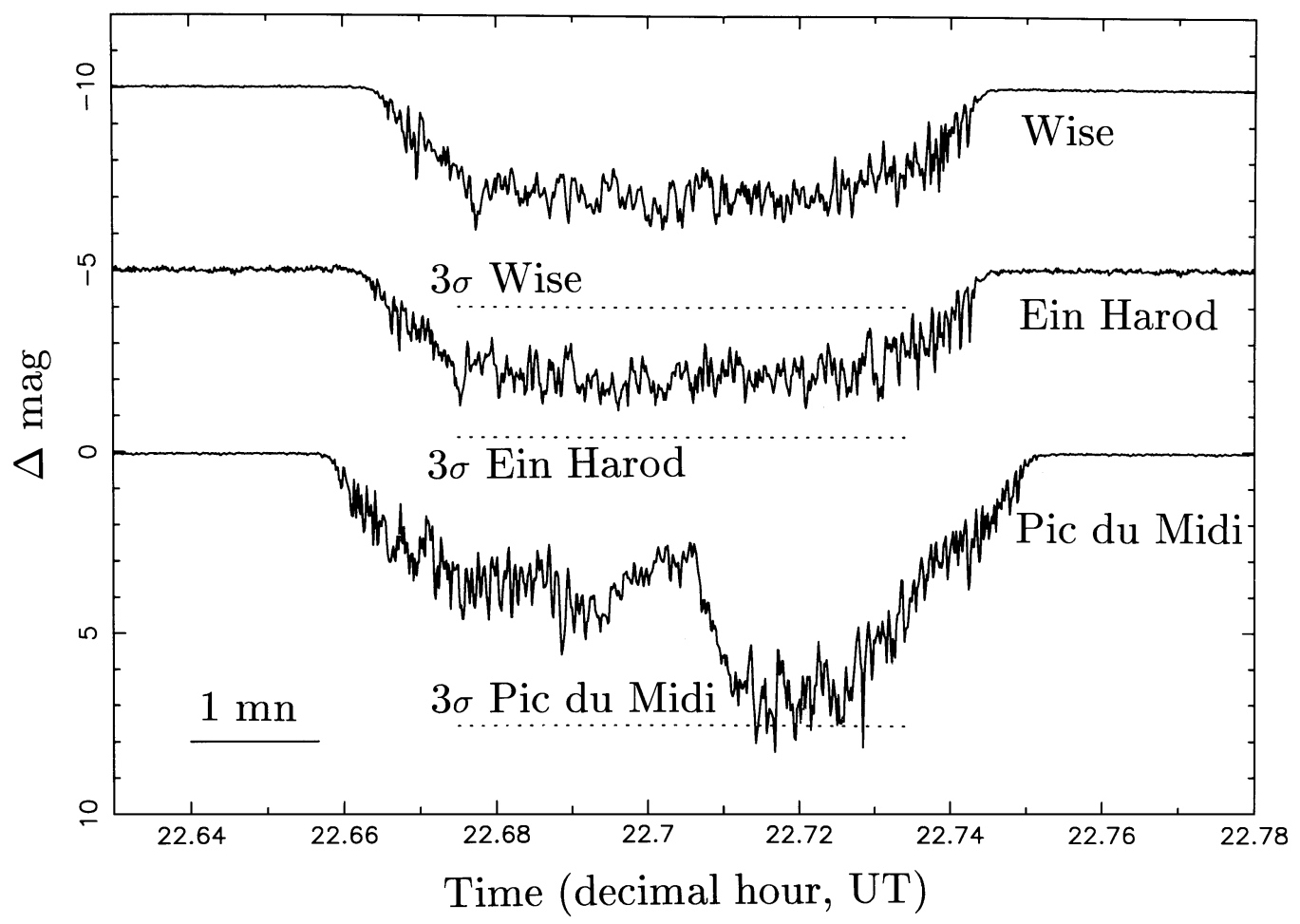

FIG . 4. Drops in magnitude of the stellar flux as a function of time for three chosen stations. The lightcurves have been smoothed at a time resolution of $0.5 \mathrm{~s}$. The time on the abscissa applies to the Pic du Midi lightcurve only; $+148 \mathrm{~s}$ has been added to the Wise and Ein Harod UT times to center all the data in the figure. Note also that for sake of clarity, the Wise and Ein Harod curves have been shifted vertically by -10 and -5 magnitudes, respectively. The dotted lines indicate the drop in magnitude that the star should have at each station to reach the $3 \sigma$ noise level due to the background flux (Titan + sky). Thus, the stellar scintillation due to Titan's atmosphere largely dominates the stellar scintillation due to the Earth atmosphere, except during the second half of the Pic du Midi event. In this part of the lightcurve, the stellar light was almost completely blocked by an aerosol layer (see H93). Note finally the wide central flash observed at Pic du Midi, caused by ray focusing near the shadow center. 
in this calculation: (i) the time recorded at a given station may be wrong, which can translate into several kilometers in Titan's atmosphere at a velocity of $18.5 \mathrm{~km} \mathrm{~s}^{-1}$, and (ii) the position of the stellar image actually depends on the refractivity profile of the atmosphere, which is not known a priori (this is actually what we are looking for!).

\subsection{Origin of Altitudes: The 425-km Inversion Layer}

We have first allowed a small shift in time, $\delta t$, for each station. This shift slides each track along itself by about $18.5 \mathrm{~km} \mathrm{~s}^{-1}$, as noted before. Unfortunately, we cannot use the solid surface of the satellite as a benchmark to determine $\delta t$, since refraction prevents the image of the star from reaching Titan's ground (Fig. 2). However, we can rely on fiducial features in the atmosphere itself.

A first possibility is to use the half-light times derived from isothermal fits to the lightcurves, and then use the corresponding positions in the plane of the sky to derive the shape and the center of the "half-light" limb. Unfortunately, the half-light times can hardly be derived to better than about $\pm 0.5 \mathrm{~s}$, i.e., about $10 \mathrm{~km}$ in the plane of the sky. This is due to the fact that some data are intrinsically noisier, or have poorly calibrated background (Titan + sky) levels. Also, the aerosol layer severely distorts some of the lightcurves, providing displaced half-light times (see, for instance, the emersion at Pic du Midi in Fig. 4). Modeling the effects of the aerosols on the lightcurves is a rather difficult task since this layer is not homogeneous along the limb, and also because its absorbing properties depend on wavelength (see H93).

Consequently, we prefer to use more local and sharp features in the lightcurves. They have the advantage of providing timings that are much less affected by possible absorptions and/or calibration problems.

We noted in particular that a conspicuous broad spike delimited by two sharp dips (denoted "A" and "B") is present in all the lightcurves (Beisker et al. 1989) (and see two examples in Fig. 3). We see later that these dips correspond to inversion layers in the temperature profiles (see, for instance, Figs. 7 and 9). We first projected, in Titan's shadow, the positions of the observers at the times when these dips were observed. A leastsquares adjustment then provides the center of the circle that best fits all these positions, allowing for the small shifts $\delta t$ in the timing of each station (see Table II). We use only the positions provided by dip A, because dip B is not so well defined in some stations (see Figs.18-20). Because it is higher in altitude, the signature of dip B is relatively more affected by noise, which explains probably why its altitude seems to vary by a few kilometers even for nearby stations (see, for instance, the Wise and Ein Harod profiles in the Fig. 20B).

Another sharp benchmark is provided by the central flash, when available. At the northernmost stations (Manley, Hertford, RGO, Langwedel, and Essen), the central flash is a narrow spike, which is thus quite sensitive to timing errors (see H93). The global solution obtained for the central flash in $\mathrm{H} 93$ does show small discrepancies between the model and the actual observations. The $\delta t$ values necessary to bring the central flash observations onto the $\mathrm{H} 93$ model are listed in Table II. One can note the clear correlation of the $\delta t$ values obtained from layer $\mathrm{A}$ at immersion and emersion and from the central flash. Unless coincidental, this correlation strongly points toward timing errors, rather than a physical departure of layer A from a circle.

The times for feature A are listed in Table III, as are the average $\delta t$ values that have been applied to each station (see also the last column of Table II). Once these shifts have been taken into account, we obtain in the last two columns of Table III the positions $\xi$ and $\eta$ of the observers in the shadow plane, using the ephemeris of Titan, the coordinates of the star, and the parallax effect for each station, as described at the beginning of Section 2.

A circular least-squares fit to these positions yields a radius of $2910.3 \mathrm{~km}$ in the shadow plane for feature A, with a rms deviation of $2.5 \mathrm{~km}$ (excluding the Vatican station, see the comments below), and a center at $\xi_{\mathrm{c}}=-8143.6 \mathrm{~km}$ and $\eta_{\mathrm{c}}=-660.5 \mathrm{~km}$. This center is then taken as the origin of coordinates in the shadow plane, in particular for calculating the distance of the suboccultation points to Titan's center.

\section{TABLE III G eometry of Layer A}

\begin{tabular}{|c|c|c|c|c|c|}
\hline \multirow[b]{2}{*}{ Site } & \multirow{2}{*}{$\begin{array}{l}\text { Immersion } \\
\text { (UT) }\end{array}$} & \multirow{2}{*}{$\begin{array}{l}\text { Emersion } \\
\text { (UT) }\end{array}$} & \multirow{2}{*}{$\begin{array}{l}\text { Time shift } \\
\text { (s) }\end{array}$} & \multicolumn{2}{|c|}{$\begin{array}{c}\text { Shadow-plane } \\
\text { coordinates } \xi, \eta(\mathrm{km})\end{array}$} \\
\hline & & & & Immersion & Emersion \\
\hline Manley & $22: 39: 30.5$ & $22: 44: 43.5$ & +0.30 & $\begin{array}{r}-10,992.2 \\
-1,222.0\end{array}$ & $\begin{array}{r}-5350.3 \\
+150.1\end{array}$ \\
\hline Hertford & $22: 39: 24.1$ & $22: 44: 37.6$ & +0.07 & $\begin{array}{r}-10,991.6 \\
-1,273.0\end{array}$ & $\begin{array}{r}-5335.2 \\
+100.5\end{array}$ \\
\hline RGO & $22: 39: 24.9$ & $22: 44: 38.7$ & -0.67 & $\begin{array}{r}-10,987.3 \\
-1,298.5\end{array}$ & $\begin{array}{r}-5325.5 \\
+75.8\end{array}$ \\
\hline Langwedel & $22: 38: 49.9$ & 22:44:03.4 & -0.12 & $\begin{array}{r}-10,973.5 \\
-1,326.0\end{array}$ & $\begin{array}{r}-5318.2 \\
+41.5\end{array}$ \\
\hline Essen & $22: 38: 59.0$ & $22: 44: 12.6$ & +0.07 & $\begin{array}{r}-10,976.1 \\
-1,343.4\end{array}$ & $\begin{array}{r}-5316.0 \\
+26.1\end{array}$ \\
\hline Pic du Midi & $22: 39: 40.5$ & $22: 44: 52.2$ & +0.59 & $\begin{array}{r}-10,913.6 \\
-1,548.6\end{array}$ & $\begin{array}{r}-5274.3 \\
-181.3\end{array}$ \\
\hline Vatican (red) & n.a. & $22: 44: 00.7$ & -0.96 & $\begin{array}{l}\text { n.a. } \\
\text { n.a. }\end{array}$ & $\begin{array}{r}-5245.8 \\
-395.1\end{array}$ \\
\hline Catania & $22: 38: 46.3$ & $22: 43: 50.5$ & +0.43 & $\begin{array}{r}-10,748.0 \\
-1,962.4\end{array}$ & $\begin{array}{r}-5232.4 \\
-638.0\end{array}$ \\
\hline Ein Harod (red) & $22: 37: 31.5$ & $22: 42: 00.9$ & -0.26 & $\begin{array}{r}-10,247.3 \\
-2,670.4\end{array}$ & $\begin{array}{l}-5361.0 \\
-1511.4\end{array}$ \\
\hline Wise & $22: 37: 38.5$ & $22: 42: 00.4$ & -0.57 & $\begin{array}{r}-10,154.4 \\
-2,764.2\end{array}$ & $\begin{array}{l}-5402.1 \\
-1637.4\end{array}$ \\
\hline
\end{tabular}

${ }^{a}$ Once the time shifts have been applied; see text. 
The validity of our procedure can be addressed, in particular the assumption that the fiducial feature A (and, to a lesser extent, B) corresponds to a global spherical horizontal layer in the satellite atmosphere. This assumption can actually be validated on several grounds:

i. The rms residual per degree of freedom is significantly improved once we apply the time-corrected $\delta t$ values. We have actually 18 points along the limb, while a circular fit has three free parameters (position of the center and radius). The rms residual of the circular fit without time shift is $7.5 \mathrm{~km}$, corresponding to a rms residual per degree of freedom of $7.5 \sqrt{18 /(18-3)}=$ $8.2 \mathrm{~km}$. If time shifts are allowed for each of the nine stations, we have then 12 free parameters (nine time shifts and three parameters for the circle). Since the rms residual for the circular fit is $2.5 \mathrm{~km}$, this yields a rms residual per degree of freedom of $2.5 \sqrt{18 /(18-12)}=4.3 \mathrm{~km}$, i.e., an improvement of almost a factor of 2 compared with the fit without time shift. Also, and as noted before, the good correlation observed for the $\delta t$ values derived separately at immersion and emersion and for the central flash strongly suggests systematic timing errors at each station.

ii. The center of the $2910.3-\mathrm{km}$ circle lies only $2.6 \mathrm{~km}$ from the center of the shadow as derived independently from the central flash analysis by H93. The isobar level associated with the central flash is at $p \sim 250 \mu$ bar, while the isobar corresponding to $\operatorname{dip} \mathrm{A}$ is at $p \sim 7 \mu$ bar. The two isobars are thus concentric to within a small fraction of a stellar diameter $(18 \mathrm{~km})$, quite a good agreement if one remembers that these two pressure levels are separated by more than 3.5 scale heights.

iii. As detailed below, no global deviation from a spherical shape is observed, at least at the latitudes probed here $\left(\sim-46^{\circ}\right.$ to $+20^{\circ}$ ), indicating that the $7-\mu$ bar isobar is not significantly oblate in this range of latitude. This is consistent with the shape of the $250-\mu$ bar isobar, as derived from the central flash analysis, which shows a deviation of less than $3 \mathrm{~km}$ from a sphere in the same range of latitude (see Fig. 34 of H93). Thus, although H93 gives a rather substantial global oblateness, $\sim 0.016$, for the 250 - $\mu$ bar level, its departure from a sphere is significant only for latitudes larger (in absolute value) than $\sim 50^{\circ}$.

More quantitatively, we have performed elliptical leastsquares fits to the positions listed in Table III, to detect a possible oblateness of layer A. These fits do not improve the residuals per degree of freedom. For instance, with no time shift applied, we get a rms residual of $7.4 \mathrm{~km}$ with an elliptical fit, not an improvement with respect to $7.5 \mathrm{~km}$ for a circular fit since we now have five free parameters for the ellipse (position of the center, $\xi_{\mathrm{c}}, \eta_{\mathrm{c}}$, semimajor axis, oblateness, position angle), instead of three for the circle. We get a similar result when time shifts are applied: the rms residual is about the same $(2.5 \mathrm{~km})$ using an elliptical or a circular fit. This means that no significant departure from a circle can be detected for layer A, using our data set.
It remains to explain the origin of the timing shifts listed in Table II. According to the observers at Manley, Hatfield, Langwedel, Essen, Pic du Midi, and Catania, these shifts are comparable to or only slightly larger than the announced timing accuracies. It is hard to conclude in the case of the Vatican data, since only one side of the occultation is available. The time shift there is close to $1 \mathrm{~s}$, but it is impossible to check whether a similar correction applied at immersion, to confirm a timing problem. Finally, after discussions with the observers at RGO, Ein Harod, and Wise, no clear explanations can be given for the large (with respect to the announced accuracies) timing corrections that must be applied at these stations.

Finally, we point out that the rms residual of $2.5 \mathrm{~km}$ obtained with a circular fit, and with the time shifts $\delta t$ values applied, corresponds to an internal rms time residual of about $0.13 \mathrm{~s}$, with a velocity of about $18.5 \mathrm{~km} \mathrm{~s}^{-1}$. This is what we can reasonably expect when measuring the times for feature $\mathrm{A}$ and for the central flashes.

In view of the general discussion given above, we thus assume that the fiducial features A and B are consistent with the existence of global spherical layers in Titan's atmosphere, at least at the latitudes probed by the occultation (see Fig. 1, Table II). We shall see that these features actually correspond to two inversion layers, i.e., sudden and local increases in temperature with altitude. Owing to the bending of the stellar rays by refraction, ${ }^{2}$ which amounts to $90 \mathrm{~km}$ at the Earth for layer A, the radius of the circle quoted above, $2910.3 \mathrm{~km}$, places that layer at an altitude of $z_{\mathrm{A}}=2910.3+90-R_{\mathrm{T}}=425.3 \mathrm{~km}$ above the ground, where we use a radius of $R_{\mathrm{T}}=2575 \mathrm{~km}$ for Titan's solid surface (Lindal et al. 1983). A similar procedure yields an altitude of $450-455 \mathrm{~km}$ for layer B. We shall see, however, that this layer tends to disappear at titanocentric latitudes smaller than $-20^{\circ}$ (Fig. 20). From now on, and for each profile, the origin of altitude is thus chosen so that the layer A is exactly at $425 \mathrm{~km}$ above Titan's surface. As discussed in Section 4, our procedure should not introduce systematic errors much larger than $\sim 10-15 \mathrm{~km}$ for this chosen origin of altitude.

\subsection{Defining a Consistent Altitude Scale}

Defining an origin of altitude as explained above is not sufficient, however, to obtain a consistent altitude scale for all the observations. As an observer gets deeper into Titan's shadow, the ray bending due to refraction becomes larger. The resulting defocusing of light is the main cause for the drop of signal in the lightcurves. As long as haze absorption can be ignored (which is not always the case here, see below and Fig. 4), conservation of energy relates the normalized stellar flux $\Phi(t)$ at time $t$ to the altitude interval $d r$ probed by the image of the star in the atmosphere and to the interval of radius $d r^{\prime}$ traveled by the observer

\footnotetext{
${ }^{2}$ The relativistic bending caused by Titan's mass amounts to only $0.2 \mathrm{~km}$ at the Earth, and is negligible here.
} 
in the shadow during the same time:

$$
\Phi(t)=f \frac{d r}{d r^{\prime}}
$$

where $f$ is the focusing factor due to the limb curvature (see Appendix A for notation and diagram).

The variations $d r^{\prime}$ are derived from the motion of the observer in Titan's shadow. The numerical integration of Eq. (1) then yields in principle the altitude $z=r-R_{\mathrm{T}}$ at any time $t$. In reality, any small error in the normalization of $\Phi(t)$, due for instance to imperfect knowledge of the zero stellar flux baseline, will propagate in the integration and systematically distort the altitude scale. Furthermore, noise in $\Phi(t)$ due to the detectors and/or to the Earth's atmosphere will place a given event at the wrong altitude. Finally, absorption by aerosols will reduce $\Phi(t)$ below its expected value in the deeper parts of the lightcurves, distorting again the altitude scale. Such absorption is indeed present in several lightcurves, especially at egress (see Fig. 4 and $\mathrm{H} 93$ ).

To avoid such distortions, we have assumed a given smooth density profile $n(z)$ for the atmosphere, using the model of Yelle (1991) (see Fig. 5). This density profile yields in turn a refractivity profile $v(z)$ (where $1+v$ is the refractive index), assuming that the atmosphere is pure molecular nitrogen, ${ }^{3} \mathrm{~N}_{2}$. We adopt the value $K=1.091 \times 10^{-23}+6.282 \times 10^{-26} / \lambda^{2} \mathrm{~cm}^{3}$ molecule ${ }^{-1}$ for the specific refractivity of nitrogen, where $\lambda$ is expressed in micrometers (Washburn 1930). Assuming a spherically symmetric atmosphere, the profile $v(z)$ provides the bending angle $\omega(z)$ of the stellar ray which went through the layer at altitude $z$ at closest approach to Titan's ground (i.e., at $r=R_{\mathrm{T}}+$ $z$ from the center of the satellite),

$$
\omega(z)=\int_{-\infty}^{+\infty} \frac{\partial v}{\partial r} \cdot d s,
$$

where the integration is carried out on elementary paths $d s$ along the ray [see Appendix A, Eq. (A1)]. Note that $\omega$ is negative. For practical purposes, the integral above has been performed numerically by considering elementary atmospheric layers of thickness $1 \mathrm{~km}$, distributed between the altitudes 250 and $1300 \mathrm{~km}$, with a density profile following Yelle's model. Because the profile $v(z)$ varies almost exponentially with altitude, most of the bending angle $\omega$ comes from a layer of thickness $H$ (the scale height, $\sim 50 \mathrm{~km}$, see below), which corresponds to a horizontal length traveled by the ray of $L \sim \sqrt{2 \pi r H} \sim 1000 \mathrm{~km}$, using $r \sim 3000 \mathrm{~km}$.

Once the function $\omega(z)$ has been tabulated numerically, one can relate (i) the altitude $z$ of the lowest layer probed by a given ray to (ii) the actual distance $r^{\prime}$ of the observer to the center of

\footnotetext{
${ }^{3}$ Argon may also be present in the atmosphere. Its refractivity is so close to that of nitrogen that the results would not be modified.
}

the shadow when this ray is intercepted on the Earth:

$$
z=r^{\prime}-D \cdot \omega(z)-R_{\mathrm{T}}
$$

An interpolation gives $z$ for each position $r^{\prime}$ of the observer in the shadow, yielding for instance the stellar tracks shown in Fig. 2. This procedure has the advantage of providing a smooth and consistent altitude scale, independent of the noise or normalization problems in the lightcurves. We have tested this model by taking another atmospheric model, developed earlier by Lellouch et al. (1990) (see Fig. 5). The resulting paths of the stellar images are almost undistinguishable from those derived from Yelle's profile. The discrepancy reaches only $5 \mathrm{~km}$ in absolute altitude at the bottom of the profiles.

\section{DENSITY AND TEMPERATURE PROFILES}

The primary result of the inversions of the lightcurves is the refractivity profile $v(z)$, from which a number density profile $n(z)$ is derived once a composition is assumed (pure nitrogen in our case, as noted before).

Figures 5 and 6 show the density profiles obtained from various stations, where the altitude is determined by the inversion itself, not from Yelle's model (as will be the case for the other profiles presented in this paper). The decrease in the slope observed at the bottom of some profiles (see Fig. 6) comes from the presence of the aerosol layer. There is, on the other hand, a general divergence of the profiles above $510 \mathrm{~km}$, due to the the exponential decay of the atmospheric density and, thus, to the exponential sensitivity of the profiles to the noise in the lightcurves.

Although there is good general agreement between the theoretical and retrieved profiles (Fig. 5), it is hard to decide, from Fig. 6, which model fits better the observations. Most of the dispersion observed in Fig. 6 is due to the inversion itself (noise, bad knowledge of the zero stellar flux, etc.). This is suggested by the fact that nearby stations, or even multichannel experiments at the same site, can give altitude discrepancies as high as 10-15 km for a given value of the density. In particular, to within $10-15 \mathrm{~km}$ (i.e., 20-30\% of the scale height), we are unable to detect in 1989 a possible change in the surface pressure that would shift the altitude scale by a constant amount with respect to the theoretical model based on the Voyager results of 1980. Such a change in pressure could be due to a variable amount of dissolved nitrogen in hypothetical oceans of ethane or methane on the satellite surface (Dubouloz et al. 1989), although no definitive work has been published concerning this issue (E. Lellouch, 1999, private communication). In any case, our results show that the absolute altitude scale should not have systematic errors much larger than $10-15 \mathrm{~km}$.

Figure 6 shows that the observed density profiles $n(z)$ are basically exponential. Linear fits to the profiles $\log [n(z)]$ between 330 and $470 \mathrm{~km}$ (where the data are less affected by aerosols or noise) yield density scale heights of $H=50 \pm 2 \mathrm{~km}$ 


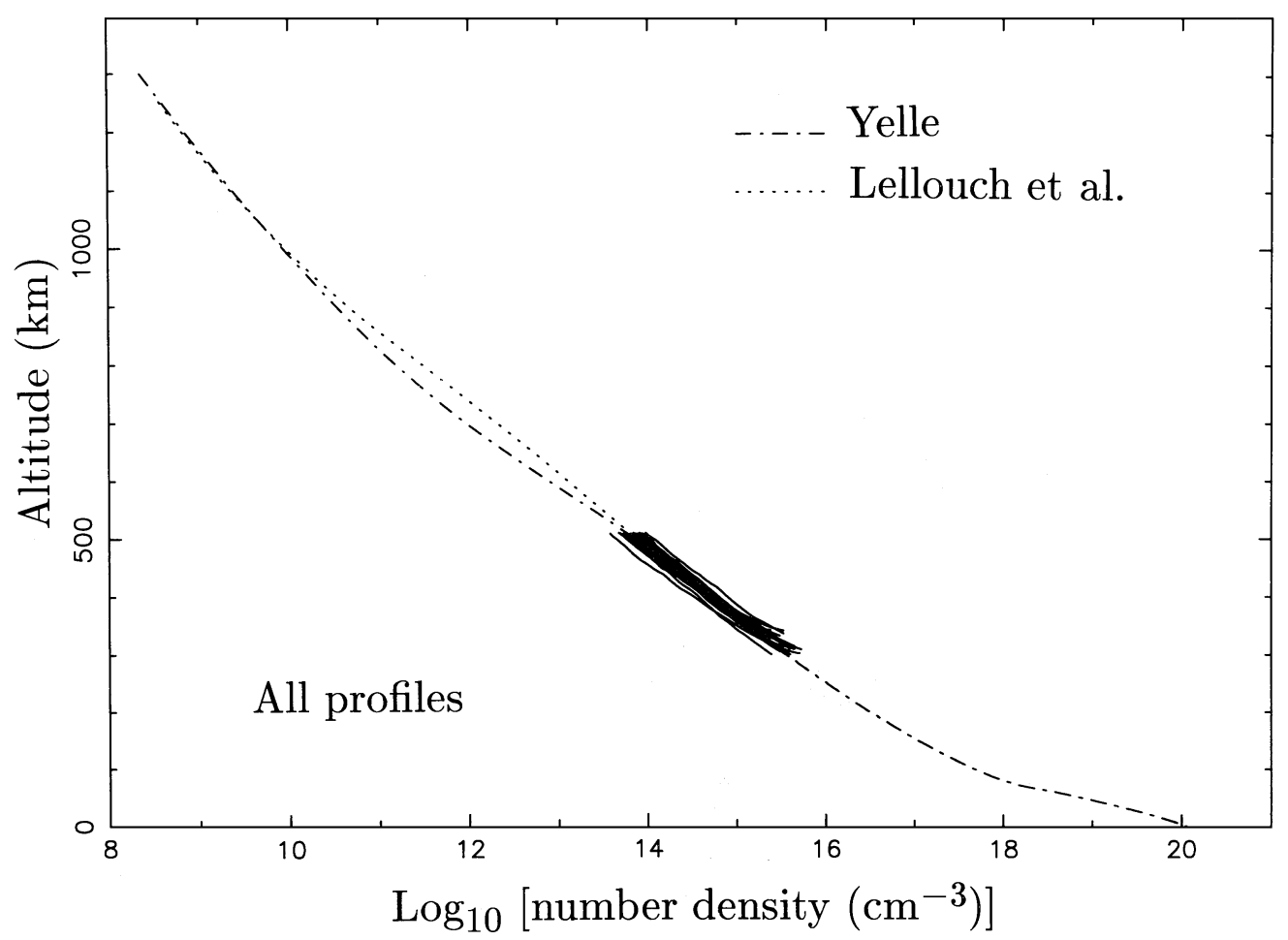

FIG. 5. Theoretical density profiles $n(z)$ derived from the model of Yelle (1991) and Lellouch et al. (1990), compared with the observations (22 profiles between $\sim 300$ and $510 \mathrm{~km}$ ). See details in Fig. 6 . The altitude in this figure and Fig. 6 is determined by the inversion.

and $H=51 \pm 2 \mathrm{~km}$ at immersion and emersion, respectively. We do not note any significant difference in $H$ between immersion and emersion.

To avoid the problems caused by the aerosol layer, in particular concerning the determination of the altitude at the bottom of the profiles, the altitude scale will be, from now on, determined by assuming that the background atmosphere follows a density profile as calculated by Yelle (1991) (see Section 3.2). The other quantities, such as the number density $n$, the temperature $T$, and the temperature gradient $\partial T / \partial z$, will be given by the classic inversion method, as described in Vapillon et al. (1973) and Roques et al. (1994). Thus, only at the moment of plotting the profiles do we use the altitude scale derived from Yelle's model.

The temperature profiles $T(z)$ are derived in particular using an arbitrary initial condition $T=T_{0}$ at $z=z_{0}$. The resulting profiles are shown in Fig. 7, where the initial condition has been chosen so as to coincide with the model of Lellouch et al. at a given altitude. Thus, the apparent better agreement of the observations with this model in Fig. 7 is artificial. In reality, the two models are indistinguishable, as was already evident in Fig. 5 or 6 (see also the discussion of this point in H93, Section 3 ). Note the presence of inversion layers A and B at 425 and $450 \mathrm{~km}$, respectively. These layers will be more conspicuous in the density fluctuation profiles, as we will see now.

\section{DENSITY AND TEMPERATURE FLUCTUATIONS}

Once the exponential trend $n_{\exp }$ has been subtracted, one obtains the relative density fluctuations $\epsilon=\delta n / n_{\exp }=\left(n-n_{\exp }\right) /$ $n_{\text {exp }}$ as a function of altitude (see Fig. 8). Note that the inversion layers at 425 and $450 \mathrm{~km}$ (zone of maximum positive temperature gradients) correspond to negative density fluctuation gradients. We have plotted in Fig. 8 the growth with altitude expected if these fluctuations were caused by freely upward-propagating gravity waves. In the absence of dissipation or zonal wind, this growth ensures that the product of the exponential background density by the amplitude squared of the wave (in other words, its energy flux) is conserved, which translates into the growth factor of $\exp (z / 2 H)$ shown in Fig. 8.

It is difficult to derive any conclusion about such an amplification by examining Fig. 8, first because the lower levels are severely affected by haze absorptions, and second because the upper levels are exponentially affected by noise. In any case, the amplitudes reached by the fluctuations bring them to the limit of convective stability, as we see next. Thus, further growth is probably inhibited above $\sim 500 \mathrm{~km}$. Note that the largest fluctuations (corresponding to layers A and B) have amplitude of $\sim \pm 5 \%$.

Instead of plotting the temperature fluctuations $\delta T=T-\bar{T}$ with respect to an average value $\bar{T}$, we study a physically more 

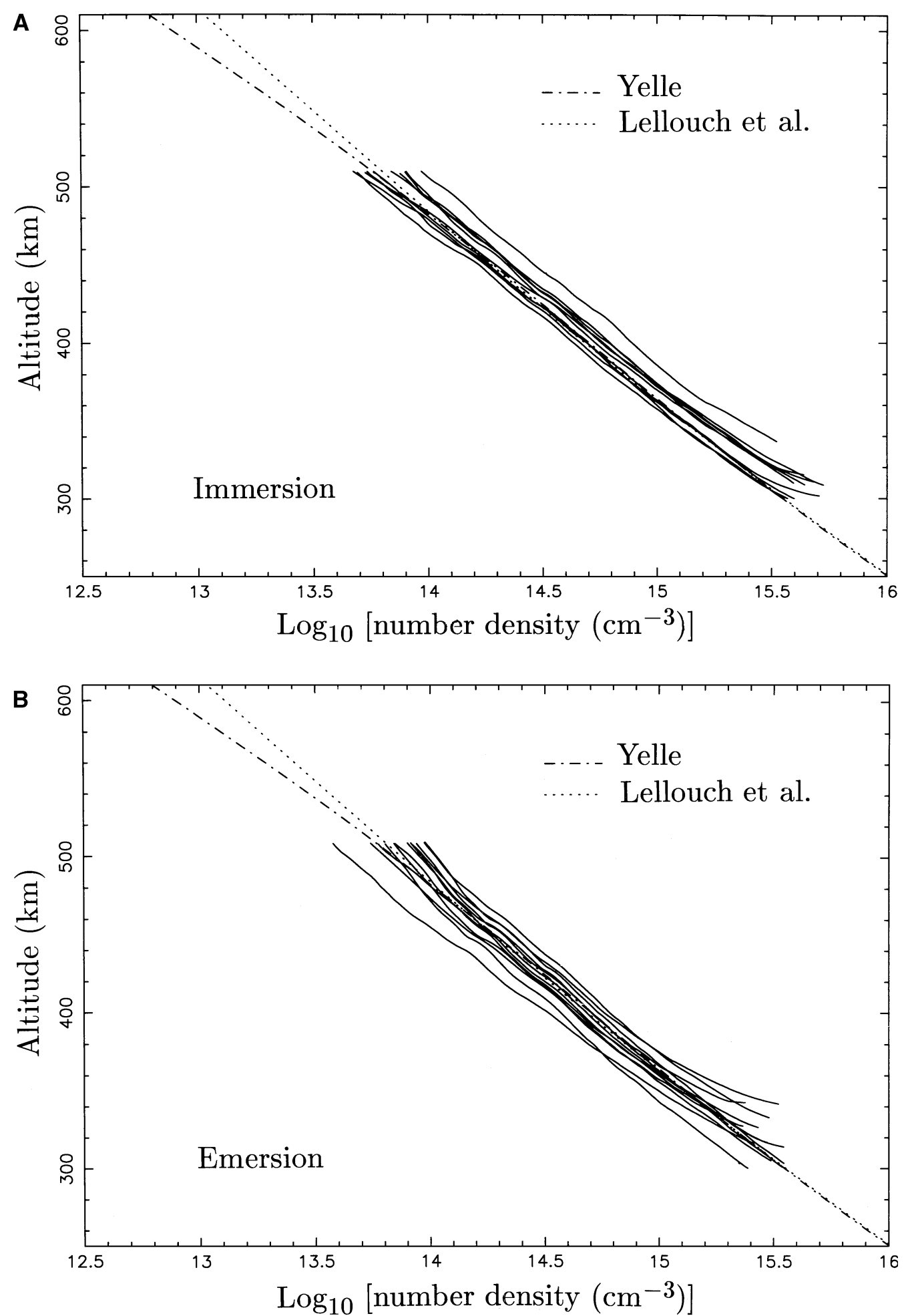

FIG. 6. Enlargments of the previous figure for immersion only (A, 10 profiles) and emersion only (B, 12 profiles). 

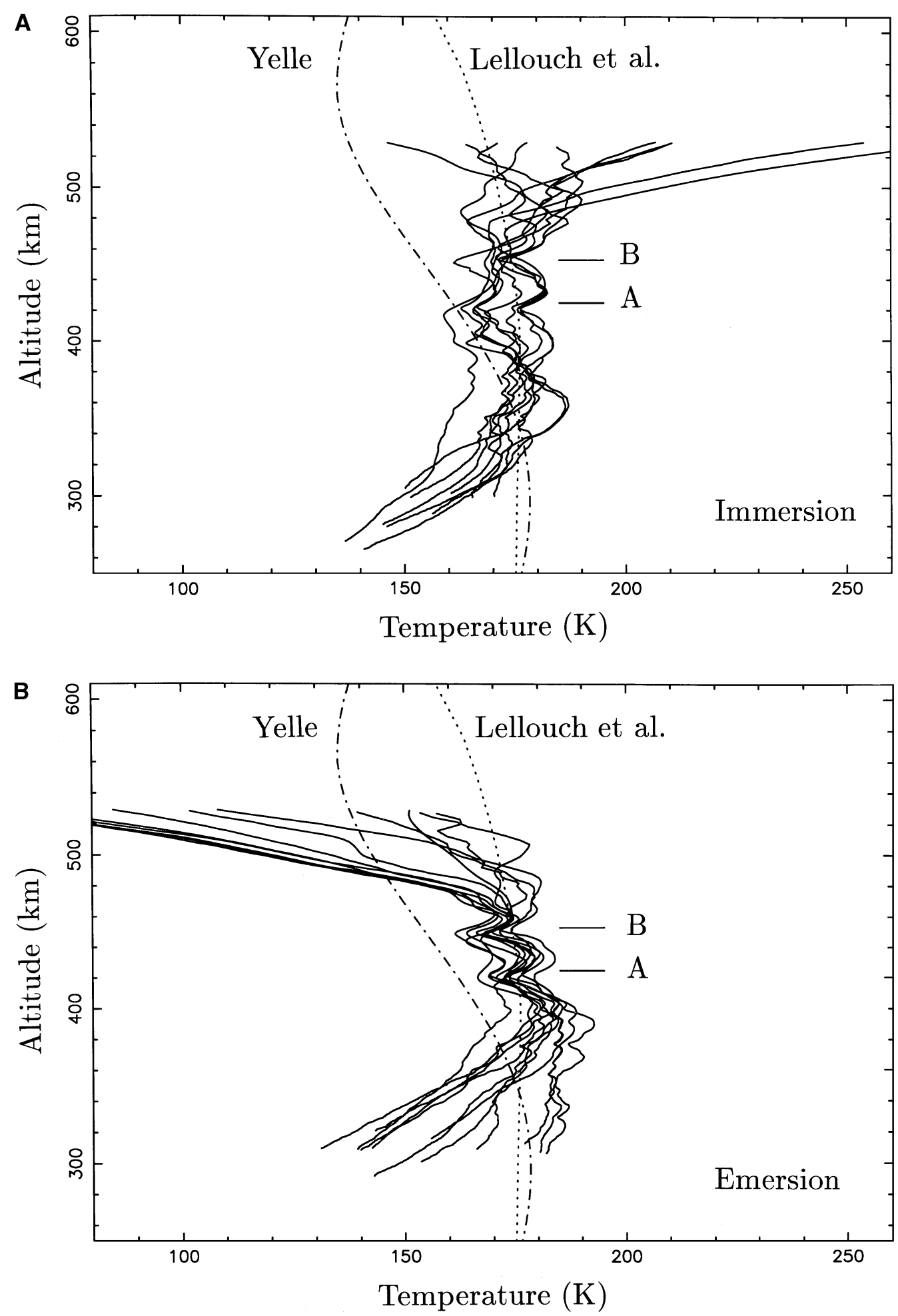

FIG. 7. Temperature profiles derived from the observations at immersion (A) and emersion (B), compared with the models of Yelle and Lellouch et al. The altitude here and in all the remaining profiles is determined by using Yelle's model; see text. The better agreement with the model of Lellouch et al. comes from the arbitrary choice of initial conditions; see the text. Note the systematic drop in temperature at the bottom of the profiles, especially in some emersion profiles. This is caused by the absorbing aerosol layer, and does not correspond to a real decrease in temperature; see H93 for details. 

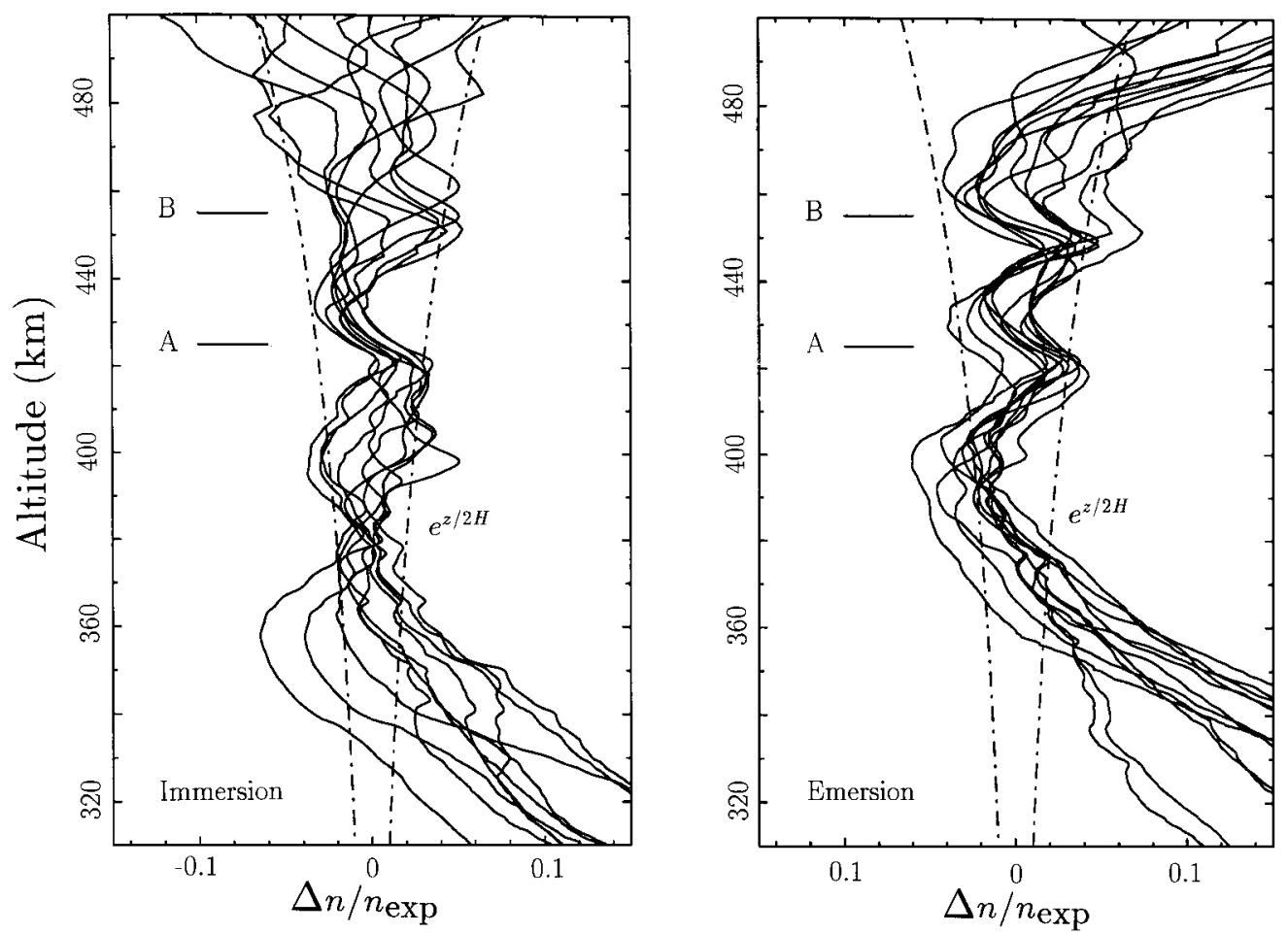

FIG . 8. Relative density fluctuations $\delta n / n_{\exp }$ with respect to an exponential atmosphere at immersion (left) and emersion (right). Only the altitude range $310-500 \mathrm{~km}$ is plotted here, because the higher levels are too much affected by noise to be reliable, while the lower levels are too much obscured by aerosols, which explains the trends of the profiles below $\sim 370 \mathrm{~km}$ (see also Fig. 7). The dash-dotted line indicates the growth of fluctuations expected from freely upward propagating gravity waves.

relevant quantity, namely, the vertical temperature gradient $\partial T /$ $\partial z$. The latter has the advantage of being readily comparable to the adiabatic lapse rate,

$$
\Gamma=-\frac{g}{c_{\mathrm{p}}}
$$

where $g$ is the acceleration of the gravity and $c_{\mathrm{p}}$ is the specific heat at constant pressure. From $g=135.4 \mathrm{~cm} \mathrm{~s}^{-2}$ at the ground, one gets $g=101.4 \mathrm{~cm} \mathrm{~s}^{-2}$ at $400 \mathrm{~km}$. Furthermore, taking $c_{\mathrm{p}}=1.04 \times 10^{7} \mathrm{erg} \mathrm{K}^{-1} \mathrm{~g}^{-1}$, we get $\Gamma \sim-1 \mathrm{~K} \mathrm{~km}^{-1}$ in the region of interest. For larger negative gradients (in absolute value), the atmosphere becomes convectively unstable.

The temperature gradients are shown in Fig. 9. One can see the cutoff of the gradient near the adiabatic lapse rate $\Gamma$, as well as the asymmetry between the negative and positive parts of the profile. The negative parts have a rounder shape sharply bounded by $\Gamma$, while the positive part has a more "spiky" aspect and extends at several times the value of $|\Gamma|$. This behavior is typical of all the temperature gradient profiles. Actually none of them shows a superadiabatic lapse rate.

The cutoff and the asymmetry of the profiles are illustrated in Fig. 10, where the histogram of $\partial T / \partial z$ for selected profiles is plotted. We have eliminated from the histogram the values derived from the Catania station, because of the large time step of $1 \mathrm{~s}$ used there (Table I), which severely smoothes out the temperature profiles. We also eliminated profiles derived in the blue on multichannel detectors, because the aerosol absorption is much more severe in that band than in the red.

\section{SPECTRA OF DENSITY AND TEMPERATURE FLUCTUATIONS}

To better address the question of the nature of the fluctuations observed in Fig. 8, we have performed a Fourier analysis of some of the profiles $\epsilon(z)=\delta n / n_{\exp }(z)$. More precisely, we calculate the power spectra ${ }^{4} P(m)=|\tilde{\epsilon}(m)|^{2}$ of the profiles, where $\tilde{\epsilon}(m)$ is the Fourier transform of $\epsilon(z)$, and where $m=2 \pi / \lambda_{z}$ is the vertical wavenumber. The derivation of $P(m)$ is described in Appendix B, where we note in particular that no spectral information for vertical wavelengths $\lambda_{z}$ larger than $\sim 50 \mathrm{~km}$ and smaller than $\sim 3 \mathrm{~km}$ can be gathered from our profiles.

To avoid as much as possible the effect of aerosol absorptions, we have discarded the profiles derived from the blue channel of some instruments. We have assumed that all the features visible in Fig. 8 are caused by a perfectly layered atmosphere, i.e., that the fluctuations are caused by vertical structures only. This may not be the case when the stellar track in the atmosphere becomes closer to the horizontal (see Fig. 2). To avoid this problem, the

\footnotetext{
${ }^{4}$ Often referred to as "spectra" in the rest of the text.
} 


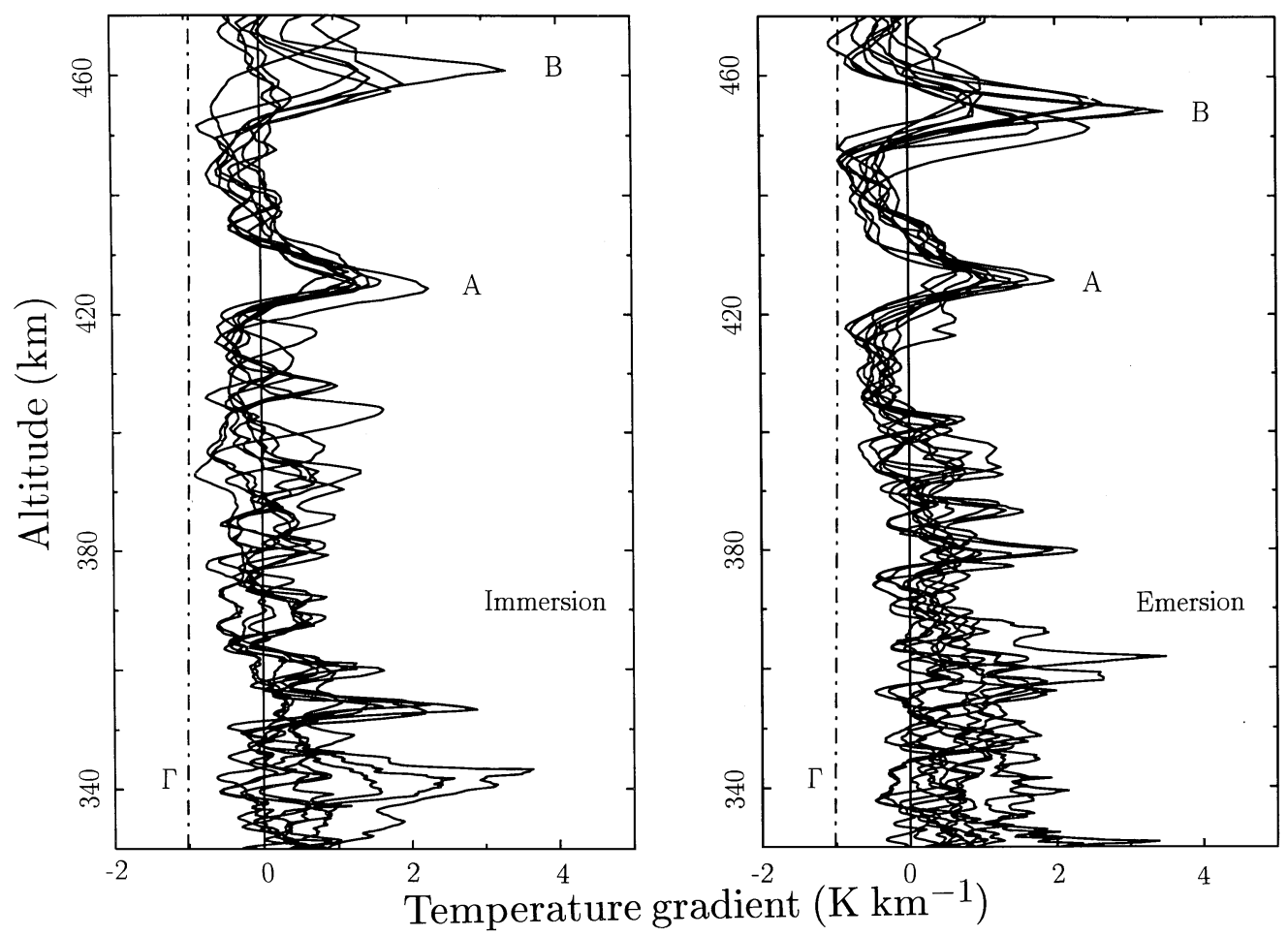

FIG. 9. Temperature gradient profiles at immersion (left) and emersion (right). The dash-dotted line indicates the adiabatic lapse rate $\Gamma$ obtained from Eq. (4). The general trend toward higher values of the gradient at low altitude (especially at emersion) is a spurious effect caused by the aerosol layer.

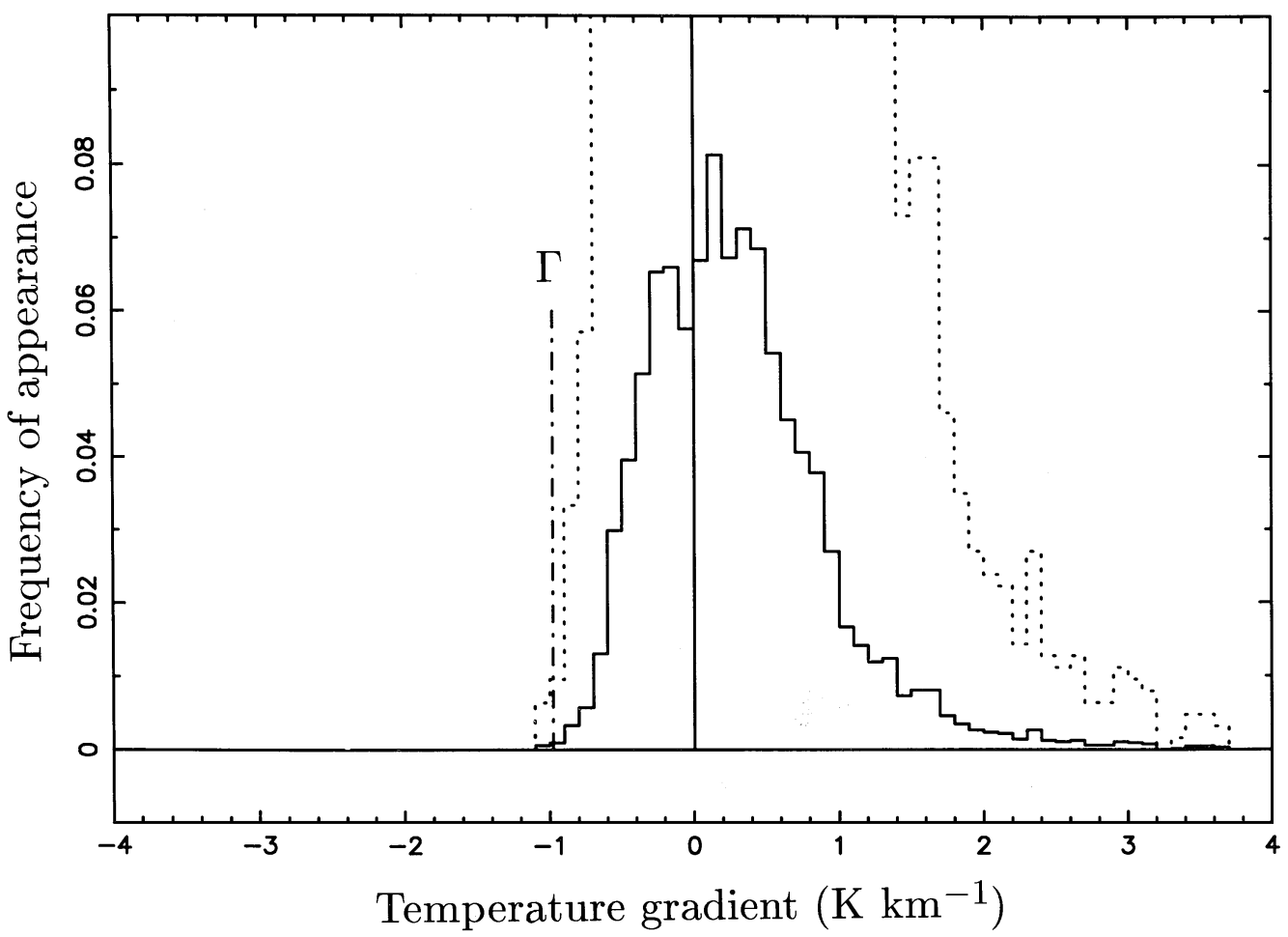

FIG. 10. Cumulative histogram of the temperature gradients $\partial T / \partial z$ bewteen 330 and $470 \mathrm{~km}$, for selected profiles (see text). Note the strong cutoff at the adiabatic lapse rate $\Gamma$. To show better the tails of the distribution on both sides, the histogram has been expanded vertically by a factor 10 and then plotted as a dotted line. Note the asymmetry of the histogram with respect to 0 , with higher values of $|\partial T / \partial z|$ reached on the positive side. 

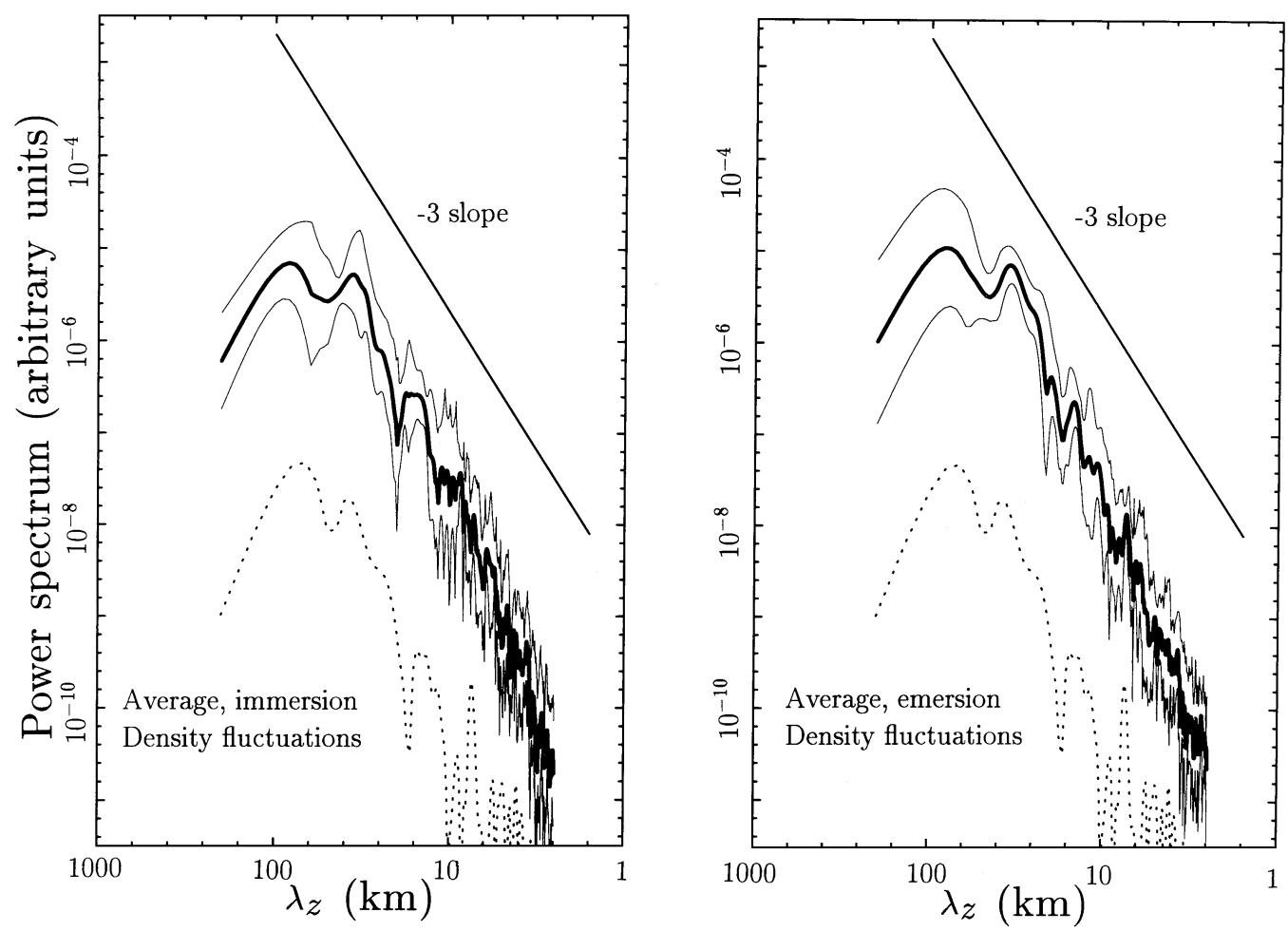

FIG. 11. Heavy lines: average of power spectra of density fluctuations, at immersion (left) and emersion (right). The thin lines indicate the \pm rms. deviations. At immersion, we used the profiles from Manley, Hertford, Greenwich, Langwedel, Essen, Pic du Midi (R channel), Ein Harod (R channel), and Wise. For emersion, we used the same stations plus the $\mathrm{R}$ channel of Vatican. The significance of the -3 slope is discussed in the text. The dotted line in each panel indicates the noise level; see text.

profiles have been considered only when the tangent of the angle between the stellar track and the local vertical is smaller than 10 . In other words, we assume that the aspect ratio of atmospheric features (horizontal to vertical typical lengths) is at least 10. This is a reasonable assumption, as seen in Section 8, where aspect ratios of $\sim 30$ or more are derived.

Practically, the spectra have been calculated for altitudes larger than $310 \mathrm{~km}$ for all the stations, except for Wise (altitudes larger than $343 \mathrm{~km}$ ) and Ein Harod (altitudes larger than $347 \mathrm{~km}$ ). The upper limit for all the profiles has been set at $500 \mathrm{~km}$ to avoid the exponential increase of the noise.

We have plotted in Fig. 11 an average of selected power spectra, choosing those data with sufficient signal-to-noise ratio and time resolution. Note that the rms dispersion around the average is rather small, indicating a robust power law behavior. The dotted lines indicate the noise level for these spectra. They have been obtained by generating synthetic lightcurves to which scintillation or photon noise (depending of the data) has been added. Note that the actual spectra dominate the noise by a factor $\sim 100$ to 1000 between wavelengths of $\sim 5$ and $50 \mathrm{~km}$. Thus, the derived spectra are largely dominated by fluctuations in Titan's atmosphere, not by ground-based noise. We have also drawn in this figure the -3 slope expected for saturated gravity wave power spectra (see the discussion in Section 9). The observed slope seems steeper $(\sim-4)$, in particular at high frequencies.
However, as we see now, the effects of both the stellar diameter and ray crossings can alter the original spectrum and steepen the slope.

\section{EFFECT OF RAY CROSSING AND STELLAR DIAMETER}

\subsection{Retrieval of the Vertical Profiles}

The question addressed here is the role of ray crossing in the retrieved vertical density or temperature profiles, in particular the asymmetries of the temperature gradients and the cutoff and the adiabatic lapse rate $\Gamma$, as shown in Figs. 9 and 10.

A priori, these results indicate that we do observe in Titan's stratosphere breaking of structures through convective instability. However, we will see that ray crossings can also mimic the same behavior in the spectral range considered here, i.e., for vertical wavelengths between $\sim 3$ and $50 \mathrm{~km}$.

We note first that ray crossing leads to a net loss of information, since we cannot distinguish in our data which path was followed by a given photon. The inversion procedure assumes that there is a one-to-one correspondence between the input impact parameter of the photon in the atmosphere and the output location of this photon when it arrives at the Earth. When this assumption breaks down, the retrieved profiles are distorted. Note, however, that this limitation is not inherent to the inversion 
procedure, and that the full information can be retrieved in the case of radio occultations, for which the frequency measurements allow ray crossing to be identified and interpreted (see, e.g., Hinson et al. 1998).

Let us consider a basically isothermal atmosphere of scale height $H$ and exponential density profile $n_{\exp }(z)$, with relative fluctuations $\epsilon(z)=\delta n(z) / n_{\exp }(z)=\delta v(z) / \nu_{\exp }(z)$. As shown in Appendix A (see also French and Lovelace 1983) a harmonic fluctuation with vertical extension $\lambda_{z}$ (i.e., with vertical wavenumber $m=2 \pi / \lambda_{z}$ ) will cause ray crossing if its relative amplitude is larger than

$$
\epsilon_{\mathrm{cr}}=\frac{1}{\left(1-\Phi_{0}\right)(m H)^{3 / 2}}
$$

where $\Phi_{0}$ is the Baum and Code flux associated with the smooth background atmosphere.

On the other hand, a given fluctuation will reach convective instability when the adiabatic lapse rate is reached, that is, for $\partial \epsilon / \partial z=(\gamma-1) /(\gamma H)$, i.e., for relative amplitudes larger than

$$
\epsilon_{\mathrm{ad}}=\frac{\gamma-1}{\gamma} \cdot \frac{1}{m H}
$$

where $\gamma$ is the adiabatic index.

Equations (5) and (6) show that

$$
\frac{\epsilon_{\mathrm{cr}}}{\epsilon_{\mathrm{ad}}}=\frac{1}{\sqrt{2 \pi}} \cdot \frac{1}{1-\Phi_{0}} \cdot \frac{\gamma}{\gamma-1} \cdot\left(\frac{\lambda_{z}}{H}\right)^{1 / 2} .
$$

In our case, $\gamma \sim 1.4$, and the most significant results are obtained for $\Phi_{0}$ lying between $\sim 0$ and 0.5. Thus, $\epsilon_{\mathrm{cr}} / \epsilon_{\mathrm{ad}} \sim \sqrt{\lambda_{z} / H}$.

This is quite unfortunate in the present study, since the vertical scales that we are probing (roughly in the range 3-50 km) yield values of $\epsilon_{\mathrm{cr}} / \epsilon_{\mathrm{ad}}$ of order unity. In other words, the fluctuations are reaching the convective instability at about the same time as they cause ray crossing. Note that this ambiguity is the result of a fortuitous combination of the numerical values of $\gamma$, the scale height $H$, and the typical vertical extensions $\lambda_{z}$ considered here, and is not intrinsic to the inversion method.

There is a further coincidence that complicates the interpretation of diagrams such as Fig. 9. Ray crossing occurs when the photons encounter a local relative increase in density with height, to counteract the defocusing effect caused by the general exponential decay of density with altitude. This corresponds to a local decrease in temperature with height, i.e., a local negative gradient of temperature. In other words, ray crossing occurs at the same place where convective instability is taking place. This is well visible in the left panel of Fig. 15B or the left panel of Fig. 16A.

As shown by French and Lovelace (1983) (and see also Figs. 15, 16), ray crossing induces a damping of the retrieved structures after the inversion has been performed, which can ex- plain the asymmetry of Fig. 9. However, Eq. (7) shows that ray crossing is less likely for the larger vertical structures, which should therefore be better retrieved.

This is illustrated in Fig. 12, where we model the two inversion layers A and B of Fig. 9. The solid line in the left panel is the initial temperature gradient profile, and the dash-dotted line is the retrieved profile after inversion. Since no ray crossing is induced by this structure, and also because it is much larger than the stellar diameter at that level (about $13 \mathrm{~km}$ ), the retrieved profile agrees very well with the original one. If the amplitude of the temperature fluctuation is multiplied by 2 , however, the negative side of the profile induces ray crossings, and is then seriously damped after the inversion (dotted line). Although distorted, the positive side of the profile is better retrieved, since it does not induce ray crossing.

We have performed various tests on this particular feature, varying the amplitude and the asymmetry of the gradients to fit the observations. We could not find any satisfactory way to reproduce the observed profile (right panel of Fig. 12) by ray crossing only, except for quite exotic models where the temperature has a strong local superadiabatic lapse rate of several times the values of $\Gamma=-1 \mathrm{~K} \mathrm{~km}^{-1}$. Such a highly unstable layer is unlikely to be maintained.

Thus, the general behavior of the temperature profiles near layers A and B is likely to correspond to real inversion layers, separated by a convectively unstable region. This may be not the case for smaller structures, as explained below.

\subsection{Retrieval of the Vertical Spectra}

The spectra shown in Fig. 11 can be altered both by the stellar diameter and by ray crossing. These two effects tend to smooth out the high frequencies, so that the spectra presented here must be taken with some care.

To study these effects, we have generated in a direct way occultation lightcurves from a model atmosphere with some prescribed structure. We use a method of ray tracing, along the lines presented in Section 3.2. Only geometrical optics is considered (no diffraction), while the smoothing by the stellar diameter is taken into account in most of the profiles. It is the dominant source of smoothing since the stellar diameter at Titan $(18 \mathrm{~km})$ is larger than the Fresnel scale $(\sim 1 \mathrm{~km})$. Once generated, the lightcurve is inverted with our usual code.

Figure 13 shows the transfer function for the stellar diameter. Temperature fluctuations with a flat power spectrum are first superimposed on an isothermal profile. A lightcurve is then generated, and the smoothing by the stellar diameter is applied. In the final step, an inversion is performed and the resulting power spectrum of the retrieved temperature profile is plotted as a thick line. One first notes that at low frequency there is a slight excess of power in the retrieved spectrum, due to the fact that the inversion does not provide a strictly isothermal profile, but rather adds some low-frequency features to the profile. Second, one can see the gradual tapering of the spectrum at higher frequency due to the stellar diameter. This induces an artificial slope of about -2 


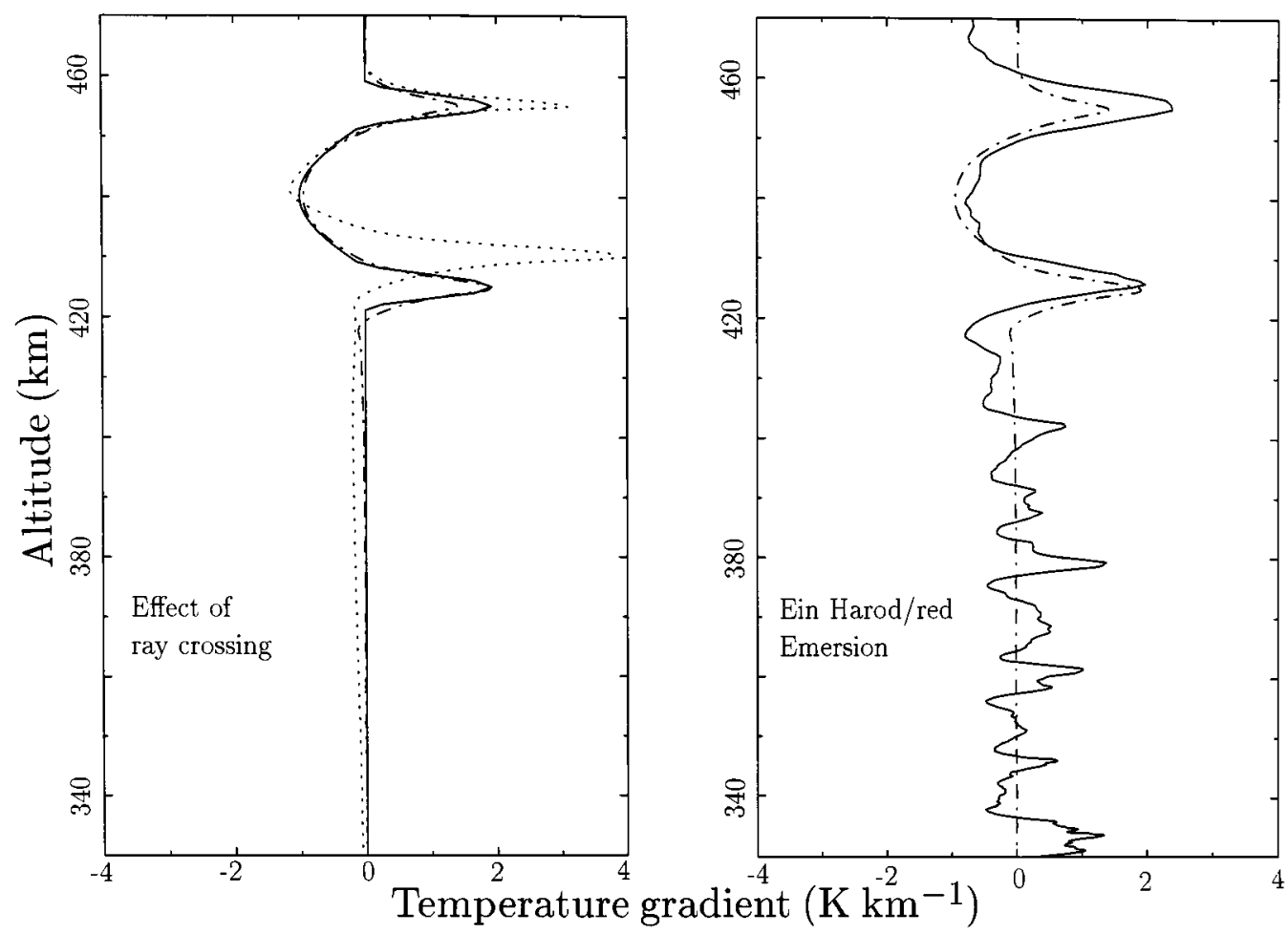

FIG. 12. Model for the double-inversion layer observed at Ein Harod emersion (red channel). Left panel: The input model is shown as a solid line. The retrieved gradient profile (after inversion, taking into account the stellar diameter) is plotted as a dash-dotted line; note that it is almost undistinguishable from the original profile. To illustrate the effect of ray crossing, the input model has been multiplied by 2 and then inverted (dotted line). Note that the retrieved dotted profile is not multiplied by 2 . Rather, the negative gradients are bounded by approximately $-1 \mathrm{~K} \mathrm{~km}^{-1}$, while the positive spikes are distorted. Thus, ray crossing can simulate the saturation of an atmospheric wave by the adiabatic lapse rate. Right panel: Comparison of the retrieved profile (dash-dotted line, already shown in the left panel) with the temperature gradients of Ein Harod. See the text for details.

to the resulting power spectrum. There is finally a sharp drop in power for wavelengths smaller than $\sim 3 \mathrm{~km}$. This drop is caused by the stellar diameter and, to a lesser extent, by the fact ray crossing starts to play an important role as higher frequencies are encountered.

These two effects are now examined in more detail, considering original spectra with slope $-2,-3$, and -4 . Figure 14 shows for instance that the stellar diameter of the star alone can steepen an original -2 spectrum (Fig. 14A, left) into a $\sim-3$ spectrum (Fig. 14A, right). Note, however, that an original -3 spectrum is little affected by the stellar diameter (Fig. 14B, left) and is also little affected by severe ray crossing (Fig. 14B, right), except for some loss of signal at higher frequencies, leading to a slope closer to -4 than to -3 .

The effect of ray crossing is complex in the details, but it tends to steepen a shallow spectrum toward a -3 spectrum. This is because the required amplitude $\epsilon_{\mathrm{cr}}$ for a fluctuation to yield ray crossing is proportional to $m^{-3 / 2}$ [Eq. (5)]. Beyond ray crossing, the retrieved fluctuation is severely damped. Consequently, if ray crossings occur at all scales, the retrieved power spectrum is proportional to $\epsilon_{\mathrm{cr}}^{2} \propto \mathrm{m}^{-3}$, even if the original spectrum is shallower than that.

This effect is illustrated in Fig. 15. Temperature fluctuations (and therefore density fluctuations) are first generated with a flat spectrum. The thin line in the left panel of Fig. 15A shows the temperature gradient of that profile. This original profile is used to generate a lightcurve, taking into account the smoothing by the stellar diameter. This lightcurve is then inverted, yielding the thick line in the Fig. 15A, left. One can see that ray crossing has very seriously damped the original profile. Also, the Fig. 15A, right, shows that the originally flat spectrum has been transformed in a much steeper, roughly -3 , power spectrum.

The same effect is observed in the Fig. 15B. There, an original temperature profile with a -2 spectrum is generated. Note that ray crossing causes a tapering of the negative temperature gradients after inversion, as anticipated in the previous subsection. On the other hand, note that the positive gradients are well reproduced after inversion. Also, the absolute altitude of each feature is well retrieved. Figure 16 shows further versions of ray crossing effects, with steeper original spectra ( -3 and -4 slopes).

Simulations of both Figs. 15 and 16 should be compared with actual profiles and spectra, as shown in Fig. 17 for Pic du Midi (or in Fig. 11 for an averaged version). The observed power spectra generally exhibit a general -3 slope at low frequencies, followed by a $\sim-4$ slope at higher frequencies.

This comparison should make us rather cautious in interpreting the vertical spectra obtained here, even though some quantitative results can be derived from Figs. 15 and 16. In these 


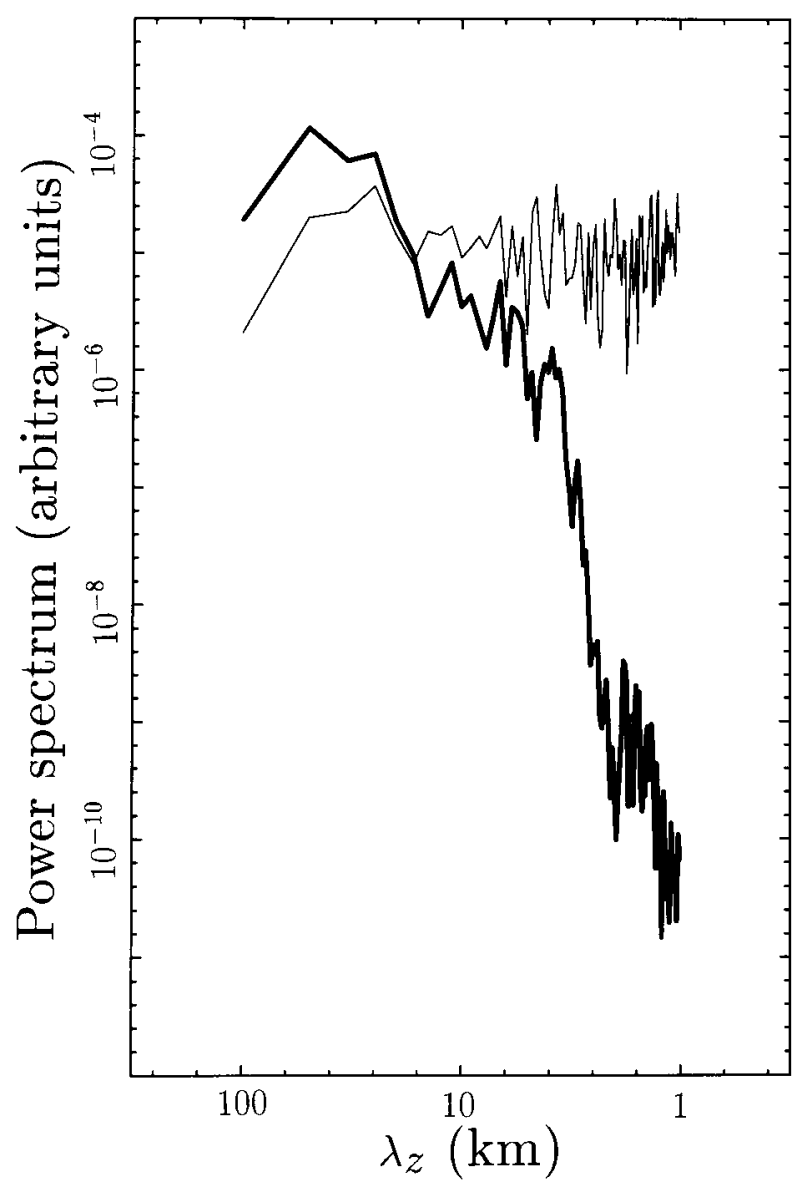

FIG. 13. An originally flat power spectrum of temperature fluctuations was first generated, and is shown here as a thin line. A synthetic lightcurve was then generated using the corresponding density profile, and it was smoothed by the stellar diameter before being inverted. The resulting power spectrum of the retrieved temperature profile is plotted as a thick line. See text for comments.

figures, the synthetic profiles have an amplitude that is chosen so as to roughly reproduce the observed typical amplitudes, e.g., at Pic du Midi (Fig. 17).

This shows for instance that an original flat spectrum is not a good model for Titan's atmosphere. In effect, the amplitude of the original profile of Fig. 15A (left) results in temperature gradients of several times the adiabatic lapse rate $\Gamma$. Such a highly unstable atmosphere is unlikely to be maintained, even by breaking gravity waves. Also, a -4 original spectrum yields too steep a retrieved spectrum (Fig. 16B) and contains too little energy at high frequency when compared with real data (Fig. 17).

The intermediate cases ( -2 and -3 spectra) are both consistent with the observations. The observed spectra could be explained either by -2 spectra steepened by stellar diameter smoothing and ray crossings, or by a pure -3 original spectrum with no ray crossing (and, thus, with real adiabatic lapse rate cutoff), or by a mixture of the two situations.

Note, however, that there is no special reason why the cutoff of temperature gradients should be exactly at $\Gamma$ if it were caused by ray crossing only. Thus the profiles shown in Fig. 9 are probably close to reality, the features being close to the convective instability limit and the spectrum being close to a -3 power law.

\section{HORIZONTAL LAYERING}

\subsection{Global Trends along the Limb}

We have been concerned up to now with vertical structure of individual profiles. Because of the geographical coverage available from various stations (Fig. 1), the horizontal structure of the atmosphere can be probed as well. In particular, we would like to evaluate the horizontal coherence of some features. To do so, we use the profiles of temperature gradient, $\partial T / \partial z$. They are better suited than the temperature profiles $T(z)$ since they are less affected by general linear trends, while preserving the information on the local temperature fluctuations. Figure 18 shows the $\partial T / \partial z$ profiles at immersion and emersion on a large scale. Each profile has been shifted horizontally by the distance, along the limb of Titan, to the northernmost station (Manley). Figure 19 shows more local features, as observed from the closely packed European stations. The two panels in this figure show subtle variations in the small structures that are discussed below.

To study possible trends with latitude (due for instance to seasonal effects), we have plotted the profiles according to latitude, not distance, in Fig. 20. As noted in the caption to Fig. 18, the Catania profiles appear somewhat smoothed out due to the larger integration time step used there. Consequently, layer $\mathrm{A}$ at that station does not appear as sharp as in the other stations. A close examination of Fig. 20 shows that layer A is a global feature visible from latitude $\Phi \sim-45^{\circ}$ to $\Phi \sim+20^{\circ}$. Layer B, on the other side, is readily visible only at latitudes $\Phi$ north of $\sim-20^{\circ}$.

It is interesting to note that the two layers $A$ and $B$ are well correlated between the northern immersion stations and the southern emersion stations. This may be a priori surprising as the corresponding points in Titan are separated by almost one Titan diameter (Fig. 1). A closeup view of this correlation is shown in Fig. 20B. This indicates that layers A and B are zonal features that extend around the whole satellite at a given latitude.

\subsection{Cross-Correlation of Fine Features}

To have a more quantitative description of the horizontal layering of the atmosphere, we have evaluated the cross-correlation coefficient of the various profiles in a given altitude interval. Let $\operatorname{gr}(z)=\partial T / \partial z$ be the temperature gradient at altitude $z$ derived from a given station. This profile is first resampled by linear interpolation with a regular and fixed vertical step of $0.2 \mathrm{~km}$. Let $\left(z_{i}, g r_{i}\right)$ and $\left(z_{i}^{\prime}, g r_{i}^{\prime}\right)$ be two such profiles derived for two stations. Then, the cross-correlation ${ }^{5}$ coefficient $C C$ is defined as

$$
\begin{aligned}
C C= & \sum_{i}\left(g r_{i}-\overline{g r}\right) \cdot\left(g r_{i}^{\prime}-\overline{g r^{\prime}}\right) / \\
& \sqrt{\sum_{i}\left(g r_{i}-\overline{g r}\right)^{2} \cdot \sum_{i}\left(g r_{i}^{\prime}-\overline{g r^{\prime}}\right)^{2}},
\end{aligned}
$$

\footnotetext{
5 Just called "correlation" hereafter.
} 

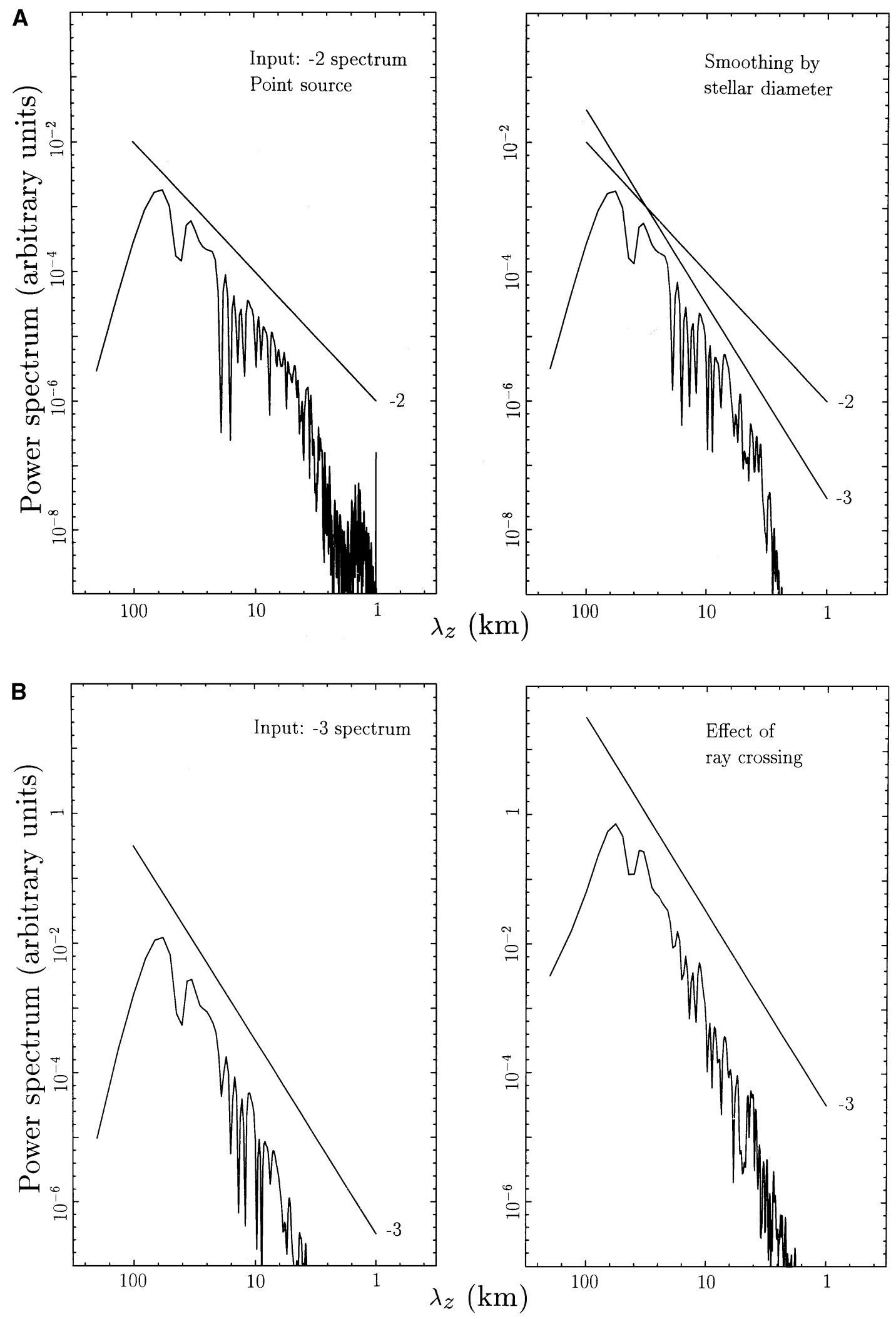

FIG. 14. A synthetic occultation lightcurve is generated with some vertical density fluctuations superimposed to an isothermal atmosphere, and then inverted. (A, left) The original power spectrum of density fluctuations is a power law of exponent -2 . The spectrum of temperature fluctuations, after inversion, is satisfactorily retrieved in this panel, assuming that the star is a point source. Note, however, the steepening of the spectrum at the right end, due to ray crossing. (A, right) Before inversion, the synthetic lightcurve has been smoothed by the stellar diameter projected at Titan (taking into a account the vertical contraction of the image at lower altitudes). The retrieved spectrum is then steepened to a slope of approximately -3. (B, left) Same as (A, right), panel i.e., taking into account the stellar diameter, except that the original spectrum has a slope of -3 . (B, right) Same as $(B$, left), except that the fluctuations have been multipled by a factor of 10 , which induces ray crossings. See text for discussion. 

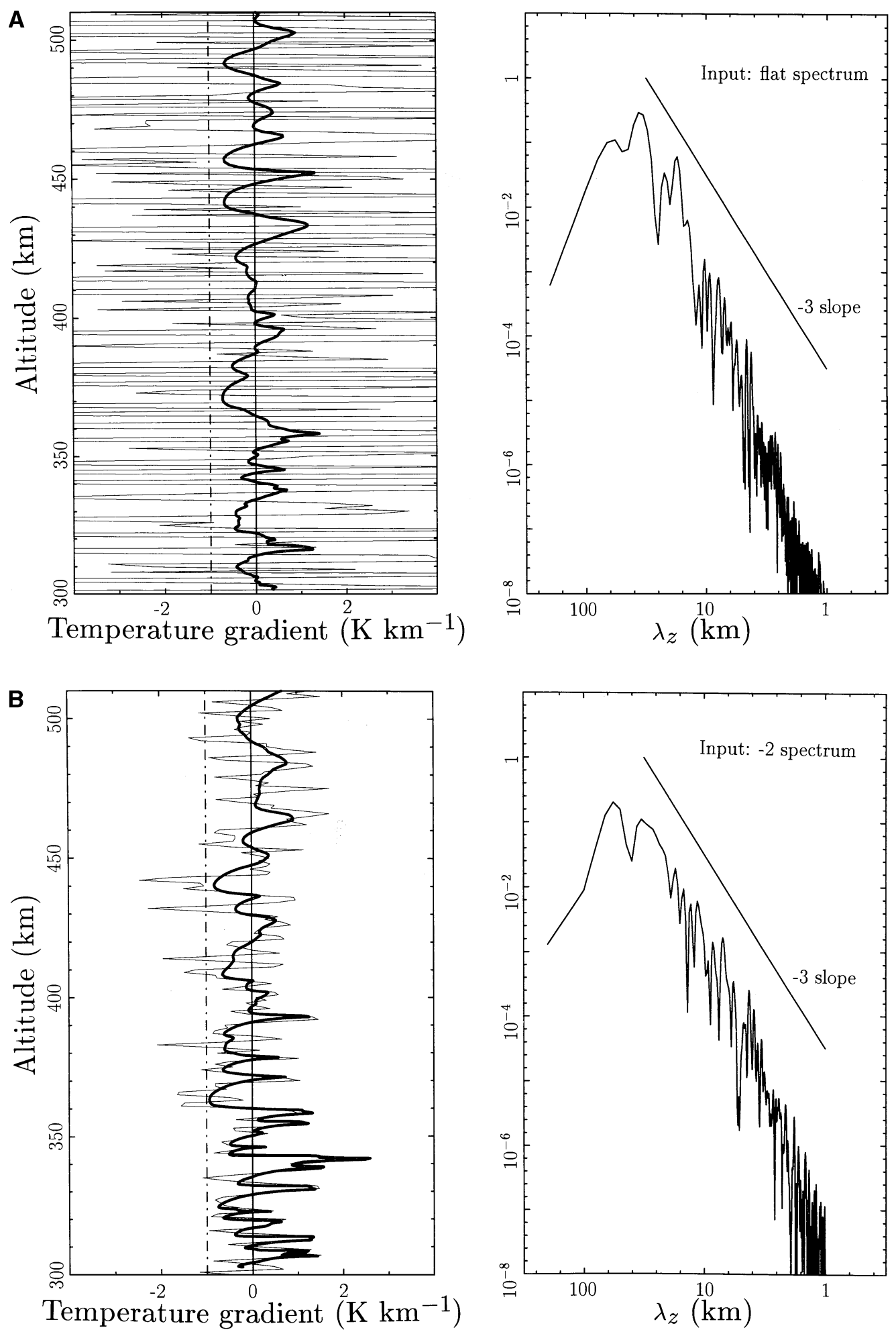

FIG. 15. Left panels: The thin lines are synthetic temperature gradient profiles, and the thick lines are the retrieved profiles after inversion of the corresponding lightcurve (the stellar diameter is taken into account). The dot-dashed line shows the adiabatic lapse rate $\Gamma$. Right panels: Power spectra of the retrieved temperature profiles found in the left panels. (A): The original temperature power spectrum is flat. (B): The original power spectrum has a slope of -2 . Note that the smoothing by the stellar diameter, substantial at higher altitudes in the (B, left), diminishes at lower altitudes as the apparent stellar diameter shrinks. 

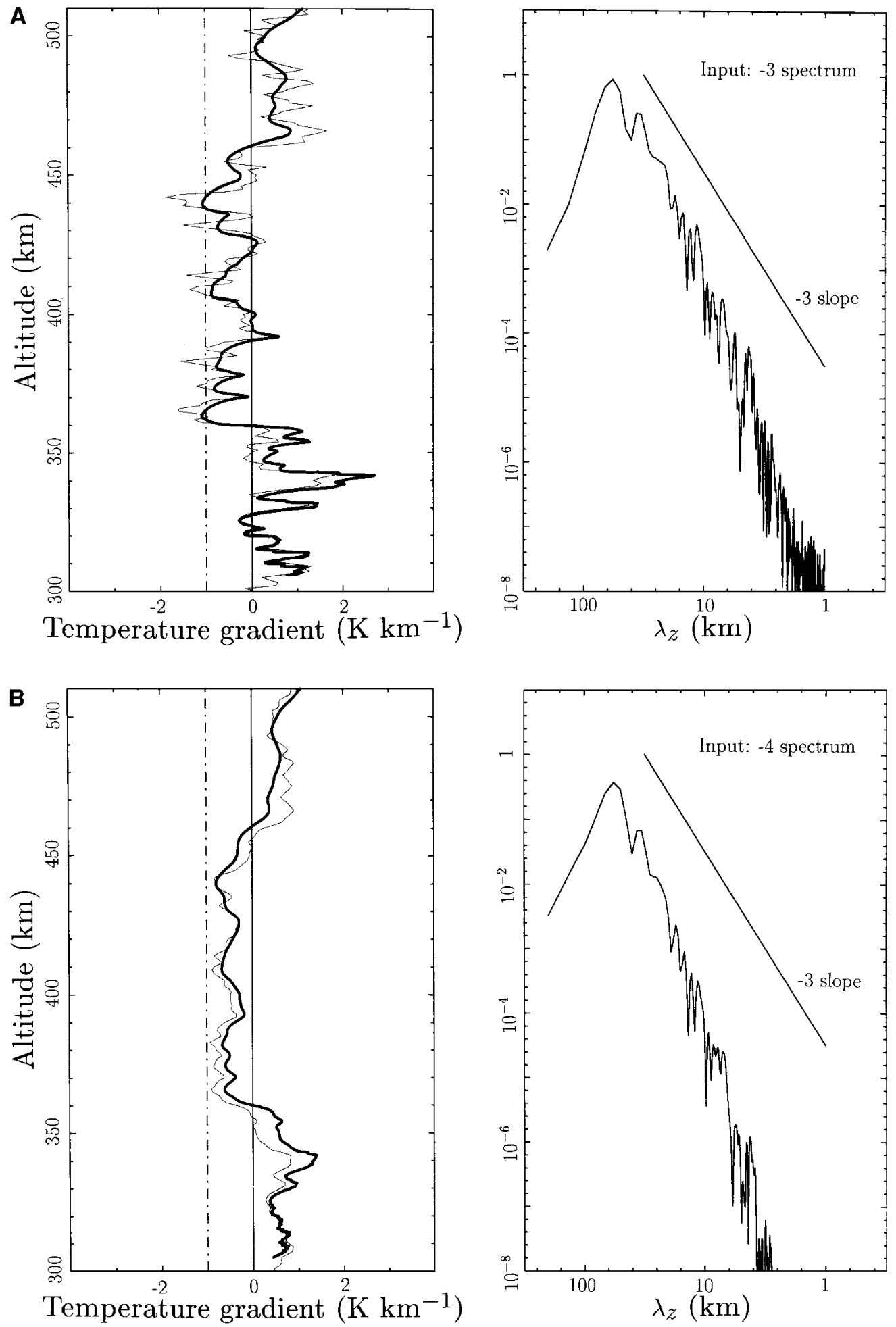

FIG. 16. Same as the previous figure, with a -3 spectrum (A) and a -4 spectrum (B).

where the bars denote the average value over the interval considered.To account for possible small misalignments of the structures, we have allowed vertical shifts of one of the profiles with respect to the other, by increments $\Delta z$ of $1 \mathrm{~km}$ in the limit $\Delta z= \pm 10 \mathrm{~km}$ around the zero nominal value. We have then re- tained the maximum value of $C C$ obtained in this process, and used it in the plots shown in Fig. 21.

One can see that the correlation corresponding to Interval 1 (bracketing layer A between 410 and $440 \mathrm{~km}$, see Fig. 19) remains high, namely, greater than $80 \%$, even at large distances. 

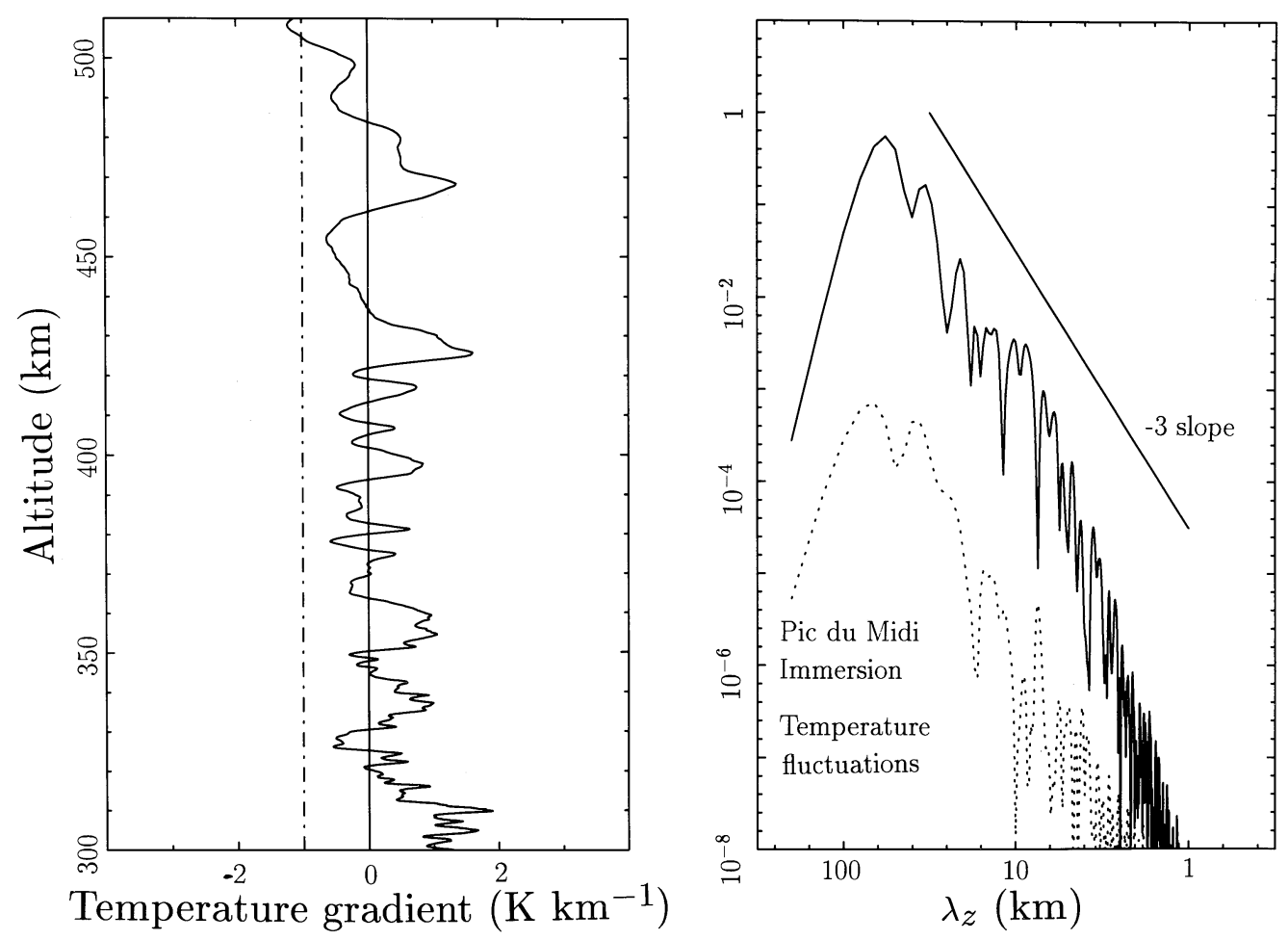

FIG. 17. Left panel: Temperature gradient profile obtained at Pic du Midi, immersion. As in Figs. 15 and 16, note the increasing spatial resolution as deeper layers are probed. Right panel: Power spectrum of the temperature fluctuations (between altitudes of 310 and $500 \mathrm{~km}$ ) corresponding to the left panel. See text for details. As in Fig. 11, the dotted line indicates the noise level.

This shows that the structure of layer A is essentially the same in all profiles, as was expected from Fig. 20. The correlation corresponding to Interval 2 (see Fig. 19) exhibits a drastically different behavior, with a clear decrease with distance, and a drop of $50 \%$ for a distance of $250 \mathrm{~km}$ along the limb. On the other hand, the autocorrelation of Interval 2 drops to $50 \%$ for a vertical displacement of about $1.8 \mathrm{~km}$, indicating an aspect ratio $\rho$ of the features (ratio of horizontal to vertical characteristic lengths) of about 140 in that interval.

\subsection{Correlations of the Israel Lightcurves}

A more detailed study of the horizontal structure of the atmosphere is possible using the lightcurves obtained at the Israeli stations, Ein Harod and Wise (see Fig. 2). The corresponding stellar tracks have the advantage of being relatively clear of aerosols (see Fig. 4 of H93), so that the stellar flux was detected with a good signal-to-noise ratio all around the southern limb of Titan at these stations (Figs. 4 and 25).

Furthermore, the Ein Harod detector could record simultaneously in two channels, at 0.45 and $0.80 \mu \mathrm{m}$. Because the refractivity of the gas is decreasing with wavelength, the "blue" stellar image at a given moment is slightly higher in the atmosphere than the "red" stellar image. Assuming a pure $N_{2}$ atmosphere, and a smooth temperature profile as calculated by Yelle (1991) (see Section 3.2), one can calculate the trajectory of the stel- lar image in the atmosphere. The result is shown in Fig. 22, where the altitude $z$ of the stellar image in Titan's atmosphere is plotted versus the distance $x$ traveled along the limb. Note the large difference (more than two orders of magnitude) between the vertical and horizontal scales. Actually, the stellar images are following essentially horizontal tracks in the atmosphere, as is evident from Fig. 2.

As shown in Appendix A, the ratio $\Phi_{\mathrm{sm}} / \Phi$ of the smooth background stellar flux to the actual observed flux depends on both the altitude $z$ and the distance $x$ along the limb [Eq. (A4)]. An example of the ratio $\Phi_{\mathrm{sm}} / \Phi$ is shown in Fig. 25 as a function of $x$ for the Wise data. For each lightcurve, this ratio was resampled according to $z$ only, and then compared with the ratio $\Phi_{\mathrm{sm}} / \Phi$ obtained from another lightcurve. For a given altitude $z$, let $\delta x(z)$ be the distance along the limb of the two stellar images observed by the two experiments. For instance, for the two channels of the Ein Harod detector, the distance $\delta x(z)$ ranges from about $1 \mathrm{~km}$ around $z=500 \mathrm{~km}$ to about $60 \mathrm{~km}$ around $z=300 \mathrm{~km}$ (Fig. 22).

We can then calculate the cross-correlation $C C(z)$ of the two ratios $\Phi_{\mathrm{sm}} / \Phi$ over a finite vertical interval $z \pm \Delta z / 2$. Typically, $\Delta z=10 \mathrm{~km}$, but we may choose larger correlation intervals higher in the atmosphere and smaller ones lower down. We can finally calculate the autocorrelation of $\Phi_{\mathrm{sm}} / \Phi$, and determine for which displacement $\delta z$ the autocorrelation reaches the value $C C(z)$. Then, the typical aspect ratio $\rho$ of the structures detected 

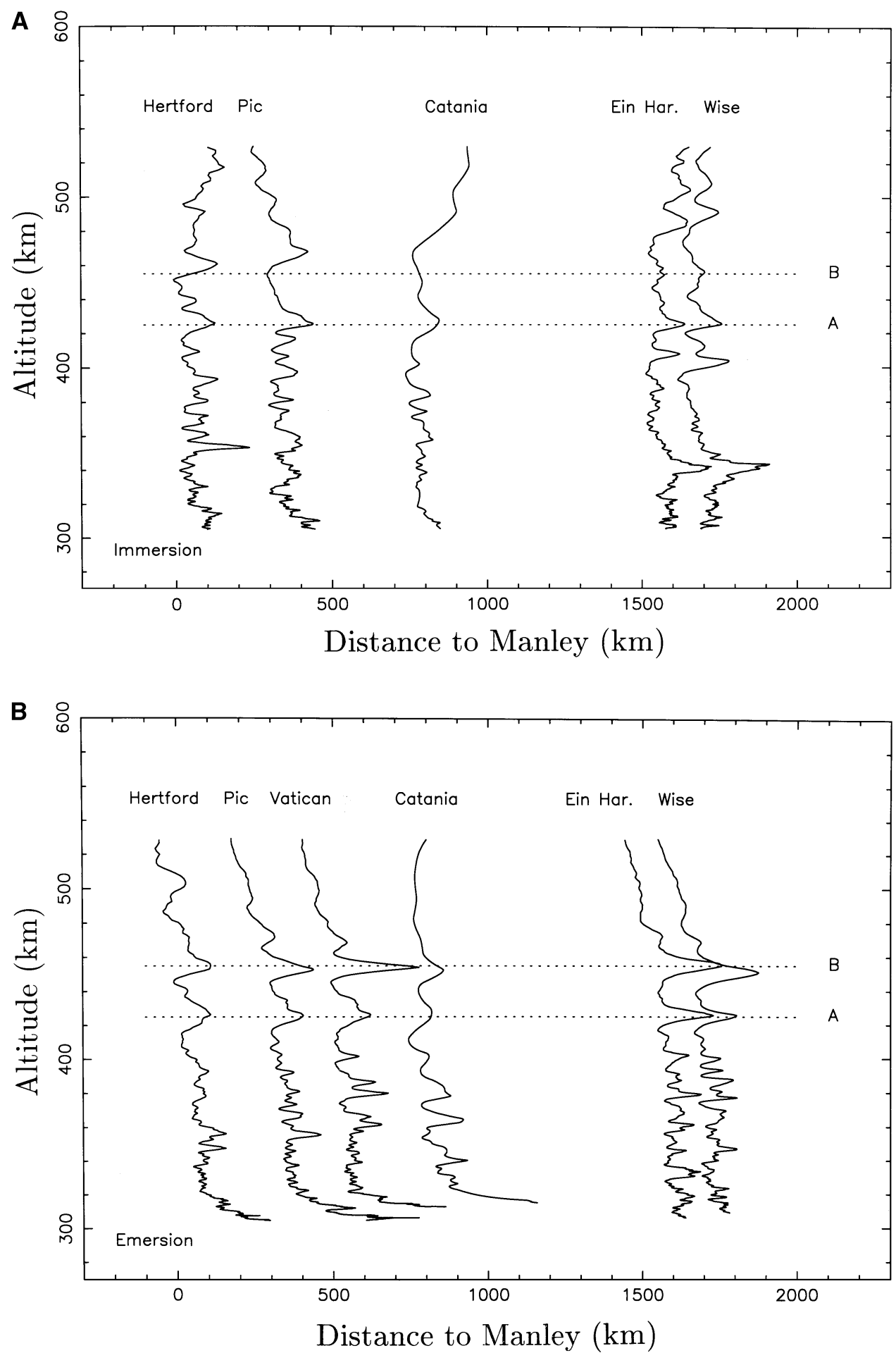

FIG. 18. (A) Temperature gradients profiles obtained at chosen stations during immersion. Each profile has been shifted horizontally according to its distance (along the limb) to the Manley station. (B) The same for emersion profiles. The prominent and ubiquitous inversion layer A can be seen in both panels. Note: The Catania profile appears smoother than the other profiles because of the larger integration time step, $1 \mathrm{~s}$, used at that station (see Table I). 

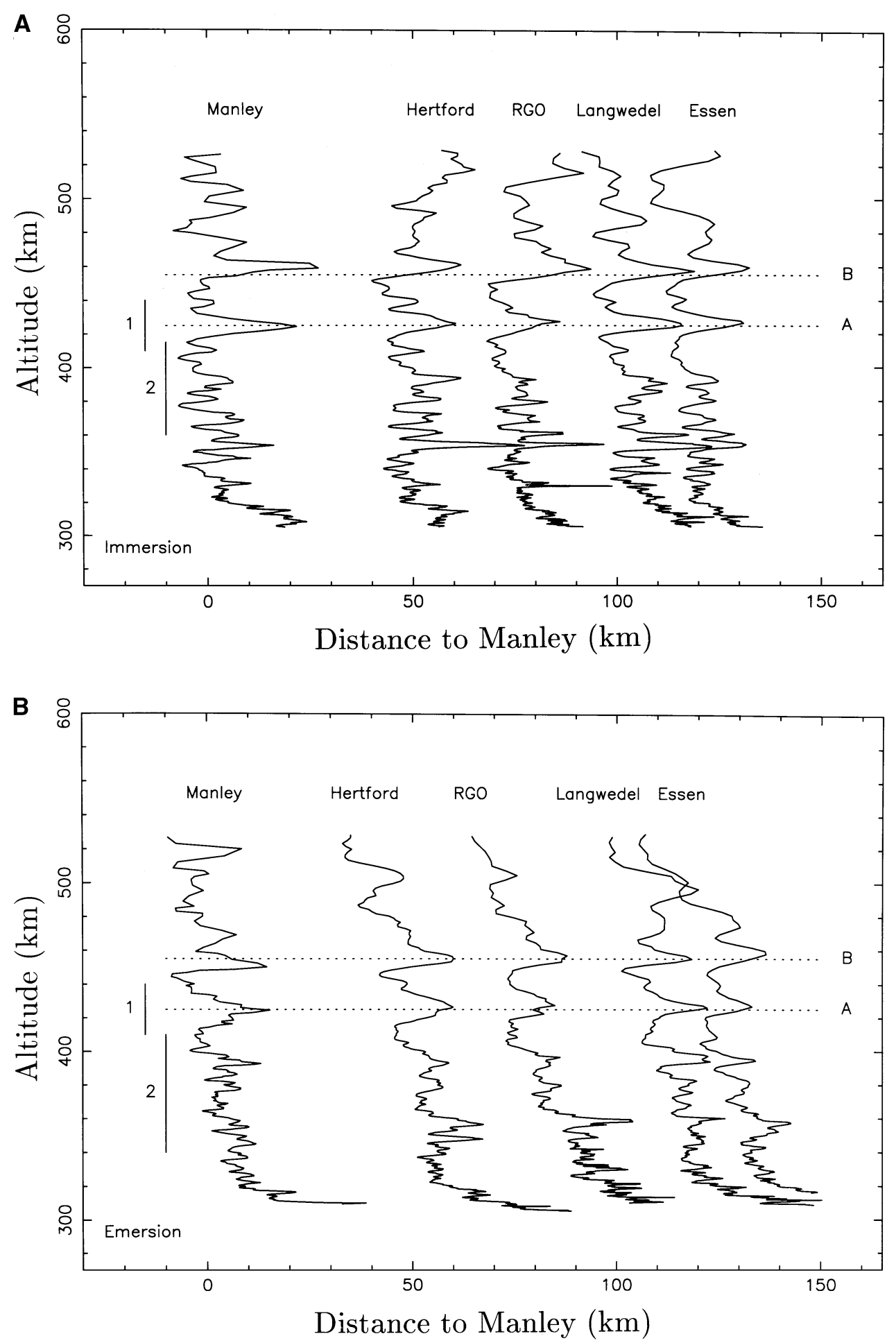

FIG . 19. Same as Fig. 18, but on a more local scale to show the correlations between the European profiles. (A) Immersion profiles. The vertical lines labeled 1 and 2 show the altitude intervals used for cross-correlation calculations; see the text and Fig. 21. (B) Emersion profiles. 

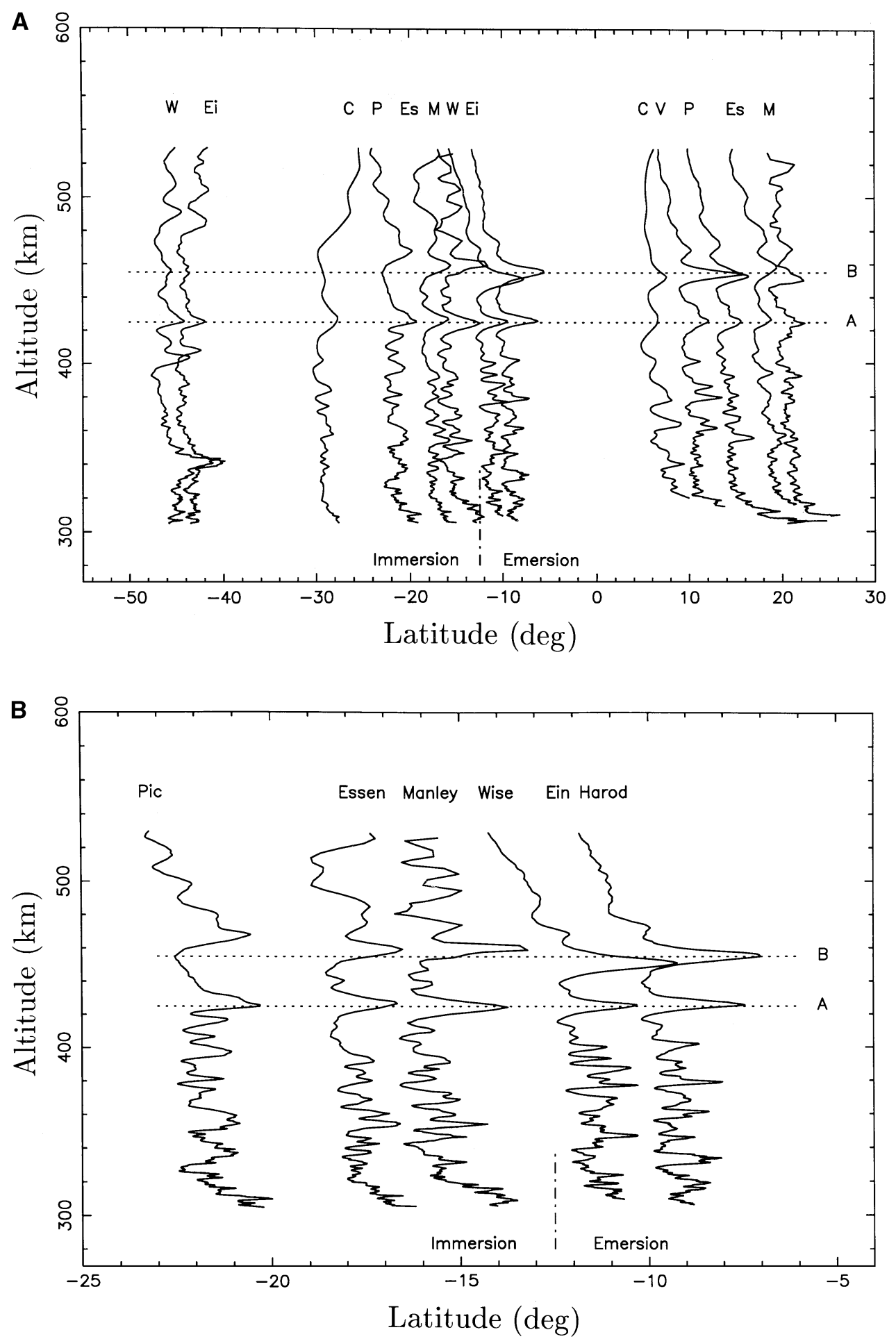

FIG. 20. Same as Fig. 18, but the profiles have now been shifted according to the titanocentric latitude. (A) General view showing all the latitude coverage obtained during the occultation. (B) Expanded view showing some European profiles at immersion, compared with the Israel profiles at emersion. Note that although the two sets of profiles probe opposite limbs of the satellite (see Fig. 1), there is a strong correlation of the profiles of layers A and B. 


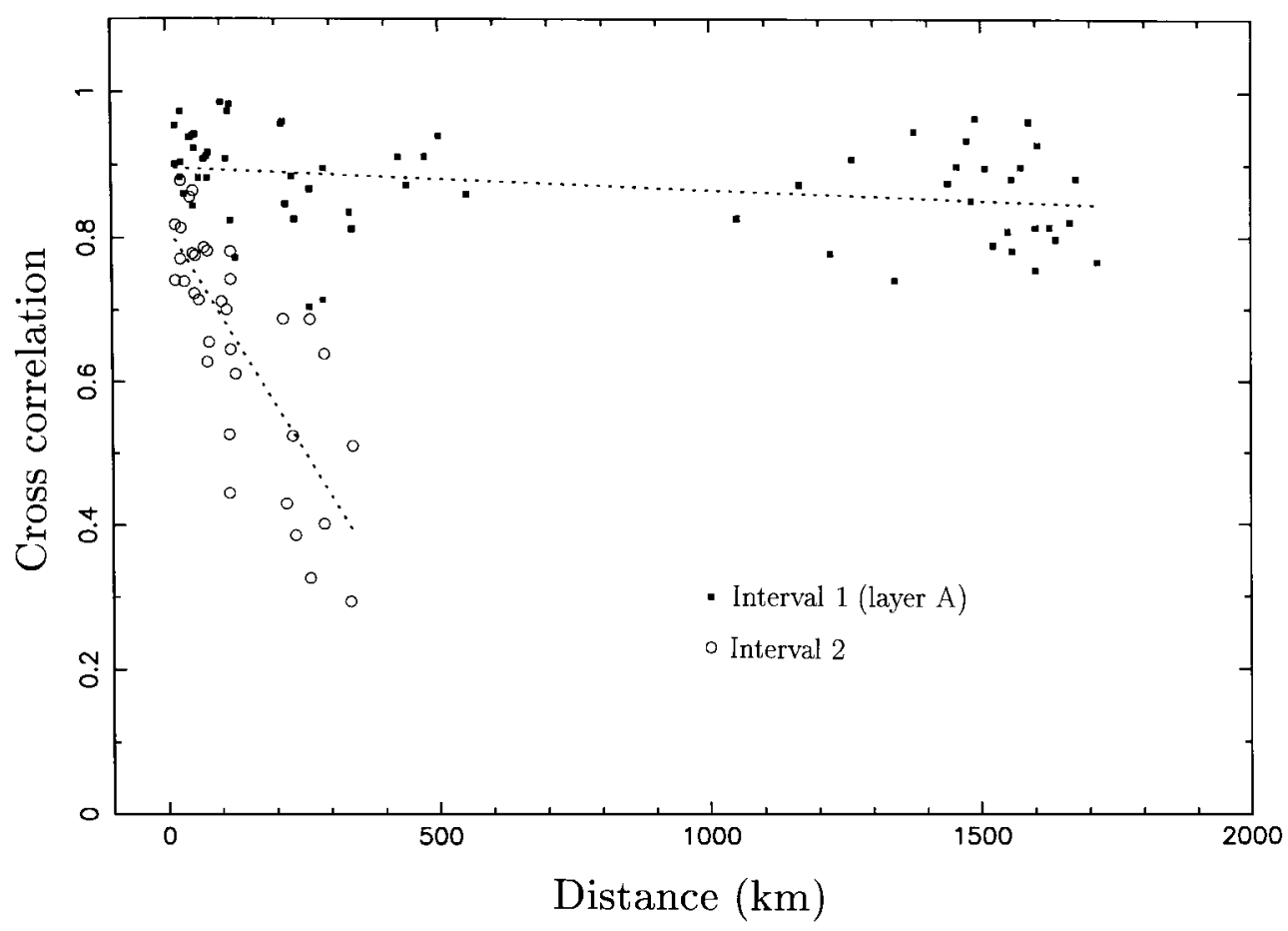

FIG . 21. Filled squares: Cross-correlation coefficients $C C$ (see text) corresponding to interval 1 (see Fig. 19), versus the distance along the limb of Titan. Open circles: The same for Interval 2. Both immersion and emersion profiles have been used to obtained these $C C$ values. The dotted lines show the linear regressions for each set of points.

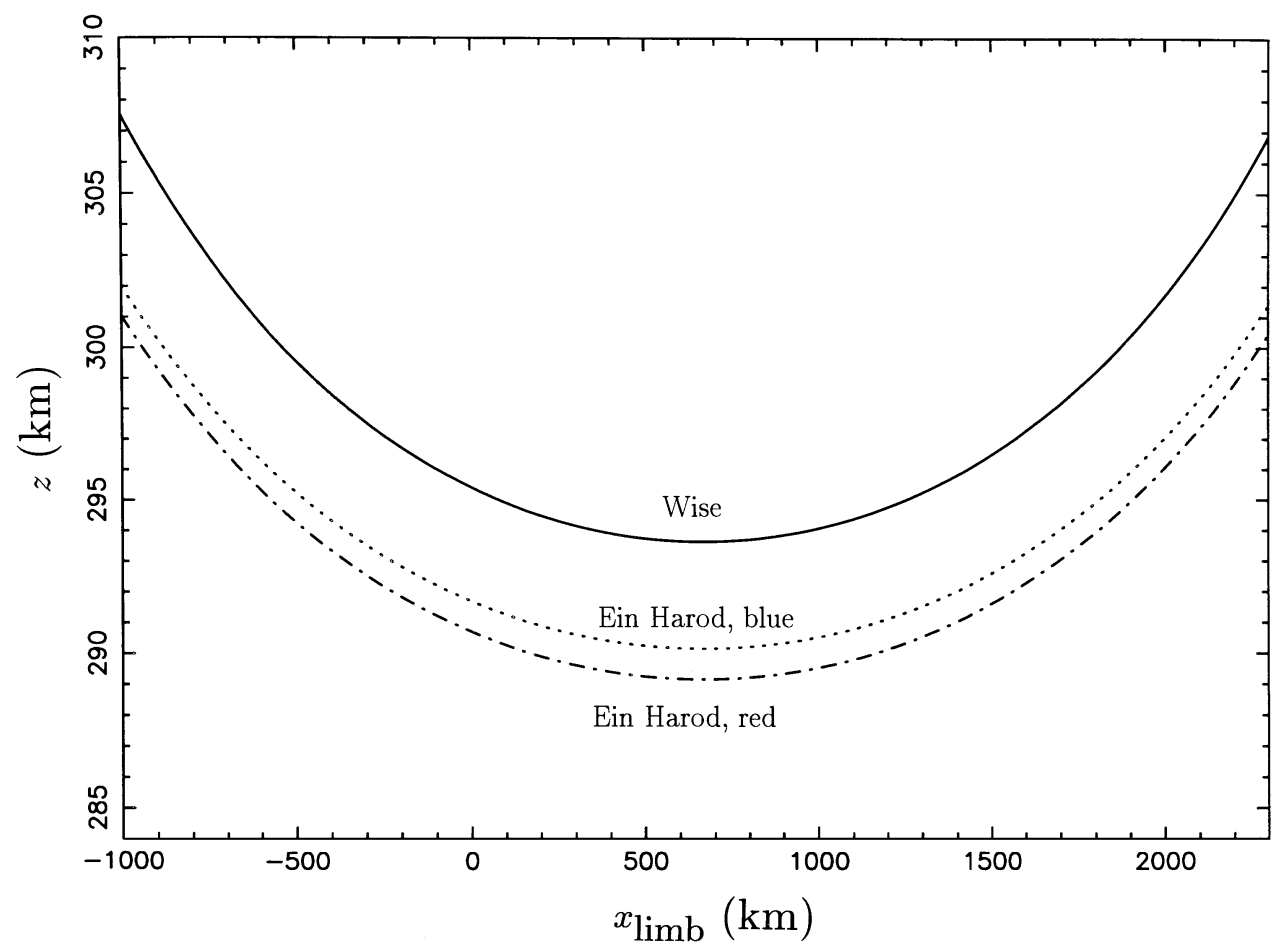

FIG. 22. Altitude $z$ of the stellar image observed from different stations, as a function of the distance $x$ traveled by the image along the southern limb of Titan. The origin of $x$ has been arbitrarily chosen so as to correspond to the intersection of the stellar image track with the north-south axis centered on Titan (corresponding to $\xi=0$ in Fig. 1). 


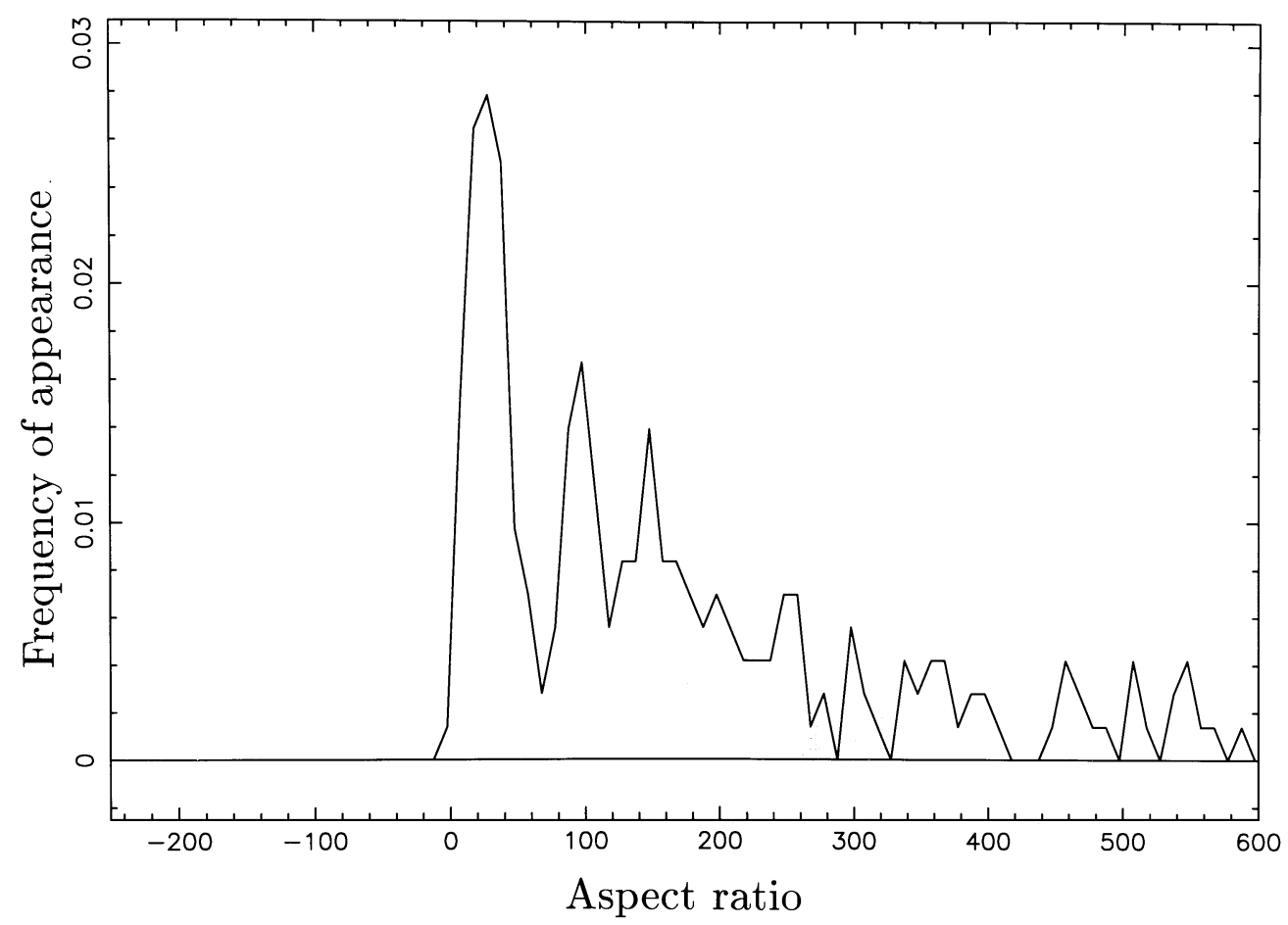

FIG. 23. Histogram of the aspect ratios of the atmospheric features, derived from the Ein Harod and Wise lightcurves. This histogram combines all the aspect ratios derived from comparing (i) the Ein Harod red and blue channels between themselves, (ii) the Ein Harod red channel and Wise lightcurve, and (iii) the Ein Harod blue channel and Wise lightcurve (see the text).

in the interval $z \pm \Delta z / 2$ is

$$
\rho=\left|\frac{\delta x}{\delta z}\right|
$$

A histogram of $\rho$ is displayed in Fig. 23, where we have compared the two channels of the Ein Harod detector between themselves and also with the Wise profile. Note the strong peak at $\rho \sim 30$, with a FWHM of $\sim 30$. This shows that we detect many horizontal features in the atmosphere with typical aspect ratios between $\sim 15$ and 45 . Note also the significant tail in the distribution of $\rho$, extending between $\sim 100$ and $\sim 250$, indicating that we do detect structures with aspect ratios up to 200 or even more. Remember from the previous subsection that the aspect ratio of the structures observed in Interval 2 of Fig. 19 was about 140.

Finally, for each vertical interval of correlation, we have determined the small vertical displacement $z_{\text {shift }}$ of one data set with respect to the other, necessary to maximize the correlation between the two data sets. The value of $z_{\text {shift }} / \delta x$ is thus a measure of the inclination of the features with respect to the local horizontal direction. Figure 24 shows a histogram of these inclinations obtained from the same data as before (Ein Harod and Wise). As expected, we observe a narrow spike around zero, with a dispersion of about $\pm 1.5^{\circ}\left(\sim \pm 2.5 \times 10^{-2}\right.$ radians).

\subsection{Horizontal Spectra}

We look for the horizontal spectrum of the atmospheric fluctuations by studying those parts of the lightcurves where the stellar image has an almost horizontal trajectory. We choose for that the Wise lightcurve because it has a good signal-to-noise ratio, it is relatively clear of aerosol, as noted before, and it remains far from the central flash region, where more complicated horizontal focusings occur. Figure 25 shows $\Phi_{\mathrm{sm}} / \Phi$ as a function of the distance $x$ along the limb for the entire occultation interval. Note that the stellar flux is detected well above the noise during all that time (see Fig. 4).

To obtain information on the horizontal structure of the atmosphere, we have to choose a region where the slope of the stellar trajectory is very shallow and, more precisely, smaller than the inverse of the aspect ratio of the stratospheric features. We have derived in the previous subsection typical aspect ratios of up to $\sim 200$, with a peak at $\sim 30 \pm 15$. We have thus restricted the calculation of the power spectra of $\left[\Phi_{\mathrm{sm}} / \Phi(x)\right]$ in regions of slopes smaller $1 / 200$ (corresponding to the horizontal solid line in Fig. 25). We have also performed tests with region where the slope is smaller than $1 / 50$ and $1 / 100$, noting little difference in the results.

As discussed in Appendix A, the resulting power spectrum must be corrected for projection effects. In particular, if this horizontal spectrum is a power law $\propto k_{\mathrm{h}}^{\mathrm{q}}$, where $k_{\mathrm{h}}$ is the modulus of the horizontal wavevector, then the apparent retrieved spectrum should be a power law with index $q+2$. Thus, when 


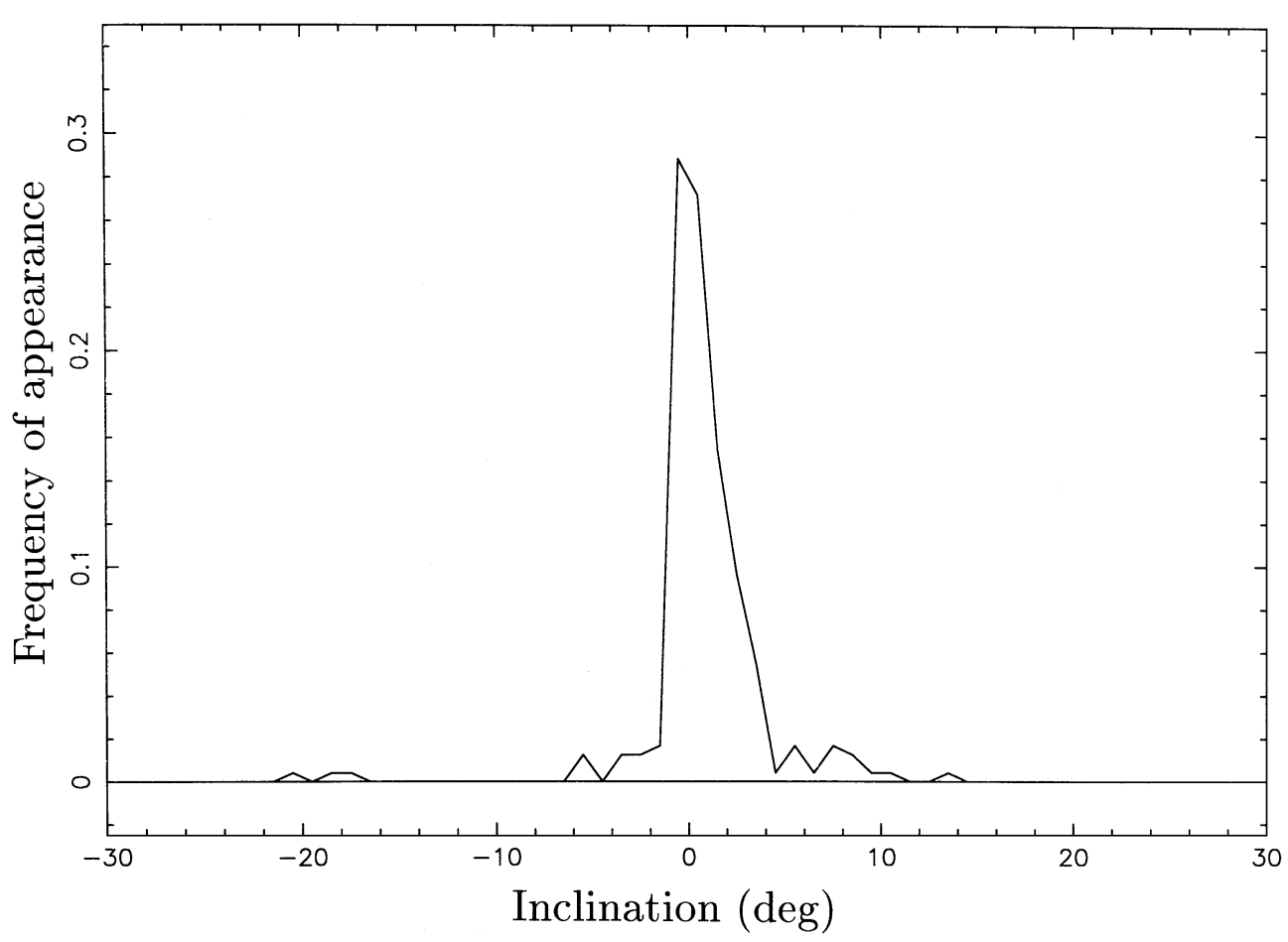

FIG. 24. Histogram of the inclinations of the atmospheric features with respect to the local horizontal, derived from the Ein Harod and Wise lightcurves (see the text).

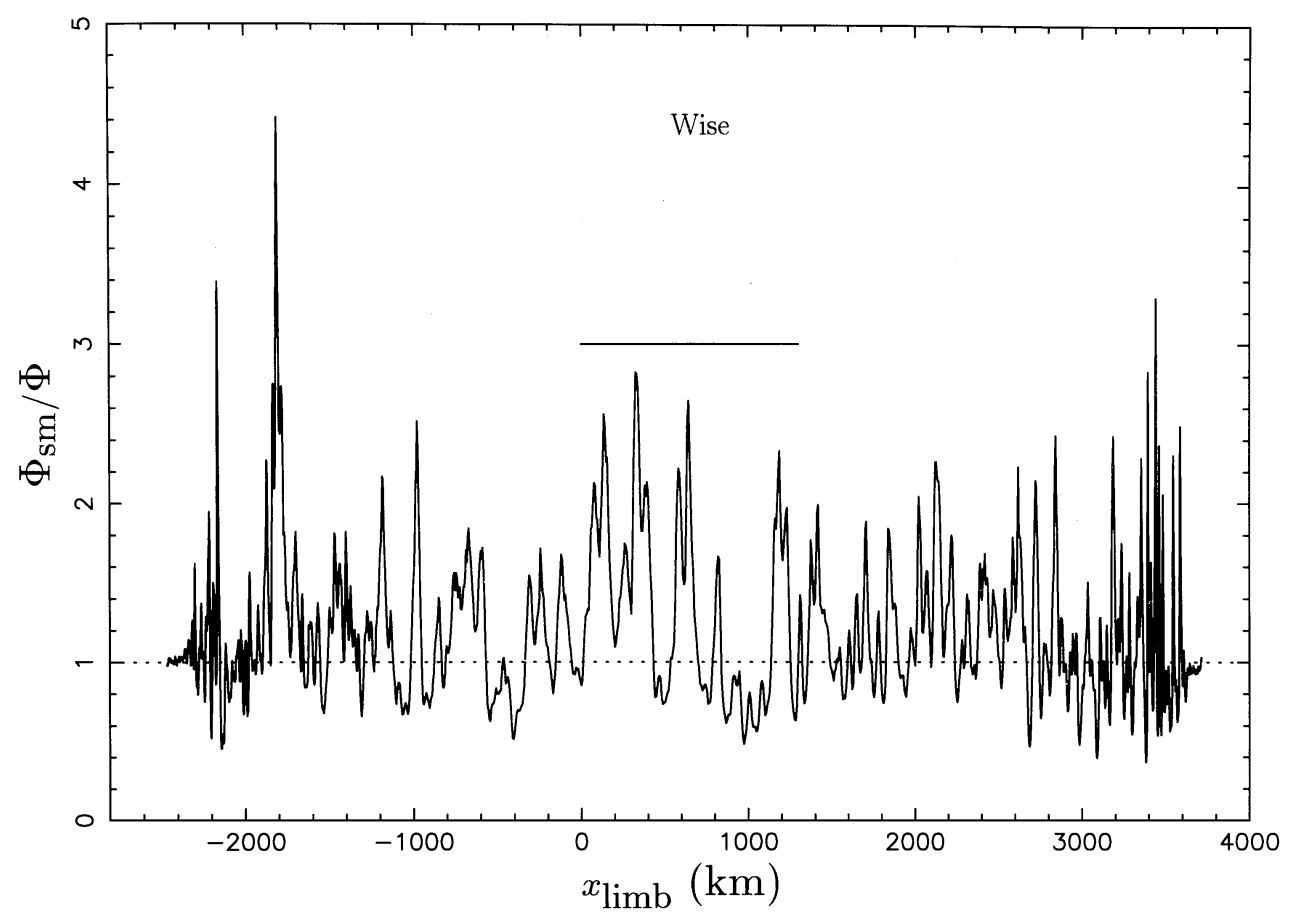

FIG. 25. Ratio $\Phi_{\mathrm{sm}} / \Phi$ of the smooth flux $\Phi_{\mathrm{sm}}$ expected from a Titan atmospheric model to the actual flux $\Phi$ as observed at the Wise station, as a function of the horizontal distance $x$ along the limb (see Appendix A for notation). The horizontal solid line denotes the interval where the slope of the stellar track is less than $1 / 200$ with respect to the local horizontal direction. 


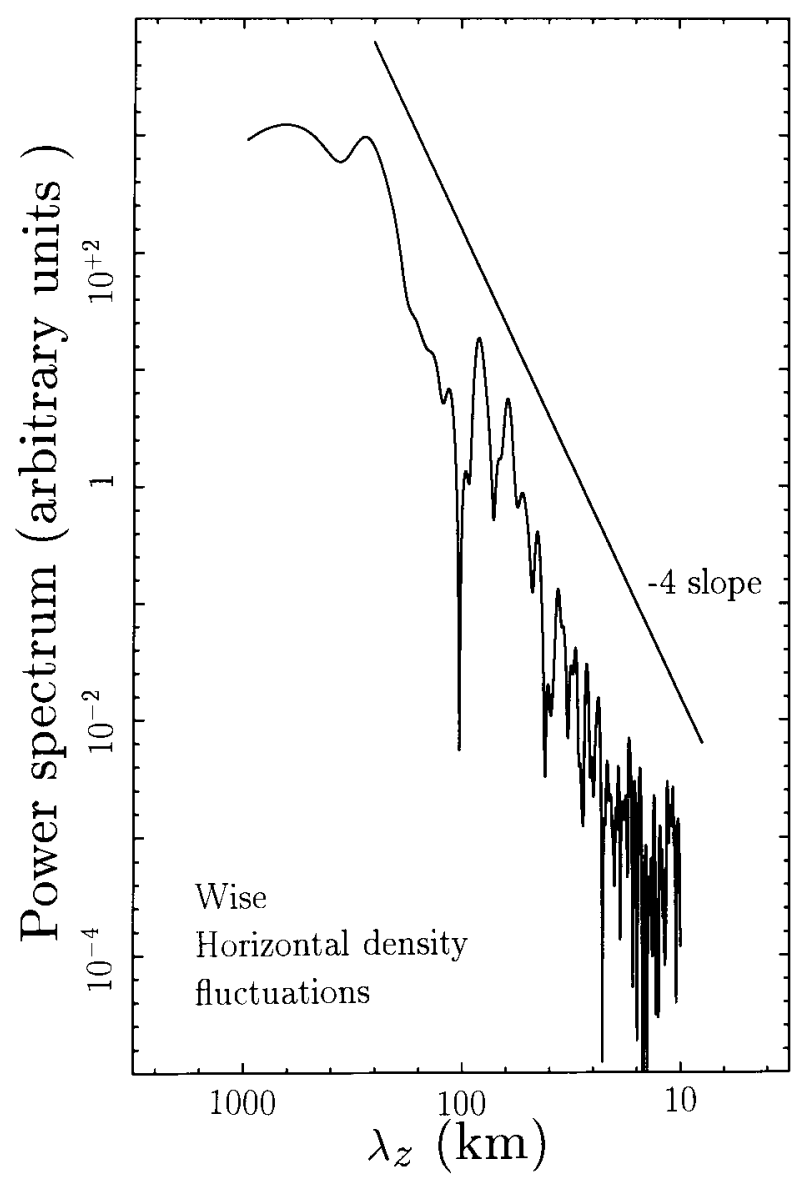

FIG. 26. Power spectrum of the ratio $\left[\Phi_{\mathrm{sm}} / \Phi\right](x)$ of Fig. 25, calculated in the interval corresponding to the horizontal solid line. This power spectrum has been divided by the square of the frequency to take into account projection effects, as explained in the text.

plotting the spectrum of Fig. 26, we have divided the power spectrum of $\left[\Phi_{\mathrm{sm}} / \Phi\right](x)$ by the square of the frequency, to take into account this correcting term of 2 .

The resulting slope observed in this figure is close to -4 . One has to remember that this result is derived from various assumptions described in Appendix A, namely, the separability of the vertical and horizontal spectra, the power law behavior of the horizontal spectrum, and its horizontal isotropy. Note, on the other hand, that smoothing by the finite stellar size should not be a problem here, since the structures that we are looking at have extensions much larger than the stellar diameter (see Fig. 25). Also, horizontal ray crossing should not be a problem either because we are far from the central flash region.

\section{DISCUSSION}

A better knowledge of the dynamical state of Titan's upper stratosphere can bring important constraints on the zonal wind generation and the energy budget at those levels. Also, these results can give some indications on the perturbations that will be suffered by the Huygens probe during its descent in the satellite atmosphere. This paper is mainly observational, and deals with the various biases that can affect the retrieved temperature and density profiles. Consequently, it is not our aim here to discuss all the theoretical and practical implications of our results. Instead, we summarize them, pointing out their domain of validity and their limitations. We note also that this study may be useful when comparing Titan's atmosphere with other upper atmospheres that show an activity, in particular, due to propagation of waves; see the abundant literature on gravity waves in the Earth middle atmosphere and evidence for wave activity in Venus (Hinson and Jenkins 1995), Jupiter (French and Gierasch 1974), Neptune (Hinson and Magalhães 1993, Roques, in preparation).

Global structure of the stratosphere. A dozen lightcurves obtained during the $28 \mathrm{Sgr}$ occultation have been used to study in detail the structure of Titan's atmosphere between altitude levels of $\sim 290$ and $500 \mathrm{~km}$ (corresponding to pressures from $\sim 110$ to $1.4 \mu$ bar, respectively). These lightcurves probe both the vertical and horizontal structures of the stratosphere. From a global point of view, we have seen that the retrieved density profiles, $n(z)$, agree well with the existing models (Lellouch et al. 1990, Yelle 1991), with a density scale height of $H=50.5 \pm 1.4 \mathrm{~km}$ between 300 and $500 \mathrm{~km}$ (Fig. 6), and with no significant difference between immersion and emersion. However, the observations cannot discriminate between the two models, since they are too close to each other in the region under consideration.

Density and temperature fluctuations. Once the smooth, basically exponential, variation of the density is taken away from the profiles, density (and corresponding temperature) fluctuations are detected well above the noise (Fig. 8). In particular, two conspicuous inversion layers, labeled $\mathrm{A}$ and $\mathrm{B}$, are visible in almost all the profiles, at altitudes of 425 and $450-455 \mathrm{~km}$, respectively ( $p \sim 7 \mu$ bar and $p \sim 4 \mu$ bar, respectively). These two layers correspond to sudden inversions of temperature, with increases of up to $\Delta T \sim 10 \mathrm{~K}$ in less than $\Delta z=10 \mathrm{~km}$ (Fig. 7), with local positive gradients $\partial T / \partial z$ of up to $2-3 \mathrm{~K} \mathrm{~km}^{-1}$.

As shown in Fig. 20, layer A and, to a lesser extent, layer B are found at very different latitudes on Titan (from $\sim 45^{\circ}$ south to $20^{\circ}$ north). In terms of density fluctuations, these features represent peak-to-peak relative variations of almost $10 \%$ over the altitude range of $10 \mathrm{~km}$ quoted in the previous paragraph (see Fig. 8).

The very nature of these strong inversion layers remains to be explained. Dips A and B observed in the lightcurves (Fig. 3) are probably not caused by absorptions by detached aerosol layers, because of the absence of chromatic effects between the blue and red channels of the various experiments. As discussed below, ray crossing cannot account for this structure either. Also, the fact that the region joining these two inversion layers just reaches the adiabatic lapse rate (Fig. 9) points toward a dynamical origin for these features.

Other examples of such inversion layers are indeed encountered in planetary upper atmospheres, but their origin is still a 
topic of debate. For instance, lidar observations of the Earth mesophere reveal a strong inversion layer, with similar amplitude, and a cutoff at the adiabatic lapse rate around the altitude 70-75 km, i.e., pressures of about 45-20 $\mu$ bar (Hauchecorne et al. 1987). These authors interpret the maintenance of the inversion layer by the continuous breaking of gravity waves entering the inversion region. Inversion layers are also observed in middle atmosphere of Mars in the altitude range 40-60 km (pressures $\sim 130-13 \mu$ bar), with a similar interpretation in terms of gravity wave activity (Théodore et al. 1993).

Smaller variations are also clearly visible in Fig. 8, and all of them can have an effect on the Huygens probe acceleration during its descent in the atmosphere of the satellite. Conversely, the Huygens Atmospheric Structure Instrument (HASI) experiment (which yields the pressure and temperature versus altitude), combined with the accelerometer data, could provide interesting insights concerning these structures, and extend this study to higher and lower zones of the atmosphere (Fulchignoni 1992, Strobel and Sicardy 1997).

Actually, the amplitudes of the fluctuations observed in our data are consistent with the presence of gravity waves reported by other authors lower down in the atmosphere (Hinson and Tyler 1983, Friedson 1994). According to Friedson, the energy flux of a gravity wave in the WKB approximation is $\propto \rho^{\prime 2} /\left(N^{3} \rho_{0}\right)$, where $\rho^{\prime}$ is the perturbation mass density of the wave, $\rho_{0}$ is the background unperturbed density, and $N$ is the Brunt-Vaisala frequency. Thus, if dissipation is ignored, the relative amplitude of the wave varies like $\rho^{\prime} / \rho_{0} \propto N^{3 / 2} / \rho_{0}^{1 / 2}$. (Note that this formula also ignores the effect of wind shear, which can be very significant.) Around $44 \mathrm{~km}$, the scintillation of the radio signal sent by the Voyager spacecraft indicates $\rho^{\prime} / \rho_{0} \sim 7 \times 10^{-4}$ (Hinson and Tyler 1983), while $\rho_{0} \sim 5.2 \times 10^{-4} \mathrm{~g} \mathrm{~cm}^{-3}$ and $N \sim 0.005 \mathrm{~s}^{-1}$ at that level. If the gravity waves propagate upward with constant energy up to inversion layer $\mathrm{A}(425 \mathrm{~km})$, where $\rho_{0} \sim 1.3 \times$ $10^{-8} \mathrm{~g} \mathrm{~cm}^{-3}$ and $N \sim 0.0024 \mathrm{~s}^{-1}$, then the equations above predict typical relative amplitudes for the waves of $\rho^{\prime} / \rho_{0} \sim 5 \times$ $10^{-2}$. These are actually the amplitudes observed in Fig. 8 .

Thus, the fluctuations observed in our data are consistent with the upward propagation of gravity waves from deeper layers. In particular, in the region under study, the amplitude of some the waves can reach the critical value which causes convective instability and breaking.

Cutoff of the temperature gradients. The temperature gradients $\partial T / \partial z$ clearly show a cutoff near the adiabatic lapse rate $\Gamma \sim-1 \mathrm{~K} \mathrm{~km}^{-1}$, while spikes can reach positive values of up to $3 \mathrm{~K} \mathrm{~km}^{-1}$ (Figs. 9, 10). Altogether, these results suggest that ongoing breaking of gravity waves, due to convective instability, is taking place in this region of the atmosphere, as noted above.

The asymmetry of the $\partial T / \partial z$ profiles is more difficult to explain since it depends on the detail of how the waves break. We have considered an alternative possibility to account for this asymmetry, namely, ray crossing (Figs. 15, 16). Note that we have no independent way of distinguishing in our observations between ray crossing and asymmetries caused by breaking waves. Furthermore, Eq. (7) indicates that ray crossing and con- vective instability occur at about the same amplitude for structures with the vertical wavelengths detected here (from a few kilometers to some $50 \mathrm{~km}$ ). This obviously complicates the interpretation of diagrams like Figs. 9 and 10.

Nevertheless, there is no reason why ray crossing should simulate a cutoff so close to $\Gamma$, as shown in Fig. 10. Thus, even though ray crossing could be responsible for some of the morphology observed in the $\partial T / \partial z$ profiles, the actual negative gradients must be close to the adiabatic limit in any case. The strong cutoff $\partial T / \partial z$ by $\Gamma$ has indeed been observed in other atmospheres; see the lidar observations of the Earth's mesosphere (Hauchecorne et al. 1987), the radio occultation sounding of Venus' during the Magellan flyby (Hinson and Jenkins 1995), and ground-based stellar occultations by Neptune (Roques, in preparation).

Vertical power spectra. Another test as to the nature of the fluctuations can be performed through the power spectra of the vertical profiles of $n(z)$ or $T(z)$. Figures 11 and 17 show a robust power law behavior, with an exponent close to -3 for wavelengths larger than $\sim 10 \mathrm{~km}$ and close to -4 for higher frequencies. There is a concern, however, about the physical reality of this slope, because ray crossing may occur in some regions of the profiles, due especially to high-frequency features. As discussed in Section 7, ray crossing steepens the original spectrum. Consequently, we cannot distinguish at our level a -2 spectrum modified by ray crossing as in Fig. 15B (right), a real -3 spectrum as in Fig. 16A (right), or a mixture of the two, when compared with real data (right panel of Fig. 17). However, our simulations clearly indicate that the original spectrum cannot have a flat shape nor a -4 slope, so that the real vertical spectrum must have a slope between -2 and -3 .

The -3 slope is reminiscent of the so-called "universal -3 spectrum" found in the Earth oceans (Bell 1975, Garrett and Munk 1975) and middle atmosphere (Smith et al. 1987), as well as in Neptune's stratosphere (Roques, in preparation). Its origin seems to be linked to the presence of gravity waves propagating upward in a stratified atmosphere, but the detailed mechanisms responsible for such a spectrum are not yet clearly established. Explanations range from breaking of individual wave packets to Doppler-shifted couplings between the horizontal winds forced by the waves or radiation (see the reviews by Gardner 1994 and Zhu 1994). In our case, the fact that the adiabatic lapse rate is almost reached in some places and the asymmetry in the gradient distribution suggest that breaking due to convective instabilities is actually taking place and must dominate the resulting spectra.

Horizontal layering. The comparison of various profiles probing regions separated by some tens to hundreds of kilometers allowed us to estimate the aspect ratio (ratio of horizontal-tovertical typical scales) of the observed structures. While layers A and $\mathrm{B}$ exhibit a strong correlation from one profile to the other, as discussed before, smaller structures are lost progressively with increasing horizontal distance (see Fig. 21). The comparison of the Israeli lightcurves (Ein Harod and Wise) allowed us to follow this gradual loss of correlation between the structures as they are probed more and more horizontally. 
This gradual loss indicates typical aspect ratios between 15 and 45, and even more for some features, as seen in Fig. 23. Also, cross-correlation of profiles for stations from Manley to Pic du Midi indicates aspect ratios of about 140 at altitudes of 360 to $415 \mathrm{~km}$ (see Fig. 21). Altogether, this reveals a highly stratified atmosphere. These numbers can be compared with the typical values of $\rho \sim 25-100$ found by Narayan and Hubbard (1988) for Neptune's stratosphere, from the analysis of stellar scintillation near ingress, egress, and central flash. Note that the observed layers in Titan's stratosphere are essentially horizontal, with a small dispersion of about $\pm 2.5 \times 10^{-2}$ radian $\left(\sim \pm 1.5^{\circ}\right)$ with respect to the local horizontal plane (Fig. 24).

Horizontal power spectrum. In the most horizontal parts of its track, the stellar image is modulated by the horizontal structure of the atmosphere only (see Appendix A). However, we then observe a two-dimenional field of horizontal fluctuations projected along a one-dimenional line (the limb of Titan) in the plane of the sky. The observed "projected" horizontal spectrum has a slope of $\sim-2$, and some assumptions are then necessary to deduce the actual horizontal power spectrum. Namely, if we assume the separability of the vertical and horizontal spectra, the isotropy of the horizontal field of fluctuations, and a power law variation for the horizontal spectrum, we can infer a slope close to -4 for the actual horizontal power spectrum (see Fig. 26).

The comparisons of the vertical and horizontal power spectra obtained here may be important steps in understanding the very nature of these fluctuations, their mechanisms of interaction, and their temporal spectrum, as discussed in detail by Gardner (1994) and Daubner and Zeitlin (1996).

\section{APPENDIX A: EFFECTS OF FLUCTUATIONS}

Let us consider a planetary atmosphere whose density decreases exponentially with altitude, with a constant scale height $H$. We add to this smooth atmospheric profile three-dimensional (3D) density (and also refractivity) fluctuations, and we look for the flux eventually received by the observer. We treat the problem in the frame of geometric optics, a valid assumption as long as we consider structures larger than the Fresnel scale $F=\sqrt{\lambda D}$, where $\lambda$ is the wavelength of observation and $D$ the distance of the planet to the observer. In the visible and in the case of Titan $\left(D=1.35 \times 10^{9} \mathrm{~km}\right), F$ is of the order of $1 \mathrm{~km}$. Because the projected stellar diameter at Titan $(\sim 18 \mathrm{~km})$ is larger than $F$, the geometric optics approach is sufficient for our purpose.

The basic geometric of the problem is shown in Fig. A1. Note that the angle of deviation, $\omega$, is negative with our conventions and very small, e.g., always less than $\sim 2 \times 10^{-6}$ radian in the case of Titan. Consequently, $A$ can be considered as the distance of the closest approach of the ray to the planet center, $O$, to an accuracy better than $1 \mathrm{~m}$. Let $r$ be that closest distance, and let $r^{\prime}$ be the distance of the observer $B$ to the center of the shadow, $O^{\prime}$. Also, due to the very small value of the deviation angle $\omega$, the trajectory of the ray inside the atmosphere can be considered as a straight line going through $A$ and parallel to $O y$. This is the large value of $D$, despite the smallness of $\omega$, which is eventually responsible for the drop of signal at the observer.

Because the fluctuations of density may have a locally horizontal component, the ray also suffers deviations along the third dimension $O x$, perpendicular to the plane of Fig. A1, i.e., along the limb of the planet as seen from the observer. One can, however, show that these horizontal deviations can be neglected with respect to the vertical ones as long as the observer is far from the center of the shadow, i.e., outside the region of the so-called "central flash" (see, e.g., Hubbard et al. 1988).

In these conditions, the angle of deviation is given by

$$
\omega(x, r)=\int_{-\infty}^{+\infty} \frac{\partial v}{\partial r} \cdot d s \sim \int_{-\infty}^{+\infty} \frac{\partial v(x, y, r)}{\partial r} \cdot d y,
$$

where $v(x, y, r)$ is the refractivity of the atmosphere at a given point along the

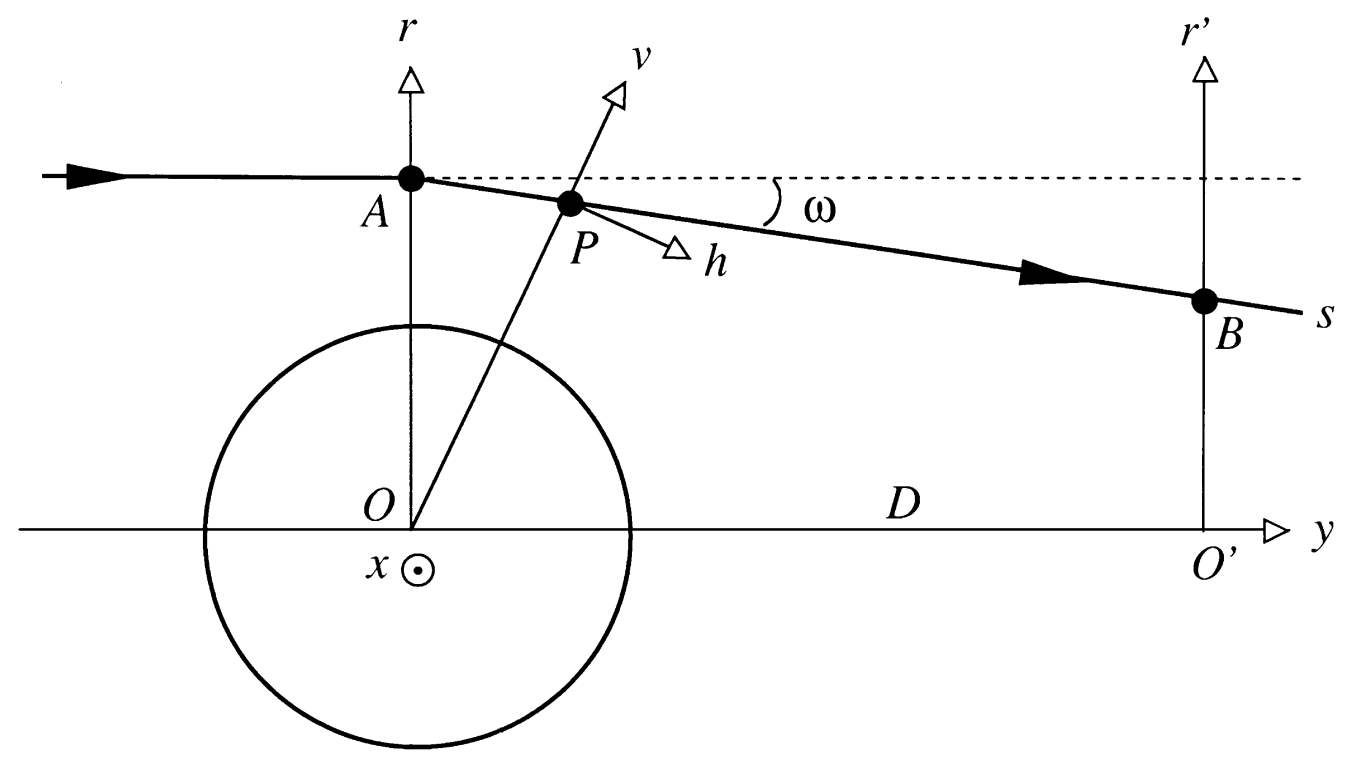

FIG. A1. Basic geometry of a ray deviation during a stellar occultation by a planetary atmosphere. The center of the planet is at $O, O y$ is directed along the direction of the incoming stellar rays. The vertical axis $O r$ intercepts that incoming ray at $A$. The axis $O x$ (not visible here) is perpendicular to $O y$ and $O r$. Thus, $O x$ is directed along the limb of the planet, as seen from the observer $B$, and $O x r$ defines the plane of the sky. After being slightly refracted by an angle $\omega$, the ray moves along the general direction $A s$, over a distance $D$, and eventually hits the observer $B$ who is moving along the axis $O r^{\prime}$. The axis $O v$ defines the direction of the local vertical at a given point $P$ in the planetary atmosphere, and $P h$ defines the direction of the local horizontal at $P$, in the plane of the figure. 
ray. As noted above, $v$ can be decomposed into a smooth component $v_{0}$, varying exponentially with altitude, and a fluctuation $\delta v$ :

$$
v=v_{0}+\delta v
$$

Note that even though the background atmosphere is supposed to have an exponential density profile, this does not mean that it is isothermal. The ray hits the observer at $r^{\prime}=r+D \omega$, and from conservation of energy, the observed flux is

$$
\Phi=f \frac{d r}{d r^{\prime}}=\frac{f}{1+D(\partial \omega / \partial r)},
$$

where $f$ is the focusing factor due to the curvature of the limb. Let $\Phi_{0}$ be the output flux caused by the profile $\nu_{0}$, i.e., a Baum and Code lightcurve. Finally, let $\Phi_{\mathrm{sm}}=f \Phi_{0}$ be the smooth lightcurve that combines the Baum and Code function and the focusing due to limb curvature. Then,

$$
\frac{\Phi_{\mathrm{sm}}}{\Phi}=1+D \Phi_{0} \frac{\partial \delta \omega}{\partial r} \sim 1+D \Phi_{0} \int_{-\infty}^{+\infty} \frac{\partial^{2} \delta v}{\partial r^{2}} \cdot d y .
$$

Now, the fluctuation $\delta v$ is expressed more easily as a function of the local coordinates $(x, h, v)$, where $x$ is the distance along the limb, as mentioned before, $v$ is the distance to the planet center along the local vertical direction, and $h$ is the circular distance along the local horizontal direction in the plane of the figure. We consider strongly stratified fluctuations $\delta v(x, h, v)$, i.e., $\partial v / \partial v \gg$ $\partial v / \partial x, \partial v / \partial h$.

Effect of a monochromatic $3 D$ wave. More specifically, we take the following form for $\delta v$ :

$$
\delta v(x, h, v)=|\delta v(v)| \cdot \sin (k x+l h+m v),
$$

where the amplitude $|\delta v(v)|$ varies slowly with altitude like $|\delta v(v)| \propto \exp (-v /$ $H^{\prime}$ ). We assume that $H$ and $H^{\prime}$ are of the same order of magnitude, but not necessarily equal. Thus the fluctuation $\delta v$ is a $3 \mathrm{D}$ monochromatic wave with wavevector $(k, l, m)$. The strong stratification assumption means that $m \gg k, l$, and we also assume $m \gg 1 / H^{\prime}$. If $H=H^{\prime}$, the amplitude of the fluctuation relative to the background value $\nu_{0}$ is constant with height. If the fluctuation is a propagating gravity wave that conserves the energy flux, then $H^{\prime}=2 H$, and the relative amplitude of the wave grows as $\exp \left(v / H^{\prime}\right)$ with height.

Most of the deviation of the ray comes from a horizontal segment of length $L \sim \sqrt{2 \pi r H} \ll r$ around $A$, where the atmosphere is the densest. Also, the vertical gradient of $v$ is assumed to be much larger than horizontal gradients. From these two properties, one can deduce that

$$
\frac{\Phi_{\mathrm{sm}}}{\Phi} \sim 1+D \Phi_{0} \int_{-\infty}^{+\infty} \frac{\partial^{2} \delta v}{\partial v^{2}} \cdot d y .
$$

Furthermore, $h \sim y$ and $v \sim r+y^{2} / 2 r$ in the region of interest. From $\partial^{2} v / \partial v^{2} \sim$ $-m^{2} v$, one finally gets

$$
\begin{aligned}
\frac{\Phi_{\mathrm{sm}}}{\Phi}(x, r) \sim & 1-D \Phi_{0} m^{2}|\delta v(r)| \int_{-\infty}^{+\infty} \exp \left(-y^{2} / 2 r H^{\prime}\right) \\
& \cdot \sin \left(l y+m y^{2} / 2 r+k x+m r\right) \cdot d y \\
= & 1-D \Phi_{0} m^{2} \sqrt{2 r H^{\prime}}|\delta v| \int_{-\infty}^{+\infty} \exp \left(-u^{2}\right) \\
& \cdot \sin \left[m H^{\prime}\left(u+\sqrt{\frac{r l^{2}}{2 H^{\prime} m^{2}}}\right)^{2}+k x+m r-\frac{r l^{2}}{2 m}\right] \cdot d u .
\end{aligned}
$$

We now use the identity

$$
\begin{aligned}
& \int_{-\infty}^{+\infty} \exp \left(-u^{2}\right) \cdot \sin \left[\tan \alpha \cdot(u+a)^{2}+\phi\right] \cdot d u \\
& \quad=\sqrt{\pi \cos \alpha} \cdot \exp \left(-a^{2} \sin ^{2} \alpha\right) \cdot \sin \left(a^{2} \sin \alpha \cos \alpha+\alpha / 2+\phi\right) .
\end{aligned}
$$

In our case, $\tan \alpha=m H^{\prime} \gg 1$, so that $\cos \alpha \sim 1 / m H^{\prime}$ and $\alpha \sim \pi / 2$. Consequently,

$$
\begin{aligned}
& \frac{\Phi_{\mathrm{sm}}}{\Phi} \sim 1-D \Phi_{0} m^{3 / 2} \sqrt{2 \pi r}|\delta v| \cdot \exp \left[-\frac{r}{2 H^{\prime}}\left(\frac{l}{m}\right)^{2}\right] \\
& \cdot \sin \left(k x+m r-\frac{r l^{2}}{2 m}+\frac{\pi}{4}\right) .
\end{aligned}
$$

Let

$$
\epsilon(r)=\frac{|\delta v|}{v_{0}}
$$

be the relative amplitude of the fluctuation. The Baum and Code flux $\Phi_{0}$ is related to $v_{0}$ through

$$
v_{0}=\frac{1-\Phi_{0}}{\Phi_{0}} \sqrt{\frac{H^{3}}{2 \pi D^{2} r}},
$$

so that

$$
\begin{gathered}
\frac{\Phi_{\mathrm{sm}}}{\Phi}(x, r) \sim 1-\left(1-\Phi_{0}\right)(m H)^{3 / 2} \cdot \exp \left[-\frac{r}{2 H^{\prime}}\left(\frac{l}{m}\right)^{2}\right] \\
\cdot \epsilon(r) \cdot \sin \left(k x+m r-\frac{r l^{2}}{2 m}+\frac{\pi}{4}\right) .
\end{gathered}
$$

Highly stratified atmosphere. Note that the exponential factor damps the amplitude of the fluctuations. The argument of this exponential term is $\left(r / 2 H^{\prime}\right)$ $(l / m)^{2}=\left(L / H^{\prime}\right)^{2}\left(\lambda_{\mathrm{v}} / \lambda_{\mathrm{h}}\right)^{2} / 4 \pi$, where $L \sim \sqrt{2 \pi r H^{\prime}}$ is the horizontal length in the atmosphere where most of the deviation takes place, and $\lambda_{\mathrm{v}}$ and $\lambda_{\mathrm{h}}$ are the vertical and horizontal wavelengths of the wave, respectively. Taking $H^{\prime}=2 H \sim 100 \mathrm{~km}$ and $L \sim 1400 \mathrm{~km}$ for Titan, we have $\left(r / 2 H^{\prime}\right)(l / m)^{2} \sim$ $15\left(\lambda_{\mathrm{h}} / \lambda_{\mathrm{V}}\right)^{-2}$. Since the aspect ratio of the observed waves, $\lambda_{\mathrm{h}} / \lambda_{\mathrm{V}}$, is larger than $\sim 30$, the factor $\left(r / 2 H^{\prime}\right)(l / m)^{2}$ remains well below unity. Thus, the damping factor due to the horizontal structure of the wave is actually negligible since the exponential above is very close to unity.

This shows that even horizontal wavelengths (along the line of sight) small with respect to $L$ are inefficient in damping the ouput flux fluctuations, provided that the aspect ratio $\lambda_{\mathrm{h}} / \lambda_{\mathrm{v}}$ remains large. We thus eventually obtain

$$
\begin{aligned}
\frac{\Phi_{\mathrm{sm}}}{\Phi}(x, r) \sim & 1-\left(1-\Phi_{0}\right)(m H)^{3 / 2} \\
& \cdot \epsilon(r) \cdot \sin \left(k x+m r-\frac{r l^{2}}{2 m}+\frac{\pi}{4}\right) .
\end{aligned}
$$

In these conditions, the effect of the horizontal wavelength $\lambda_{\mathrm{h}}=2 \pi / l$ is a mere dephasing, $-r l^{2} / 2 m$, with no effect on the amplitude of the fluctuation.

Ray crossing. When ray crossing occurs, caustics are encountered and the flux $\Phi$ diverges. The expression above thus provides the following criterion for ray crossing to appear

$$
\left(1-\Phi_{0}\right)(m H)^{3 / 2} \epsilon \geq 1,
$$


a result previously derived by French and Lovelace (1983) in the particular case of a perfectly layered atmosphere $(k=l=0)$.

Spectrum of fluctuations. More generally, if we have a spectrum of fluctuations of the form:

$$
\begin{aligned}
\frac{\delta v}{v_{0}}(x, h, v)= & \frac{1}{(2 \pi)^{3}} \iiint_{m, k, l} \tilde{\epsilon}(m, k, l) \\
& \cdot \exp [-i(k x+l h+m v)] \cdot d m d k d l,
\end{aligned}
$$

then the ratio $\Phi_{\mathrm{sm}} / \Phi$ is

$$
\begin{gathered}
\frac{\Phi_{\mathrm{sm}}}{\Phi}(x, z)=1-\frac{\left(1-\Phi_{0}\right)}{(2 \pi)^{3}} \iiint_{m, k, l}(m H)^{3 / 2} \tilde{\epsilon}(m, k, l) \\
\cdot \exp [-i(k x+m z+\varphi)] \cdot d m d k d l
\end{gathered}
$$

where $\varphi$ absorbs the various constant phases of the problem. Equation (A4) shows that the power spectrum of $\Phi_{\mathrm{sm}} / \Phi$ is directly proportional to $m^{3} \mid \tilde{\epsilon}(m$, $k, l)\left.\right|^{2}$. In these regions of the stellar trajectory where $m z$ is varying much more rapidly than $k x$ (ingress and egress), then the power spectrum of $\Phi_{\mathrm{sm}} / \Phi$ is $\propto m^{3}|\tilde{\epsilon}(m)|^{2}$. We have already derived the vertical power spectra of density fluctuations (see Fig. 11) and found a power law with a $\sim-3$ slope. We thus expect a flat vertical power spectrum for $\left[\Phi_{\mathrm{sm}} / \Phi\right](z)$, which is actually the case on examination of various examples near ingress or egress.

After this check, we turn to the horizontal power spectrum of $\left[\Phi_{\mathrm{sm}} / \Phi\right](x)$. There are several difficulties in interpreting this spectrum, however. First, if we want to derive simple conclusions as to the dependence of $|\tilde{\epsilon}(m, k, l)|^{2}$ on $k$ and $l$, we have to assume that $|\tilde{\epsilon}|$ is separable with respect to the vertical and horizontal directions, i.e., $|\tilde{\epsilon}(m, k, l)|=\left|\tilde{\epsilon}_{\mathrm{v}}(m)\right| \times\left|\tilde{\epsilon}_{\mathrm{v}}(k, l)\right|$. This assumption seems a reasonable one if the fluctuations are due to a field of saturated gravity waves (see the discusion by Daubner and Zeitlin 1996). Another assumption is that the field of fluctuation is isotropic in the horizontal direction, i.e., that $\left|\tilde{\epsilon}_{\mathrm{v}}(k, l)\right|$ depends only on the modulus of the horizontal wavenumber, $k_{\mathrm{h}}=\sqrt{k^{2}+l^{2}}$. To simplify the problem further, we assume that this dependence is a power law of exponent $q$ for $\left|\tilde{\epsilon}_{\mathrm{v}}\left(k_{\mathrm{h}}\right)\right|^{2}$, i.e., that $\left|\tilde{\epsilon}_{\mathrm{v}}\left(k_{\mathrm{h}}\right)\right| \propto\left(k^{2}+l^{2}\right)^{q / 4}$.

A final difficulty then appears, namely, that we observe only a "projection" of the density fluctuations onto the plane of the sky. Equation (A2) shows that the dependence of $\Phi_{\mathrm{sm}} / \Phi$ on the third dimension $h$ (along the line of sight) has disappeared, except for a mere shift in phase of $-r l^{2} / 2 m$. When integrating the Eq. (A4) with respect to the variable $m$, and taking into account the assumptions described above, one gets

$$
\frac{\Phi_{\mathrm{sm}}}{\Phi}(x)=1-C \iint_{k, l}\left(k^{2}+l^{2}\right)^{\mathrm{q} / 4} \cdot \exp (-i k x) \cdot d k d l
$$

where the complex number $C$ absorbs the various constants and phases of the problem. The integral above can be calculated in polar coordinates, i.e., writing $l=k_{\mathrm{h}} \sin \theta=k \tan \theta$; thus,

$$
\begin{aligned}
\frac{\Phi_{\mathrm{sm}}}{\Phi}(x)= & 1-C \int_{k} \int_{\theta=0}^{\theta=2 \pi} k^{(q+2) / 2} \\
& \cdot[\cos \theta]^{-(q+4) / 2} \cdot \exp (-i k x) d k d \theta
\end{aligned}
$$

Consequently, the projection effects transform an original power spectrum in $k_{\mathrm{h}}$ of slope $q$ into an "apparent" power spectrum in $k$ of slope $q+2$. This effect must then be taken into account when deriving the power spectrum of the horizontal fluctuations (see the main text).

\section{APPENDIX B: VERTICAL POWER SPECTRA}

We want to estimate here the power spectrum of the function $f(z)$. The quantity $f$ can be the temperature or the density fluctuation, and $z$ can be the altitude, but also the distance $x$ along the limb of Titan. Let $\lambda_{z}$ be the wavelength, and $m=2 \pi / \lambda_{\mathrm{z}}$ the wavenumber. The power spectrum of $f$ is $P(m)=|\tilde{f}(m)|^{2}$, where $\tilde{f}(m)$ is the Fourier transform of $f$, defined as

$$
\tilde{f}(m)=\int_{-\infty}^{+\infty} f(z) e^{i m z} d z
$$

What we have in reality is a profile in a finite interval $\left(z_{\min }, z_{\max }\right)$ at $N$ discrete points $z_{\mathrm{k}}$ distributed (not regularly) between $z_{\min }$ and $z_{\max }$. We use the code described by Deeming (1975) to estimate $\tilde{f}(m)$. This code provides the following outputs:

$$
\begin{aligned}
& \tilde{f}_{N}(m)=\frac{1}{N} \sum_{k=1}^{N} f\left(z_{k}\right) e^{i m z_{k}} \\
& \gamma_{N}(m)=\frac{1}{N} \sum_{k=1}^{N} e^{i m z_{k}},
\end{aligned}
$$

where $\gamma_{N}(m)$ is called the spectral window. The "numerical" Fourier transform $\tilde{f}_{N}(m)$ is related to the true Fourier transform $\tilde{f}(m)$ by $\tilde{f}_{N}=\gamma_{N} * \tilde{f}$, where $*$ denotes the convolution. Although we have access to $\tilde{f}_{N}(m)$ only, we loosely refer to $P_{N}(m)=\left|\tilde{f}_{N}(m)\right|^{2}$ as the "power spectrum" of $f$, or even the "spectrum" for short.

The general shape of $P_{N}(m)$ must be interpreted with some care. Several problems can alter the original spectrum: (i) an irregular sampling of the profile (due in our case to the varying velocity of the stellar image in the atmosphere), which introduces a complex aliasing; (ii) the finite interval over which the spectrum is calculated, $\Delta z=z_{\max }-z_{\min }$, which causes a "windowing effect," cutting off low frequencies; (iii) the stellar diameter, which smoothes out high frequencies; and (iv) ray crossings, which distort the derived refractivity profiles and thus affect the estimated spectrum.

To counteract point (i), the profiles are first resampled by linear interpolation at a regular step of $0.1 \mathrm{~km}$, in the case of the vertical temperature profiles. This is well below the apparent stellar diameter, which can shrink down to $\sim 1.5 \mathrm{~km}$ at the bottom of the profiles, near $z=310 \mathrm{~km}$.

The windowing effect [point (ii)] can be minimized by multiplying the entire profile by a Hanning function. The latter replaces the original profile $f\left(z_{k}\right)$ by $f\left(z_{k}\right) \times\left\{1-\cos \left[2 \pi\left(z_{\max }-z_{\min }\right) / \Delta z\right]\right\}$, tapering the edge of the input profile and reducing spikes in the calculated spectrum. This is similar to apodization in optics to reduce the diffraction pattern. Low-frequency terms can also be artificially introduced in the profile during the inversion (through arbitrary initial conditions). They are removed by subtracting a low-degree polynomial from the profile. This ensures that the power spectrum goes to zero as the frequency goes to zero (see, e.g., Fig. 14). We have performed tests that show that windowing effects are satisfactorily removed using polynomials of degree 4 over the vertical interval $z=310-500 \mathrm{~km}$, where most of the profiles were considered. The price to pay for this is that we lose the spectral information for wavelengths larger than $\sim 50 \mathrm{~km}$ (i.e., one scale height) and smaller than $\sim 3 \mathrm{~km}$, (see, e.g., Fig. 13).

The apparent stellar diameter [point (iii)] will, on the other hand, remove high-frequency terms, as shown again in Fig. 13. Ray crossings can also steepen the spectrum [point (iv)]. This effect is illustrated in Figs. 14 to 16, and is discussed in the main text.

\section{ACKNOW LED GMENTS}

The authors thank J. L. Elliot and D. P. Hinson for numerous and constructive criticisms on the original manuscript. We also acknowledge fruitful and enlightening discussions with F. Dalaudier, R. G. French, A. J. Friedson, P. J. Gierasch, P. Goldreich, E. Lellouch, R. V. E. Lovelace, J. R. Lyons, D. F. Strobel, and V. Zeitlin. W. B. Hubbard acknowledges support from NASA Grant NAG5-4214. Observations at the Wise Observatory are supported in part by grants from the Israel Science Foundation. 
Note. We can make available on demand (at sicardy@mesiob.obspm.fr) the original lightcurves, as well as the density and temperature profiles reported here, either as input data in engineering models of the Huygens probe or for theoretical studies of Titan's stratosphere dynamics.

\section{REFERENCES}

Beisker, W., C. Bittner, H.-J. Bode, R. Buechner, H. Denzau, D. Dunham, and E. Riedel 1989. The occultation of 28 Sgr by Titan: First results. Occultation Newslett. 4, 324-326.

Bell, T. H. 1975. Topographically generated internal waves in the open oceans. J. Geophys. Res. 80, 320-327.

Bretagnon, P. 1982. Théorie du mouvement de l'ensemble des planètes: Solution VSOP82. Astron. Astrophys. 114, 278-288.

Daubner, S., and V. Zeitlin 1996. On the stationary energy spectra for unidirectionally propagating internal gravity waves. Phys. Lett. A 214, 33-39.

Deeming, T. J. 1975. Fourier analysis with unequally-spaced data. Astrophys. Space Sci. 36, 137-158.

Dubouloz, N., F. Raulin, E. Lellouch, and D. Gautier 1989. Titan's hypothesized ocean properties: The influence of surface temperature and atmospheric composition uncertainties. Icarus 82, 81-96.

Forrest, R. W., and I. K. M. Nicolson 1990. Photometric observations of the occultation of $28 \mathrm{Sgr}$ by Titan. Mon. Not. R. Astron. Soc. 243, 10-13.

French, R. G., and P. J. Gierasch 1974. Waves in the jovian upper atmosphere. J. Atmos. Sci. 31, 1707-1712.

French, R. G., and R. V. E. Lovelace 1983. Strong turbulence and atmospheric waves in stellar occultations. Icarus 56, 122-146.

French, R. G., P. D. Nicholson, M. L. Cooke, J. L. Elliot, K. Matthews, O. Perković, E. Tollesstrup, P. Harvey, N. J. Chanover, M. A. Clark, E. W. Dunham, W. Forrest, J. Harrington, J. Pipher, A. Brahic, I. Grenier, F. Roques, and M. B. Arndt 1993. Geometry of the Saturn system from the 3 July 1989 occultation of $28 \mathrm{Sgr}$ and Voyager observations. Icarus 103, 163-214.

Friedson, A. J. 1994. Gravity waves in Titan's atmosphere. Icarus 109, 40-57.

Fulchignoni, M. 1992. The atmosphere of Titan and the Huygens atmospheric structure instrument. Nuovo Cimento 15C, 1163-1176.

Gardner, C. S. 1994. Diffusive filtering theory of gravity wave spectra in the atmosphere. J. Geophys. Res. 99, (20)601-(20)622.

Garrett, C., and W. Munk 1975. Space-time scales of internal waves: A progress report. J. Geophys. Res. 80, 291-297.

Hauchecorne, A., M. L. Chanin, and R. Wilson 1987. Mesospheric temperature inversion and gravity wave breaking. Geophys. Res. Lett. 14, 933-936.

Hinson, D. P., and J. M. Jenkins 1995. Magellan radio occultation measurements of atmospheric waves on Venus. Icarus 114, 310-327.

Hinson, D. P., and J. A. Magalhães 1993. Inertio-gravity waves in the atmosphere of Neptune. Icarus 105, 142-161.

Hinson, D. P., and G. L. Tyler 1983. Internal gravity waves in Titan's atmosphere observed by Voyager radio occultation. Icarus 54, 337-352.

Hinson, D. P., J. D. Twicken, and E. T. Karayel 1998. Jupiter's ionosphere: New results from Voyager 2 radio occultation measurements. J. Geophys. Res. 103, 9505-9520.

Hubbard, W. B., D. M. Hunten, H. J. Reitsema, N. Brosch, Y. Nevo, E. Carreira, F. Rossi, and L. W. Wasserman 1990. Results for Titan's atmosphere from its occultation of 28 Sagittarii. Nature 343, 353-355.
Hubbard, W. B., E. Lellouch, B. Sicardy, A. Brahic, F. Vilas, P. Bouchet, and C. Perrier 1988. Structure of scintillations in Neptune's occultation shadow. Astrophys. J. 325, 490-502.

Hubbard, W. B., C. C. Porco, D. M. Hunten, G. H. Rieke, M. J. Rieke, D. W. McCarthy, V. Haemmerle, R. Clark, E. P. Turtle, J. Haller, B. McLeod, L. A. Lebofsky, R. Marcialis, J. Holberg, R. Landau, L. Carrasco, J. Elias, M. W. Buie, D. Jewitt, S. E. Persson, T. Boroson, S. West, and D. J. Mink 1993a. The occultation of $28 \mathrm{Sgr}$ by Saturn: Saturn pole position and astrometry. Icarus 103, 215-234.

Hubbard, W. B., B. Sicardy, R. Miles, A. J. Hollis, R. W. Forrest, I. K. M. Nicolson, G. Appleby, W. Beisker, C. Bittner, H.-J. Bode, M. Bruns, H. Denzau, N. Nezel, E. Riedel, H. Struckmann, J.-E. Arlot, F. Roques, F. Sévre, W. Thuillot, M. Hoffmann, E. H. Geyer, C. Buil, F. Colas, J. Lecacheux, A. Klotz, E. Thouvenot, J.-L. Vidal, E. Carreira, F. Rossi, C. Blanco, S. Cristaldi, Y. Nevo, H. J. Reitsema, N. Brosch, K. Cernis, K. Zdanavicius, L. H. Wasserman, D. M. Hunten, D. Gautier, E. Lellouch, R. V. Yelle, B. Rizk, F. M. Flasar, C. C. Porco, D. Toublanc, and G. Corugedo 1993b. The occultation of $28 \mathrm{Sgr}$ by Titan. Astron. Astrophys. 269, 541-563.

Lellouch, E., D. M. Hunten, G. Kockarts, and A. Coustenis 1990. Titan's thermosphere profile. Icarus $\mathbf{8 3}, 308-324$.

Lindal, G. F., G. E. Wood, H. B. Hotz, D. N. Sweetnam, V. R. Eshelman, and G. L. Tyler 1983. The atmosphere of Titan: An analysis of the Voyager 1 radio occultation measurements. Icarus $\mathbf{5 3}, 348-363$.

Narayan, R., and W. B. Hubbard 1988. Theory of anisotropic refractive scintillation: Application to stellar occultation by Neptune. Astrophys. J. 325, 503-518.

Roques, F., B. Sicardy, R. G. French, W. B. Hubbard, A. Barucci, P. Bouchet, A. Brahic, J.-A. Gehrels, T. Gehrels, I. Grenier, T. Lebertre, J. Lecacheux, J.-P. Maillard, R. A. McLaren, C. Perrier, F. Vilas, and M. D. Waterworth 1994. Neptune's upper atmosphere, 1983-1990: Ground-based stellar occultation observations. III. Temperature profiles. Astron. Astrophys. 288, 9851011 .

Sicardy, B., A. Brahic, C. Ferrari, D. Gautier, J. Lecacheux, E. Lellouch, F. Roques, J.-E. Arlot, F. Colas, W. Thuillot, F. Sèvre, J.-L. Vidal, C. Blanco, S. Cristaldi, C. Buil, E. Klotz, and E. Thouvenot 1990. Probing Titan's atmosphere by stellar occultation. Nature 343, 350-353.

Smith, S. A., D. C. Fritts, and T. E. VanZandt 1987. Evidence for a saturated spectrum of atmospheric gravity waves. J. Atmos. Sci. 44, 14041410 .

Strobel, D. F., and B. Sicardy 1997. Gravity Waves and Wind Shear Models, ESA-SP 1177, pp. 299-311.

Théodore, B., E. Lellouch, E. Chassefière, and A. Hauchecorne 1993. Solstitial temperature inversions in the martian middle atmosphere: Observational clues and 2-D modeling. Icarus 105, 512-528.

Vapillon, L., M. Combes, and J. Lecacheux 1973. The $\beta$ Scorpii occultation by Jupiter. II. The temperature and density profiles of the jovian upper atmosphere. Astron. Astrophys. 29, 135-149.

Washburn, E. W. 1930. International Critical Tables of Numerical Data: Physics, Chemistry and Technology, Vol. 7, p. 11. McGraw-Hill, New York.

Yelle, R. V. 1991. Non-LTE models of Titan atmosphere. Astrophys. J. 383, 380-400.

Zhu, X. 1994. A new theory of the saturated gravity wave spectrum for the middle atmosphere. J. Atmos. Sci. 51, 3615-3626. 